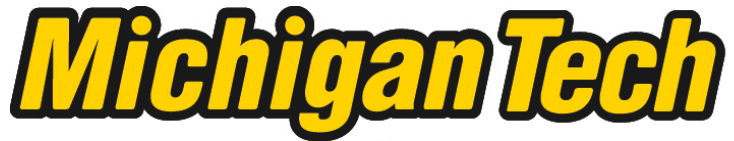 \\ Michigan Technological University Create the Future Digital Commons @ Michigan Tech
}

Dissertations, Master's Theses and Master's Reports - Open

Dissertations, Master's Theses and Master's

Reports

2013

Benson's Theorem for Partial Geometries

Ellen J. Kamischke

Michigan Technological University

Follow this and additional works at: https://digitalcommons.mtu.edu/etds

Part of the Mathematics Commons

Copyright 2013 Ellen J. Kamischke

\section{Recommended Citation}

Kamischke, Ellen J., "Benson's Theorem for Partial Geometries", Master's Thesis, Michigan Technological University, 2013.

https://doi.org/10.37099/mtu.dc.etds/690

Follow this and additional works at: https://digitalcommons.mtu.edu/etds

Part of the Mathematics Commons 


\title{
BENSON'S THEOREM FOR PARTIAL GEOMETRIES
}

\author{
By
}

Ellen Kamischke

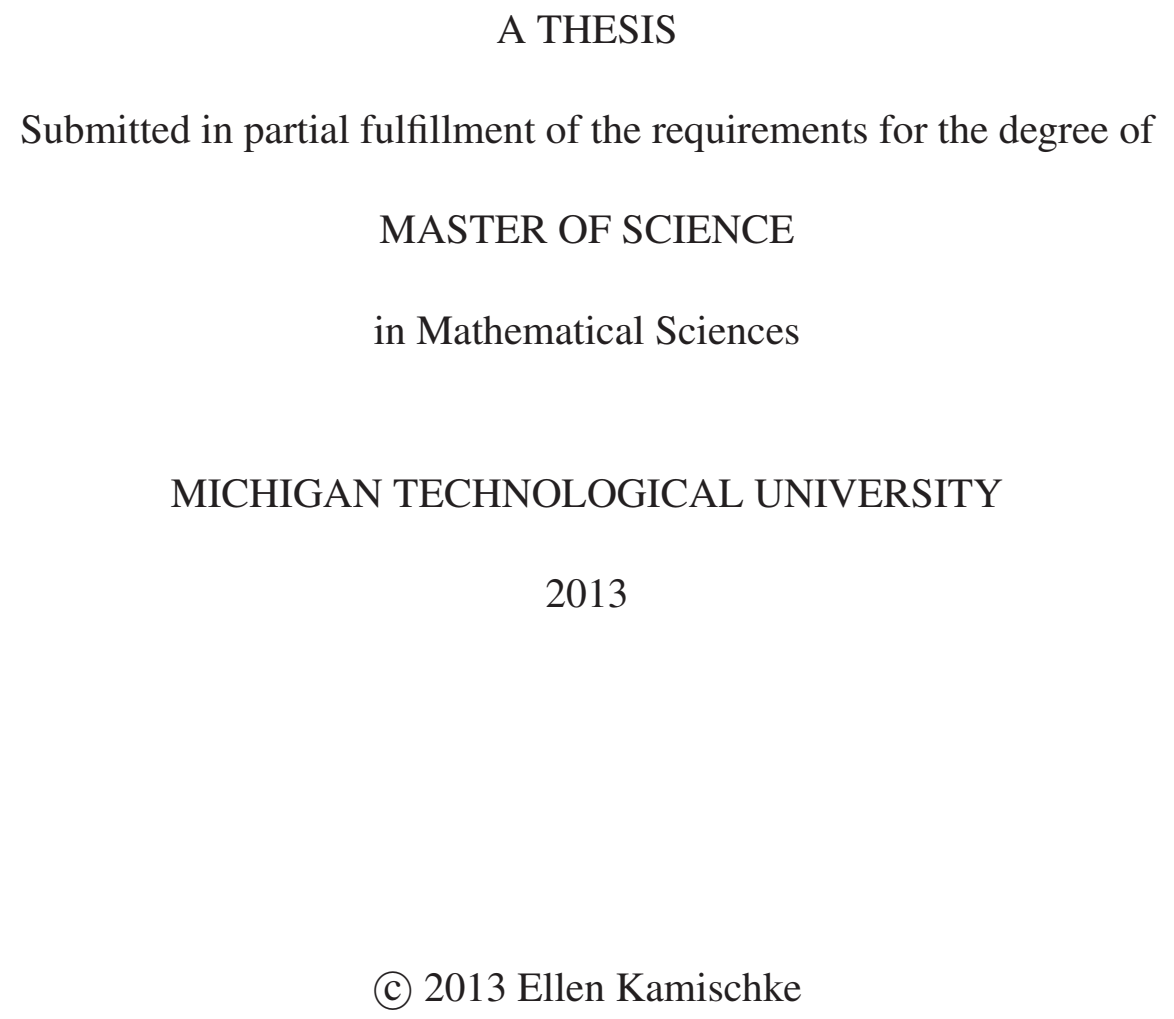



This thesis has been approved in partial fulfillment of the requirements for the Degree of MASTER OF SCIENCE in Mathematical Sciences.

Department of Mathematical Sciences

Thesis Advisor: Dr. Stefaan DeWinter

Committee Member: Dr. Melissa Keranen

Committee Member: Dr. Laura Brown

Committee Member: Dr. Rebecca Schmitz

Department Chair: Dr. Mark Gockenbach 



\section{Dedication}

For my father

Dr. O. Donald Meaders, EdD (1923 - 2012)

Teacher, scholar, friend to all 



\section{Contents}

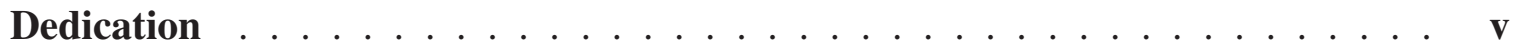

List of Figures $\ldots \ldots \ldots \ldots \ldots \ldots \ldots \ldots \ldots$

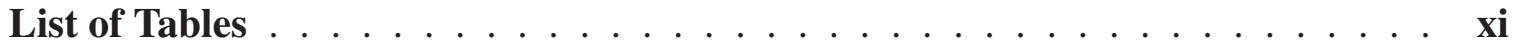

Acknowledgments ...................... xiii

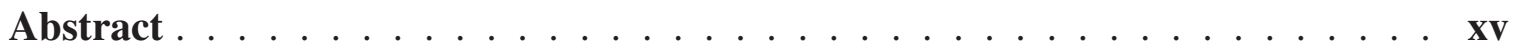

1 Introduction ........................ 1

1.1 Partial Geometries and Strongly Regular Graphs . . . . . . . . . . . . . . . 4

1.2 Properties of the adjacency matrix . . . . . . . . . . . 13

2 Benson's theorem for special cases . . . . . . . . . . . . . . 19

2.1 Automorphisms of prime order . . . . . . . . . . . . 27

2.2 Sharply transitive abelian group of automorphisms . . . . . . . . . . . . 29

3 Maximal arcs and their generalizations $\ldots \ldots \ldots$

3.1 Maximal $\operatorname{arcs} \ldots \ldots \ldots \ldots \ldots \ldots \ldots$ 
3.1.1 Construction of the partial geometry $T_{2}^{*}(\mathcal{K}) \ldots \ldots 33$

3.1.2 Applying Benson's Theorem . . . . . . . . . . . . . . 37

3.2 Generalized maximal $\operatorname{arcs} \ldots \ldots \ldots \ldots$

3.2.1 Construction of the partial geometry $\mathcal{S}(\mathcal{M}) \ldots \ldots 38$

3.2.2 Applying Benson's Theorem . . . . . . . . . . . . . . . . 40

4 Partial geometries with an abelian Singer group $\ldots \ldots \ldots$

4.1 Classifying partial geometries based on stabilizer size . . . . . . . . . 54

4.1.1 Possible parameters for rigid type geometries . . . . . . . . 55

4.2 Observations on a hypothetical geometry . . . . . . . . . . . 62

5 A Benson type theorem for strongly regular graphs . . . . . . . . . . . . . 69

5.1 Eigenvalues for a strongly regular graph . . . . . . . . . . . 70

5.2 The generalized theorem . . . . . . . . . . . . . 72

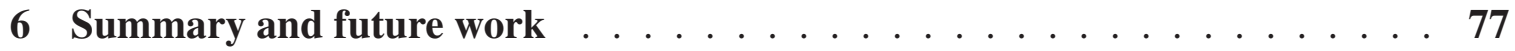

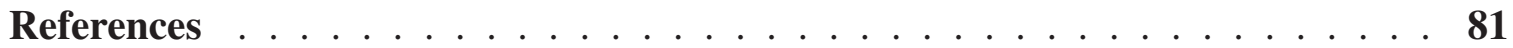

A Partial geometries and strongly regular graphs - an enrichment unit for high

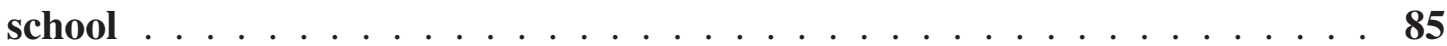




\section{List of Figures}

1.1 The visual representation of a geometry with 6 points and 4 lines and its accompanying graph ........................ 3

1.2 The incidence matrix for the geometry and the adjacency matrix for the graph. 3

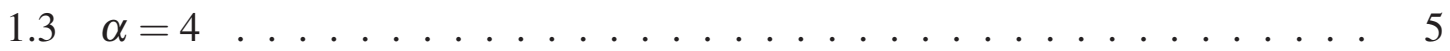

1.4 Some simple partial geometries. . . . . . . . . . . . . . . . 5

1.5 The generalized quadrangle and its point graph. . . . . . . . . . 6

1.6 An array, its geometry and the associated point graph. . . . . . . . . . . 7

1.7 Examples of strongly regular graphs and partial geometries. . . . . . . . . 7

1.8 Determining the value of $k \ldots \ldots \ldots \ldots$

1.9 Determining the value of $\mu \ldots \ldots \ldots \ldots$

1.10 Determining the value of $\lambda \ldots \ldots \ldots \ldots$

$1.11 p_{1}: s(t+1) \quad p_{2}: t(s+1-\alpha)$ for each $p_{1} \ldots \ldots 11$

$1.12 p_{2}: \mathcal{P}_{2} \quad p_{1}: \alpha(t+1)$ for each $p_{2} \ldots \ldots \ldots 12$

1.13 A geometry, its incidence matrix and the adjacency matrix for the graph. . . 14

1.14 The product of an incidence matrix and its transpose is symmetric. . . . . . 14

2.1 An automorphism of a graph and the permutation matrix. . . . . . . . 20 
3.1 The Fano plane and its incidence matrix. . . . . . . . . . . . . . . 34

3.2 Determining an upper bound for $k \ldots \ldots \ldots$. . . . . . . . . 35

3.3 The points in $\mathcal{K}$ are in groups of size $d$ in a maximal arc. . . . . . . . 35

3.4 Determining the parameters for $T_{2}^{*}(\mathcal{K}) \ldots \ldots \ldots \ldots$

3.5 The relationship between the spaces. . . . . . . . . . . . . . 39

4.1 The lines through $p_{0}$ are in groups of size $s+1 \ldots \ldots$. . . . . 56

4.2 The $\operatorname{set} D \ldots \ldots \ldots \ldots \ldots$

4.3 The action of $g_{j}^{-1}$ and then $g_{i}^{-1} \ldots \ldots \ldots \ldots$

4.4 The action of $g_{i}$ on inverses. . . . . . . . . . . . . 64

4.5 The action of $g^{-1}$ The action of $g . \ldots \ldots 5$

$5.1 p g(2,2,2)$ and its point graph . . . . . . . . . . . 72 


\section{List of Tables}

2.1 Divisibility conditions for a partial geometry when acted on by a sharply transitive abelian group of automorphisms. . . . . . . . . . . 30

3.1 sets of values for $n, m$ and $k \ldots \ldots \ldots \ldots$

4.1 Values of $s, t, \alpha$ for potential partial geometries. . . . . . . . 58

4.1 Values of $s, t, \alpha$ for potential partial geometries. . . . . . . . . 78 



\section{Acknowledgments}

The journey to this point in time has been a long one. The time from when the idea of returning to school first began to take shape in my mind until the culmination of this degree has been over ten years. The first seven of which were spent in wondering if it was really possible for me to actually do this crazy thing after more than thirty years away from serious study of mathematics. Once the decision was made to give this a try, however, I have been blessed with support and encouragement from many fronts.

The professors under whom I studied challenged me, and as a result I now see more and yet know that there is so much more out there to learn. I offer them my sincere thanks for the opportunity to learn under your direction. It was an honor and pleasure to be your student. I am also grateful to my fellow graduate students who struggled with me, helped me, and became my good friends. I wish you each the best as you continue on your own journeys. I cannot thank my advisor Dr. Stefaan DeWinter enough. His kindness and willingness to take me on as a student is appreciated beyond words. I learned much under his guidance and will be forever grateful for the chance to work with him.

I must also thank my friends Dr. Allan Struthers and Dr. Rebecca Schmitz for their support and the confidence they had in me that far exceeded what I had in myself.

Finally, I cannot thank my husband, Eric enough for all he has done to help me along this path. His love, encouragement, and support in so many different ways made this journey possible. 



\section{Abstract}

In 1970 Clark Benson published a theorem in the Journal of Algebra stating a congruence for generalized quadrangles. Since then this theorem has been expanded to other specific geometries. In this thesis the theorem for partial geometries is extended to develop new divisibility conditions for the existence of a partial geometry in Chapter 2. Then in Chapter 3 the theorem is applied to higher dimensional arcs resulting in parameter restrictions on geometries derived from these structures. In Chapter 4 we look at extending previous work with partial geometries with $\alpha=2$ to uncover potential partial geometries with higher values of $\alpha$. Finally the theorem is extended to strongly regular graphs in Chapter 5. In addition we obtain expressions for the multiplicities of the eigenvalues of matrices related to the adjacency matrices of these graphs. Finally, a four lesson high school level enrichment unit is included to provide students at this level with an introduction to partial geometries, strongly regular graphs, and an opportunity to develop proof skills in this new context. 



\section{Chapter 1}

\section{Introduction}

In the most general sense, geometry is the branch of mathematics dealing with properties and relationships between points, lines, and figures in space according to a specific set of assumptions. In school, the geometry studied is generally that of Euclid, where there are infinitely many points on each line, and infinitely many lines. This geometry rests on five assumptions or postulates.

- Any two points define a line segment.

- Any line segment can be extended indefinitely to be a line.

- Given any line segment a circle can be constructed having the segment as its radius and one endpoint as its center.

- All right angles are congruent. 
- If two lines intersect a third so that the sum of the interior angles on one side is less than two right angles, then the original two lines intersect each other on that side.

However, it is possible to change the basic assumptions, and limit the system to a finite number of points and lines. Geometries consisting of sets of objects referred to as points and lines along with an incidence relation between them are called point line geometries. It is sometimes possible to create a visual representation of a point line geometry showing all of the points and lines in their proper relationship to each other. However as the number of points increases, such a representation becomes increasingly complex. Another visualization tool that may be helpful is a point graph. In this context a graph is a collection of vertices and edges. In a point graph associated with a geometry each vertex represents a point in the geometry. The edges of the graph indicate collinearity. That is, the edges of the graph connect points that are incident to the same line, or collinear. When there are only two points on each line, the point graph and the visual representation of the geometry are identical. However, when there are more points on each line, the graph and visual representation are not the same. Figure 1.1 shows the visual representation of a geometry on the left and its associated point graph on the right. Note that each line in the geometry becomes a triangle in the graph.

The relationships in the graph can be summarized in an adjacency matrix. This is an $n \times n$ matrix $A$, where $a_{i j}=1$ if points $i$ and $j$ are collinear and 0 otherwise. Closely related to this is an $n \times m$ incidence matrix, $D$, where $a_{i j}=1$ if the $i^{t h}$ point is incident with the $j^{t h}$ 

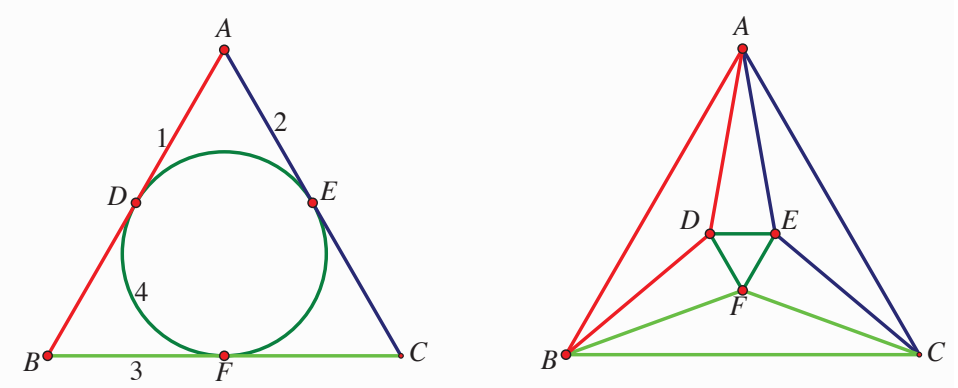

Figure 1.1: The visual representation of a geometry with 6 points and 4 lines and its accompanying graph

line. Using these matrices allows us to make use of linear algebra techniques to further explore the geometry. The incidence matrix and adjacency matrix for the geometry and point graph in Figure 1.1 are shown in Figure 1.2.

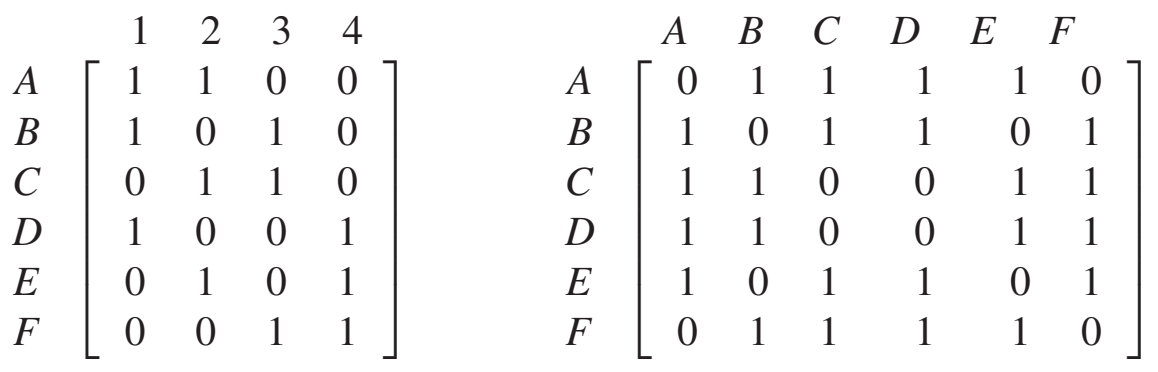

Figure 1.2: The incidence matrix for the geometry and the adjacency matrix for the graph.

Another technique for studying the structure of a particular geometry is to examine the actions of automorphisms on the points of the geometry. In the context of a geometry, an automorphism is a mapping that permutes the points and lines while preserving incidence. In general, automorphisms may permute all of the points or leave some points fixed. 


\subsection{Partial Geometries and Strongly Regular Graphs}

Partial geometries were introduced by R.C. Bose in a paper published in the Pacific Journal of Mathematics in 1963[4]. In this paper he not only defined the concept and gave some examples, but also related them to strongly regular graphs and partially balanced designs. In this thesis we will look at the parameters defining a partial geometry and present some conditions for their existence. Partial geometries are a type of point line geometry, a triple $(P, L, I)$, where $P$ and $L$ are disjoint sets referred to as points and lines, respectively, and $I$ is an incidence relation indicating when a point is on, or incident to, a line. There are four axioms that define a partial geometry:

- Any two points are incident with at most one line.

The remaining three axioms define the three parameters, $s, t$, and $\alpha$, that specify the relationships between the numbers of points and lines. The geometry is then referred to as a $p g(s, t, \alpha)$.

- There are $s+1$ points on each line.

- There are $t+1$ lines incident with every point.

- Given a point, $x$, and a line, $L$, which are not incident, there are $\alpha$ lines incident with $x$ that are also incident with points on $L$. 


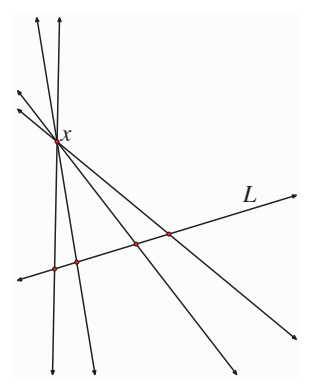

Figure 1.3: $\alpha=4$

Examples of partial geometries can be found in some simple shapes. Consider the following simple examples:
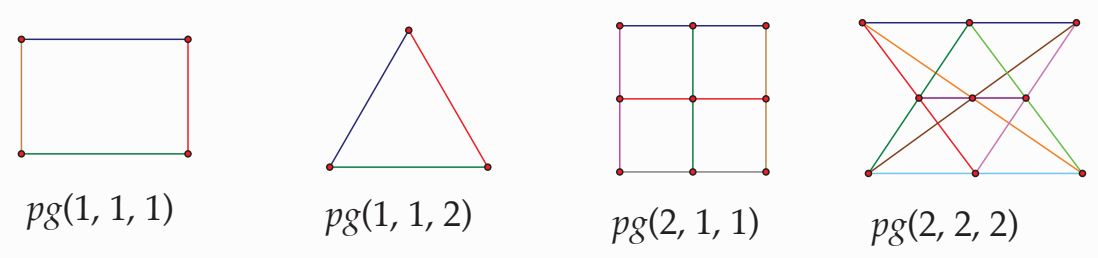

Figure 1.4: Some simple partial geometries.

When the parameters become larger, the visual representation of the geometry often becomes more complex. In some cases "lines" must be drawn as curves in order to satisfy the parameters. A classic example of this is a $p g(2,2,1)$ which is called a generalized quadrangle. It is shown in Figure 1.5 along with its accompanying point graph.

In this geometry, each of the colored arcs is a line. There are a total of 15 points and 15 lines in this partial geometry. In the graph, each geometry line becomes a triangle and there are 15 of these colored triangles in the graph. In general, partial geometries with $\alpha=1$ are 


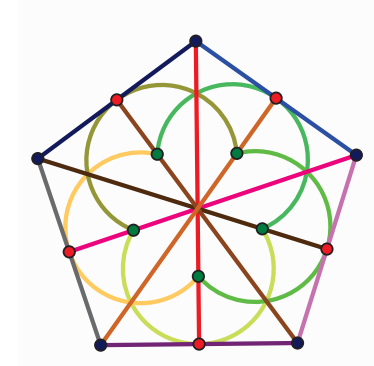

$p g(2,2,1)$

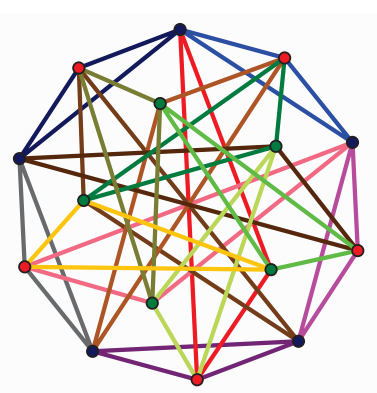

$\operatorname{srg}(15,6,1,3)$

Figure 1.5: The generalized quadrangle and its point graph.

called generalized quadrangles. This generalized quadrangle is the first of the examples presented that is classified as a proper partial geometry. That is, a partial geometry with $\alpha<\min (s, t)$. The latter portions of this paper will deal with proper partial geometries, but first we present another example of a non-proper partial geometry.

An $n \times n$ array may be filled as follows. The main diagonal is empty. The upper triangular portion is filled with consecutive integers. The lower triangular portion is then the reflection of the upper across the empty diagonal. Each row of the array is considered a line, while the integers are considered as points. Thus each line has $n-1$ points, and each point occurs on exactly 2 lines. Choosing any point and a line that does not contain it, there are always two points that are collinear with the chosen point. For example, in the array below, 7 is not on the first line, but 7 and 1 are collinear as are 7 and 4 . Geometries created in this fashion have parameters $(n-1,1,2)$ (see e.g. [4]). A visual representation of this geometry and the associated point graph are also shown for comparison with the array.

Creating a visual representation of an entire partial geometry becomes more difficult as the parameters increase. However, the point graph of a partial geometry is always a strongly 

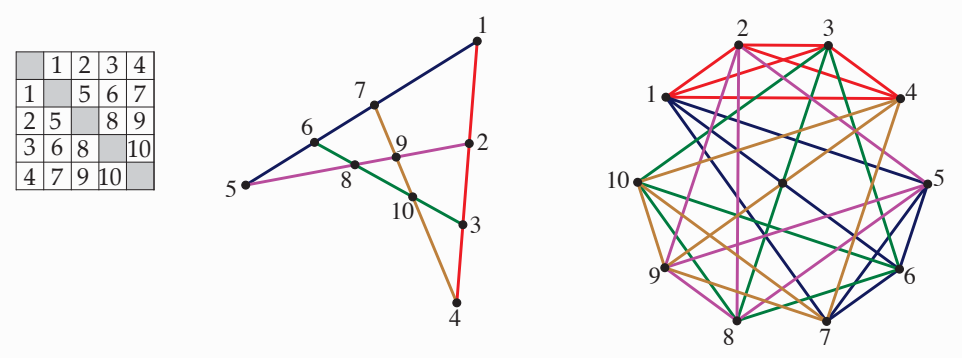

Figure 1.6: An array, its geometry and the associated point graph.

regular graph. Such a graph is described by four parameters: $v, k, \lambda$, and $\mu$ and is denoted as $\operatorname{srg}(v, k, \lambda, \mu)$. The parameter $v$ is the number of vertices in the graph, while $k$ is the degree of each vertex, or the valency of the graph. The parameter $\lambda$ gives the number of vertices that are jointly adjacent to any two adjacent vertices. Finally, $\mu$ is the number of vertices that are jointly adjacent to any two nonadjacent vertices.

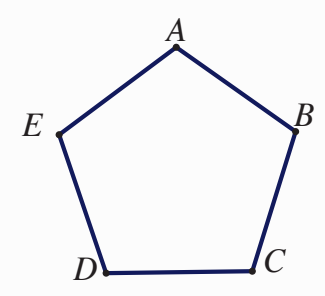

$\operatorname{srg}(5,2,0,1)$

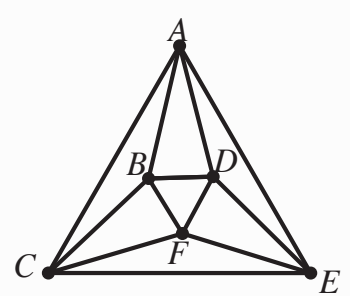

$\operatorname{srg}(6,4,2,4)$

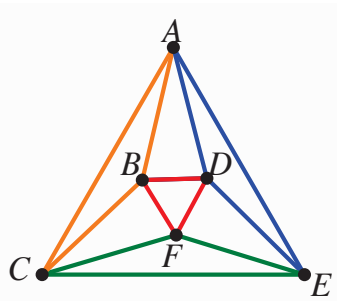

$p g(2,1,2)$

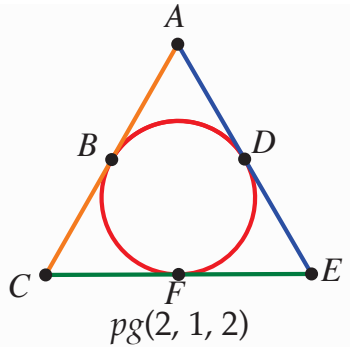

$p g(2,1,2)$

Figure 1.7: Examples of strongly regular graphs and partial geometries.

The simple pentagon in Figure 1.7 is a strongly regular graph, but cannot be the visual representation of a partial geometry. Interpreting it as a geometry there are two points on each line and two lines incident with each point, but the $\alpha$ condition is not met and so this is not a partial geometry. Consider point $A$ and line $D C$. Neither point $D$ or $C$ is collinear with $A$. But if we consider point $A$ with line $B C$, there is one point, namely $B$, that is collinear 
with $A$. The next graph above is called an octahedral graph. It is clearly a strongly regular graph. There is more than one way to interpret this figure as a representation of a geometry. If we consider the edges of the graph as lines, then there are two points on each line, four lines through each point, but no constant value of $\alpha$. Comparing point $A$ with lines $B C$ and $D F$ makes this clear. However, if we instead consider each of the colored triangles as our lines, then each line has three points and each point is on two lines. Now there is a constant $\alpha$ value of two. Point $\mathrm{A}$ is not on line $C E F$, but it is on lines $A B C$ and $A D E$. So this figure may also be interpreted as a visual representation of $p g(2,1,2)$. Another visual representation of this same geometry is shown on the far right. Here each line is indicated by a unique color.

It is easy to verify that the point graphs of each of the partial geometries presented thus far is strongly regular. In fact, many strongly regular graphs are known, but only a select number of these are point graphs of partial geometries.

The relationship described in the following theorem can help to determine which, if any, partial geometry may be associated with the strongly regular graph. This relationship is frequently stated, but generally offered without proof [5] which is why we present a detailed proof here.

Theorem 1 The point graph of a pg $(s, t, \alpha)$ is a

$$
\operatorname{srg}\left((s+1)\left(\frac{s t+\alpha}{\alpha}\right), s(t+1), s-1+t(\alpha-1), \alpha(t+1)\right) .
$$


Proof. It is simplest to prove these relationships for a $\operatorname{srg}(v, k, \lambda, \mu)$ beginning with the expression for $k$, followed by $\mu$, then $\lambda$, and finally $v$. Throughout the proof let $p_{1}$ be a point collinear with a chosen point $p$ and $p_{2}$ be a point noncollinear with $p$. Also let $\mathcal{P}_{1}$ be the number of points of type $p_{1}$ for any given point $p$. Likewise, let $\mathcal{P}_{2}$ be the number of points of type $p_{2}$ for any given point $p$.

- The number, $k$, of vertices adjacent to any given vertex. The number of vertices

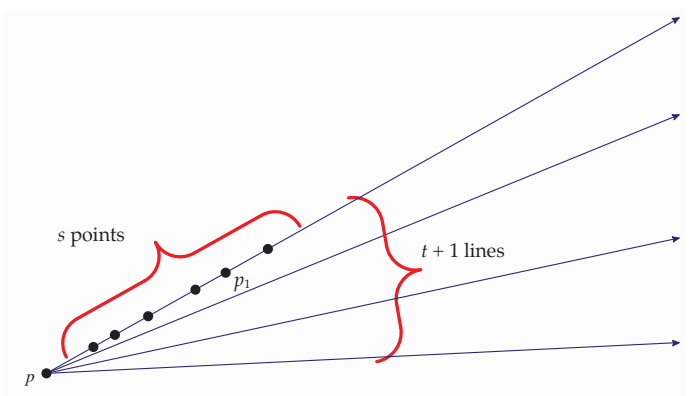

Figure 1.8: Determining the value of $k$.

adjacent to a given vertex is the number of points collinear with a given point $p$. There are $t+1$ lines incident with each point and $s$ points on each line that are collinear with $p$. So there are $s(t+1)$ points collinear with a given point. Hence there are $s(t+1)$ vertices adjacent to each vertex.

- The number, $\mu$, of vertices commonly adjacent to two nonadjacent vertices. The number of vertices commonly adjacent to two nonadjacent vertices is the same as the number of points collinear with two noncollinear points. Let $p$ and $p_{2}$ be two noncollinear points. There are $t+1$ lines through $p_{2}$. None of them are incident with $p$. On each one there are $\alpha$ points that are collinear with $p$. So there are a total of 


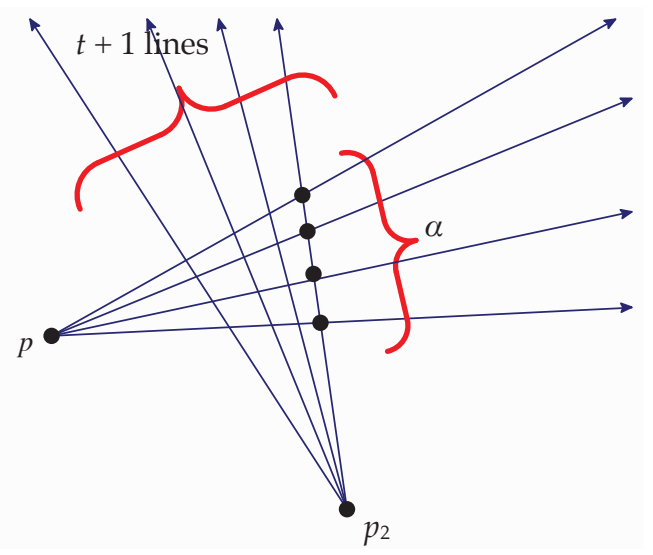

Figure 1.9: Determining the value of $\mu$.

$\alpha(t+1)$ points that are collinear with $p$ and $p_{2}$ and thus there are $\alpha(t+1)$ vertices that are commonly adjacent to two nonadjacent vertices.

- The number, $\lambda$, of vertices commonly adjacent to 2 adjacent vertices. The number

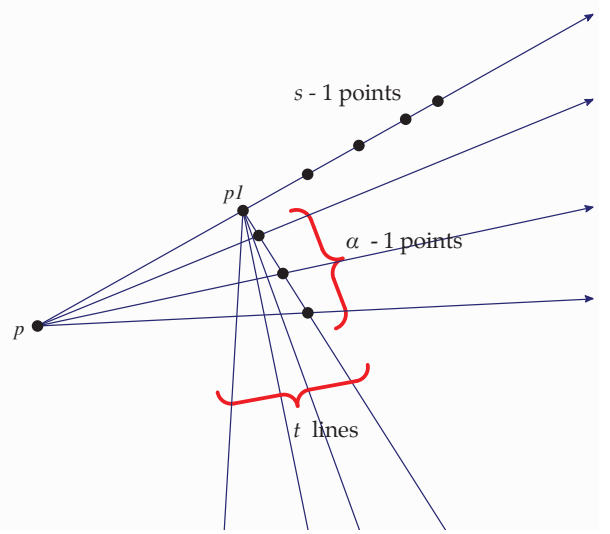

Figure 1.10: Determining the value of $\lambda$.

of vertices commonly adjacent to 2 adjacent vertices is the same as the number of points that are collinear with two given collinear points $p$ and $p_{1}$. Since each line contains $s+1$ points, there are $s-1$ more points on the line containing $p$ and $p_{1}$ that 
are collinear to both.

There are $t$ lines through $p_{1}$, omitting the line containing $p$ and $p_{1}$. Each of these lines contains $\alpha$ points that are collinear with $p$. However $p_{1}$ is on each of these lines. So there are $\alpha-1$ points on each of the $t$ lines that are collinear to the two given collinear points. This makes a total of $s-1+t(\alpha-1)$ vertices commonly adjacent to 2 adjacent vertices.

- The number, $v$, of vertices.

The vertices of the point graph are the points of the geometry. Selecting any point $p$, the remaining points are of two types; points, $p_{1}$, that are collinear with $p$, and points, $p_{2}$, that are not collinear with $p$. We will count the pairs $\left(p_{1}, p_{2}\right)$ where $p_{1}$ is collinear with $p_{2}$ in two ways.

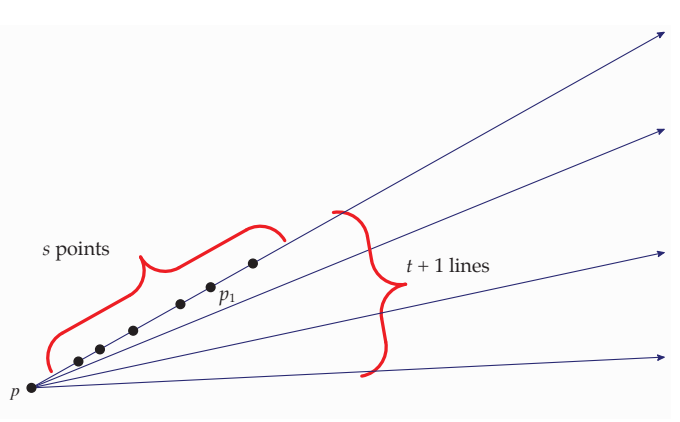

Figure 1.11: $p_{1}: s(t+1)$

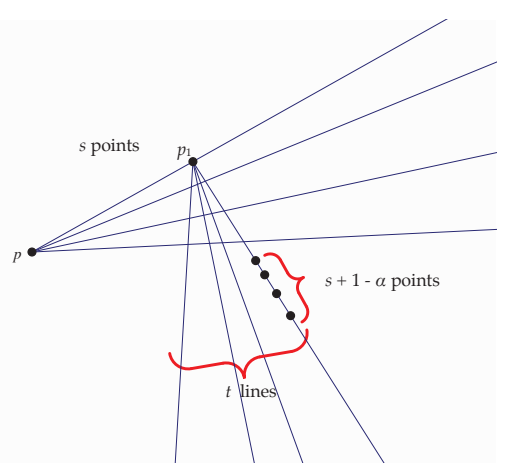

$p_{2}: t(s+1-\alpha)$ for each $p_{1}$.

First, from the first part of the proof we know there are there are $s(t+1)$ possible points $p_{1}$. Then for each of these $p_{1}$ points there are $t$ lines that are not incident with p. Each of these lines has $\alpha$ points that are collinear with $p$ and $s+1-\alpha$ points 
that are not. These $s+1-\alpha$ points are all possible points $p_{2}$. So there are $s+1-\alpha$ points, $p_{2}$, on each of these $t$ lines. Because two lines intersect in at most one point, none of these points is double counted. Thus for each $p_{1}$ there are $t(s+1-\alpha)$ points $p_{2}$. The total number of pairs of points $\left(p_{1}, p_{2}\right)$ is $s(t+1) \cdot t(s+1-\alpha)$.

For the second counting method we know from the second part of the proof that for any point $p_{2}$ there are $\alpha(t+1)$ choices for $p_{1}$. Thus the total number of pairs of points $\left(p_{1}, p_{2}\right)$ is $\alpha(t+1) \mathcal{P}_{2}$, where $\mathcal{P}_{2}$ is the number of points of type $p_{2}$. Equating

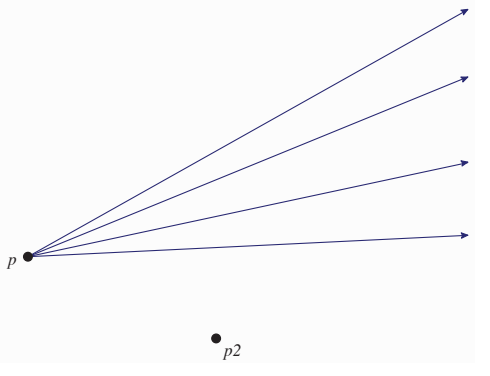

Figure 1.12: $p_{2}: \mathcal{P}_{2}$

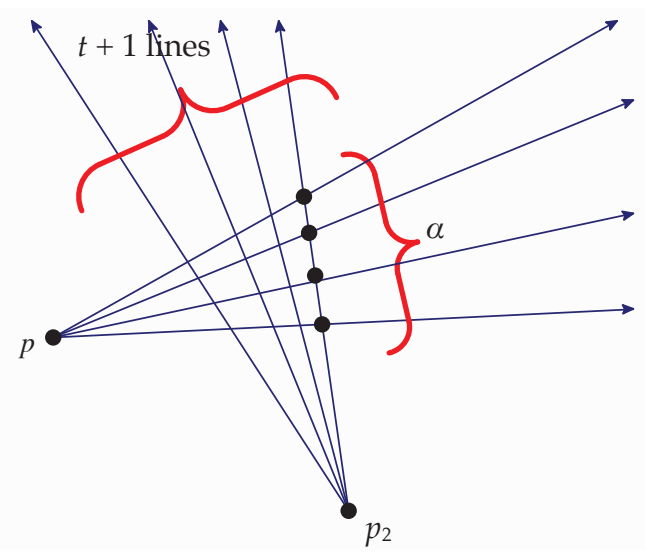

$p_{1}: \alpha(t+1)$ for each $p_{2}$.

the two counts and solving for $\mathcal{P}_{2}$ gives the following:

$$
\begin{aligned}
& s t(t+1)(s+1-\alpha)=\mathcal{P}_{2} \alpha(t+1) \\
& \mathcal{P}_{2}=\frac{s t(s+1-\alpha)}{\alpha}
\end{aligned}
$$

The total number of points is now $1+\mathcal{P}_{1}+\mathcal{P}_{2}=1+s(t+1)+\frac{s t(s+1-\alpha)}{\alpha}$ 
Simplifying, we get

$$
\begin{aligned}
1+s(t+1)+\frac{s t(s+1-\alpha)}{\alpha}= & \frac{\alpha+\alpha s t+\alpha s+s t(s+1)-\alpha s t}{\alpha} \\
= & \frac{\alpha(s+1)+s t(s+1)}{\alpha} \\
& =(s+1) \frac{(\alpha+s t)}{\alpha}
\end{aligned}
$$

This proves the point graph of a $p g(s, t, \alpha)$ is strongly regular with parameters $\operatorname{srg}\left((s+1)\left(\frac{s t+\alpha}{\alpha}\right), s(t+1), s-1+t(\alpha-1), \alpha(t+1)\right)$.

This relationship between strongly regular graphs and partial geometries enables us to make use of information about the associated graph to further investigate the parameters of the partial geometry. We will need some results concerning the adjacency matrix and incidence matrix in the next chapter.

\subsection{Properties of the adjacency matrix}

For any graph, $G$ of order $n$, the adjacency matrix is an $n \times n$ square matrix where each row and column corresponds to a vertex of $G$. Let the vertices be denoted as $v_{1}, v_{2}, \ldots, v_{n}$. The entries, $a_{i j}$, of this matrix are defined as $a_{i j}=\left\{\begin{array}{ll}1 & \text { if } v_{i} \text { is adjacent to } v_{j} \\ 0 & \text { otherwise }\end{array}\right.$. An incidence matrix for a finite point line geometry is an $n \times m$ matrix where $n$ is the number of points and $m$ is the number of lines. Let the points be denoted by $x_{1}, x_{2}, \ldots, x_{n}$ and the lines by $l_{1}, l_{2}, \ldots, l_{m}$. Each entry, $a_{i j}$ is defined as $a_{i j}=$ 
$\left\{\begin{array}{ll}1 & \text { if } x_{i} \text { is incident to } l_{j} \\ 0 & \text { otherwise }\end{array}\right.$. So the number of 1 's in a column is the number of points incident to a line. As an example, consider the partial geometry $p g(1,1,1)$, its incidence matrix, and the adjacency matrix for the graph associated with this geometry.

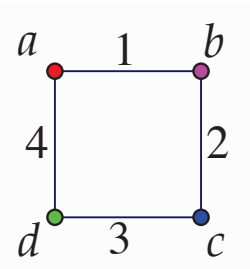

$$
\begin{aligned}
& a \\
& b \\
& c \\
& d
\end{aligned}\left[\begin{array}{llll}
a & b & c & d \\
0 & 1 & 0 & 1 \\
1 & 0 & 1 & 0 \\
0 & 1 & 0 & 1 \\
1 & 0 & 1 & 0
\end{array}\right] \quad \begin{aligned}
& a \\
& b \\
& c \\
& d
\end{aligned}\left[\begin{array}{llll}
1 & 2 & 3 & 4 \\
1 & 0 & 0 & 1 \\
1 & 1 & 0 & 0 \\
0 & 1 & 1 & 0 \\
0 & 0 & 1 & 1
\end{array}\right]
$$

Figure 1.13: A geometry, its incidence matrix and the adjacency matrix for the graph.

The first row of the adjacency matrix $A$ shows that point $a$ is collinear with points $b$ and $d$. The first row of the incidence matrix $D$ shows that point $a$ is incident with lines 1 and 4 . Note that while an adjacency matrix is always square, an incidence matrix is only square if the number of points is equal to the number of lines. However, if the incidence matrix is multiplied by its transpose, the product is square and symmetric. Using the incidence matrix above as an example, we see the symmetric product in Figure 1.14.

$$
D D^{T}=\left[\begin{array}{llll}
1 & 0 & 0 & 1 \\
1 & 1 & 0 & 0 \\
0 & 1 & 1 & 0 \\
0 & 0 & 1 & 1
\end{array}\right]\left[\begin{array}{llll}
1 & 1 & 0 & 0 \\
0 & 1 & 1 & 0 \\
0 & 0 & 1 & 1 \\
1 & 0 & 0 & 1
\end{array}\right]=\left[\begin{array}{llll}
2 & 1 & 0 & 1 \\
1 & 2 & 1 & 0 \\
0 & 1 & 2 & 1 \\
1 & 0 & 1 & 2
\end{array}\right]
$$

Figure 1.14: The product of an incidence matrix and its transpose is symmetric. 
The entries in this resulting matrix are the dot products of the $i$ th row of $D$ and the $j$ th column of $D^{T}$. Ones in the $i$ th row of $D$ represent lines incident to the $i$ th point, while ones in the $j$ th column of $D^{T}$ represent lines that are incident to the $j$ th point. When $i \neq j$, this product will either be one if the two points are incident to the same line, or zero if they are not. The product cannot be more than one as this would mean the two points were incident to more than one line. Therefore, the off-diagonal elements of $D D^{T}$ are either one or zero and are copies of the corresponding entries in $A$. If $i=j$, the product gives the number of lines incident to a single point. The product of the incidence matrix and its transpose will be used in the proof of Theorem 4 . We define this product formally as follows: Let $D$ be an $m \times n$ incidence matrix for a partial geometry. Then $D D_{i j}^{T}=\sum_{i=1}^{m} \sum_{j=1}^{n} d_{i j} d_{j i}^{T}$. Also, $D D_{i i}^{T}=t+1, D D_{i j}^{T}=a_{i j}$ where $a_{i j}$ is the element of the adjacency matrix for the graph associated with the partial geometry. Next we consider some properties of the adjacency matrix beginning with a frequently stated equation involving the matrix and the parameters of a strongly regular graph[5].

Lemma 2 The adjacency matrix of a strongly regular graph $\operatorname{srg}(v, k, \lambda, \mu)$ satisfies the equations $A^{2}+(\mu-\lambda) A+(\mu-k) I=\mu J$ and $A J=k J$ where $I$ is the identity matrix and $J$ is the all-1-matrix.

Proof. Let $A$ be the $n \times n$ adjacency matrix for a strongly regular graph. Let $n_{i j}$ be the $i j$ th entry in the product matrix $A J$. Then because $J$ is the all-1-matrix, $n_{i j}$ is the number of vertices adjacent to vertex $i$. Because the graph is regular, this value is constant and is simply $k$. Thus $A J=k J$. 
Consider the matrix $A^{2}$. Let $a_{i j}^{(2)}$ represent the entry in row $i$ and column $j$. Because $A$ is square and symmetric, this entry is the dot product of two vectors. Thus it sums the number of vertices commonly adjacent to vertex $i$ and vertex $j$. If $i=j$ this is simply $k$. If vertex $i$ and vertex $j$ are adjacent, then $a_{i j}^{(2)}=\lambda$. If they are not adjacent, $a_{i j}^{(2)}=\mu$. This same matrix can also be created by the following steps. First multiply $\lambda A$. This replaces all of the ones in $A$ with $\lambda$ which is the value we want in the final result. Next we add $\mu J$ to replace the zeros with the desired value. However, we have added $\mu$ to everything, so we subtract $\mu A$ to remove this additional count from the entries representing adjacent vertices and return those values back to $\lambda$. Now the only thing to create is the proper values on the diagonal. To do this, we subtract $\mu I$ and add $k I$. This first zeros the diagonal and then replaces the zeros with $k$ as required. Thus the matrix $\lambda A-\mu J-\mu A-\mu I+k I=A^{2}$. Rearranging this equation we get $A^{2}+(\mu-\lambda) A+(\mu-k) I=\mu J$ as desired.

We will also need to work with the eigenvalues of the adjacency matrix.

Lemma 3 The eigenvalues of the adjacency matrix of a strongly regular graph $\operatorname{srg}(v, k, \lambda, \mu)$ are $k$ and two others, $r$ and $l$, with the property that $r+l=\mu-\lambda$ and $r l=\mu-k$

Proof. From the equation $A J=k J$ we see that $k$ is an eigenvalue for $A$.

Because $A$ is symmetric, the eigenvectors of $A$ will be orthogonal [11]. Let $v$ be an eigenvector of $A$, not associated with the eigenvalue $k$. By orthogonality, its dot product 
with any other eigenvector will be zero [11]. This means $k J v=0$. Dotting the equation $A^{2}+(\mu-\lambda) A+(\mu-k) I=k J$ on both sides with $v$ we get $A^{2} v+(\mu-\lambda) A v+(\mu-k) I v=$ $k J v=0$. If $\theta$ is the eigenvalue associated with $v$, then $A v=\theta v$. Thus the equation can be written as $\theta^{2} v+(\mu-\lambda) \theta v+(\mu-k) I v=0$ or $\left(\theta^{2}+(\mu-\lambda) \theta+(\mu-k) I\right) v=0$. So $\theta$ must satisfy the equation $\theta^{2}+(\mu-\lambda) \theta+(\mu-k)=0$. Let $r, l$ be the roots of this quadratic equation. With a leading coefficient of one, the sum of the roots is the opposite of the linear coefficient. So $r+l=\lambda-\mu$. The product of the roots is the constant term so $r l=\mu-k$

With this background established we now apply these ideas to Benson's theorem and develop new divisibility conditions for partial geometries. 



\section{Chapter 2}

\section{Benson's theorem for special cases}

In 1969 Benson proved a series of lemmas dealing with automorphisms of generalized quadrangles (partial geometries with $\alpha=1$ ) [2]. The properties of the adjacency matrix of the graph associated with the partial geometry are used in establishing these lemmas. An automorphism of a geometry is a bijection, or one-to-one and onto mapping, of points that sends lines to lines. Thus it preserves incidence. The automorphisms form a group under composition as the identity mapping is an automorphism, each automorphism has an inverse, and composition of mappings is associative. When a geometry is acted on by an automorphism, the actions on the points can be expressed as a matrix. For instance, the geometry below left becomes the one below center after a $90^{\circ}$ clockwise rotation. The matrix in Figure 2.1 is a representation of this automorphism.

In Figure 2.1 the "1" in the first row, second column of the matrix indicates that the point originally labeled $a$ is now in the position originally occupied by the point labeled $b$. This 


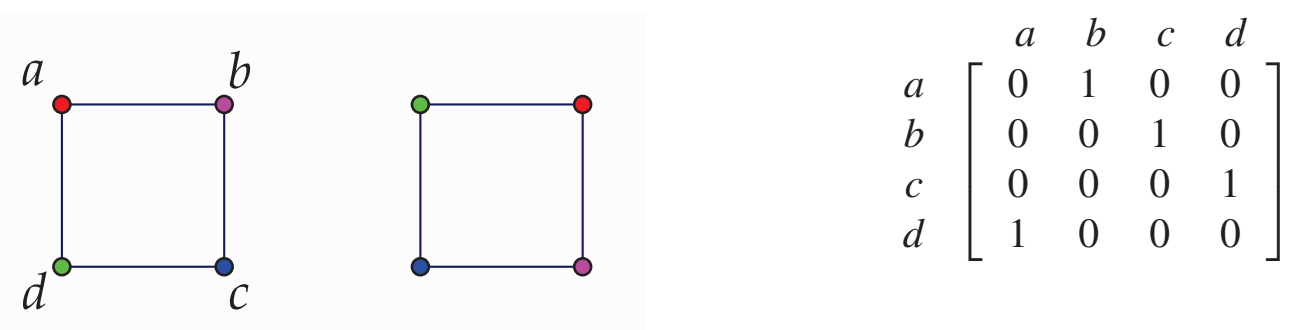

Figure 2.1: An automorphism of a graph and the permutation matrix.

matrix is an example of a permutation matrix. A permutation matrix is an $n \times n$ matrix that has exactly one 1 in each row and column and zeros elsewhere. Multiplying a matrix $M$ on the left by a permutation matrix $P$ results in interchanging the rows of $M$ according to how $P$ differs from the identity matrix. For instance the permutation matrix above results from replacing the first row of $I$ with the second row. The permutation moves point $a$ to the former location of point $b$. Similarly, the second row of $I$ has been replaced by the third row in forming $P$, and point $b$ moves to the former location of point $c$. Ones along the diagonal of $P$ thus represent points that are mapped to themselves by the automorphism. The sum of the diagonal elements of a matrix is called the trace of the matrix. The trace is also the sum of the eigenvalues of the matrix [11]. We will make use of both interpretations of the trace shortly.

One result of Benson was that if $P_{\theta}$ is the permutation matrix for the automorphism $\theta$ of a generalized quadrangle, and $M=A+(t+1) I$, then $\operatorname{trace}\left(P_{\theta} M\right)=(t+1) f+g=$ $(1+s)(1+t)+z(s+t)$ where $z \in \mathbb{Z}$. Here $f$ is the number of fixed points under the automorphism and $g$ is the number of points that are collinear with their images under $\theta$. This result has been extended for partial geometries by De Winter in 2005 [10] and for 
partial quadrangles by Temmermans in 2010 [15]. A partial quadrangle is specified by three parameters, $s, t, \mu$. Here $s$ and $t$ are the same as for a partial geometry. However, in a partial quadrangle, given a line and a point not incident with the given line, there are either no points on the line collinear with the given point or there is exactly one point on the line collinear with the given point. Thus for some point-line pairs the value of $\alpha$ is zero while for other point-line pairs, the value of $\alpha$ is one. The value of $\mu$ is a constant such that for any pair of non-collinear points, $a$ and $b$, there are exactly $\mu$ points collinear with both $a$ and $b$. An expanded version of DeWinter's proof for partial geometries is presented below as it forms the basis for the ideas that follow.

Theorem 4 Let $\mathcal{S}$ be a partial geometry $p g(s, t, \alpha)$ and let $\theta$ be any automorphism of $\mathcal{S}$. Denote by $f$ the number of fixed points of $\mathcal{S}$ under $\theta$ and by $g$ the number of points $x$ of $\mathcal{S}$ for which $x \neq x^{\theta} \sim x$, i.e. $x$ is collinear with $x^{\theta}$, where $x^{\theta}$ denotes the image of $x$ under $\theta$. Then

$$
(1+t) f+g \equiv(1+s)(1+t)(\bmod s+t-\alpha+1)
$$

Proof. Let $\mathcal{P}$ be the point set of $\mathcal{S}$, and let $A$ be the adjacency matrix of $\mathcal{S}$. Because the point graph of a partial geometry is a strongly regular graph we know that $A$ satisfies

$$
A^{2}+(\mu-\lambda) A+(\mu-k) I=\mu J \text { and } A k=k J
$$

By Lemma 3 we know that the eigenvalues of $A$ are $k, r$, and $l$, where $r+l=\lambda-\mu$ and $r l=$ $\mu-k$. In addition, for $\operatorname{pg}(s, t, \alpha)$, the corresponding strongly regular graph has parameters 
$v=(s+1) \frac{s t+\alpha}{\alpha}, k=s(t+1), \lambda=s-1+t(\alpha-1), \mu=\alpha(t+1)$. Equation (1) then becomes:

$$
\begin{gathered}
A^{2}+(\alpha(t+1)-s+1-t(\alpha-1)) A+(\alpha(t+1)-s(t+1)) I=\alpha(t+1) J \\
A^{2}+(\alpha-s+t+1) A+(t+1)(\alpha-s) I=\alpha(t+1) J
\end{gathered}
$$

Using the values from above, the first eigenvalue of $A$, that is, $k$, becomes $s(t+1)$. The remaining eigenvalues are computed as follows:

$$
\begin{aligned}
& r+l=s-1+t(\alpha-1)-\alpha(t+1)=s-\alpha-t-1 \\
& r l=\alpha(t+1)-s(t+1)=(\alpha-s)(t+1)
\end{aligned}
$$

Solving the first equation for $r$ and substituting into the second:

$$
\begin{gathered}
r=s-\alpha-t-1-l \\
(s-\alpha-t-1-l) l=(\alpha-s)(t+1) \\
0=l^{2}+(1-s+\alpha+t) l+(\alpha-s)(t+1)
\end{gathered}
$$

Solving for $l$ using the quadratic formula:

$$
\begin{aligned}
l & =\frac{s-\alpha-t-1 \pm \sqrt{(1-s+\alpha+t)^{2}-4(\alpha-s)(t+1)}}{2} \\
& =\frac{s-\alpha-t-1 \pm \sqrt{(1+s-\alpha+t)^{2}}}{2} \\
& =s-\alpha,-t-1
\end{aligned}
$$

Because $r$ and $l$ are defined symmetrically, we have without loss of generality,

$$
r=s-\alpha, l=-t-1
$$


The multiplicities of the eigenvalues are then $a_{0}=1, a_{1}, a_{2}$ where $a_{1}$ and $a_{2}$ can be determined from the following system of equations:

$$
\left\{\begin{array}{l}
k+a_{1} r+a_{2} l=0 \\
a_{1}+a_{2}=v-1
\end{array}\right.
$$

The first equation comes from the fact that the sum of the eigenvalues is the trace and the trace of $A$ is zero. The second equation comes from the fact that there are $n$ real eigenvalues for a symmetric real $n \times n$ matrix, and $n=v$.

We can replace $k, r, l$, and $v$ with their equivalent values in terms of $s, t$, and $\alpha$ to get the following:

$$
\left\{\begin{array}{l}
s(t+1)+a_{1}(s-\alpha)+a_{2}(-t-1)=0 \\
a_{1}+a_{2}=\frac{s^{2} t+s \alpha+s t}{\alpha}
\end{array}\right.
$$

Multiplying the second equation in $(2.3)$ by $(s-\alpha)$ and then subtracting the first equation from this result, we get

$$
a_{2}(s+t+1-\alpha)=\frac{(s-\alpha)\left(s^{2} t+s \alpha+s t\right)+s \alpha(t+1)}{\alpha}
$$

Expanding the numerator on the right, collecting like terms, and then factoring we get:

$$
a_{2}(s+t+1-\alpha)=\frac{s(s t+\alpha)(s+1-\alpha)}{\alpha}
$$


Finally solving for $a_{2}$ we get

$$
a_{2}=\frac{s(s t+\alpha)(s+1-\alpha)}{\alpha(s+t+1-\alpha)}
$$

Substituting this back in (2.3) and solving for $a_{1}$ we get

$$
a_{1}=\frac{s t(s+1)(t+1)}{\alpha(s+t+1-\alpha)}
$$

Let $D$ denote the incidence matrix of $S$ and define $M=D D^{T}$. As discussed earlier, the diagonal elements indicate the number of lines incident to a single point. In a partial geometry this is always $t+1$. So the diagonal elements all have this value. The off-diagonal elements are either 0 or 1 and are copies of the corresponding entries in the adjacency matrix. Therefore $M=D D^{T}=A+(t+1) I$.

Adding $(t+1) I$ to $A$ also adds $t+1$ to the eigenvalues of $A$, so the eigenvalues of $M$ are $s(t+1)+(t+1)=(s+1)(t+1), s-\alpha+(t+1),-t-1+(t+1)=0$, with the same multiplicities as before.

We now consider an automorphism of the geometry, $\theta$, its permutation matrix, $Q$, and the product $Q M$. Let $x_{i}$ represent the $i^{t h}$ point. Then $Q$ is the matrix with $q_{i j}=1$ if $x_{i}^{\theta}=x_{j}$ and 0 otherwise. That is, if the image of $x_{i}$ under $\theta$ is $x_{j}$, then there will be a 1 in the $i j$ th position of $Q$. Thus $Q$ is a permutation matrix showing how the points of the geometry are permuted by the automorphism, $\theta$. We claim $Q M=M Q$. At this point we follow the original method used by Benson and others. Later we will see an alternate method which allows 
further generalizations. To begin, consider the matrix $R$ that shows the automorphism as a permutation of the lines. The product $D R$ permutes the lines of $S$ as each entry in $D R$ is the dot product of a row of $D$ indicating the lines incident with a particular point and the column of $Q$ indicating the permutation. Thus the product works in the same way as $Q D$ only with the lines. So $Q D=D R$. Multiplying on the left by $Q^{-1}$ we get $D=Q^{-1} D R$. Then taking the transpose of this we get $D^{T}=R^{T} D^{T}\left(Q^{-1}\right)^{T}$. Now since $M=D D^{T}$, then $Q M=Q D D^{T}=D R\left(R^{T} D^{T}\left(Q^{-1}\right)^{T}\right)$. As $Q$ and $R$ and both permutation matrices each has only one 1 in each row and column. This means they are both orthogonal matrices. As such, each matrix is its own inverse and also its own transpose. Thus $Q^{T}=Q^{-1}$ and likewise, $R^{T}=R^{-1}$. So $Q M=D\left(R R^{T}\right) D^{T}\left(Q^{-1}\right)^{T}=D D^{T} Q=M Q$ as claimed. Also, if $\theta$ has order $n$, then $(Q M)^{n}=Q^{n} M^{n}=M^{n}$ because $Q^{n}$ is the identity.

Now consider the set of eigenvalues, $\delta_{i}, i=1,2, \ldots, v$, of $Q M$. Then $Q M x_{i}=\delta_{i} x_{i}$ for some corresponding eigenvector $x_{i}$. Multiplying on the left by $Q M$ gives $(Q M)^{2} x_{i}=Q M \delta_{i} x_{i}=$ $\delta_{i} Q M x_{i}=\delta_{i}^{2} x$. Continuing to multiply by $Q M$ leads to $(Q M)^{n} x_{i}=\delta_{i}^{n} x_{i}$ or $M^{n} x_{i}=\delta_{i}^{n} x$. Now suppose $\gamma_{i}, i=1,2, \ldots, v$, is the set of eigenvalues of $M$, and let $p(x)=x^{n}$. Then the eigenvalues of $p(M)=M^{n}$ are $p\left(\gamma_{i}\right)=\gamma_{i}^{n}$ [12]. So the set of eigenvalues of $M^{n}$ represented by $\delta_{i}^{n}$ is the same as the set of eigenvalues represented by $\gamma_{i}^{n}$. Thus the eigenvalues of $Q M$ are the eigenvalues of $M$ multiplied by the appropriate roots of unity.

We also know that $(Q M) J=(M Q) J=M(Q J)=M J$. This is because $Q$ is a matrix with only one nonzero entry in each row and column and multiplying this by $J$, the all one matrix simply returns $J$. Because $M J=(s+1)(t+1) J$ it follows that $(s+1)(t+1)$ is an 
eigenvalue of $Q M$ with multiplicity 1 . Also, as $M$ was defined by $M=A+(t+1) I$, the eigenvalues of $M$ and thus $Q M$, up to roots of unity, are the eigenvalues of $A$ plus $t+1$. So one of the remaining eigenvalues of $Q M$ is $-t-1+t+1=0$ with multiplicity $a_{2}$.

The remaining possible eigenvalues are obtained by multiplying $s+t-\alpha+1$ by each of the $d^{\text {th }}$ primitive roots of 1 , where $d$ is any divisor of $n$, the order of the automorphism $\theta$. Because each of the $d^{\text {th }}$ primitive roots has the same multiplicity, ${ }^{1}$ these multiplicities depend only on $d$, not on the individual primitive roots. Let $a_{d}$ represent the multiplicity of each of the eigenvalues associated with the $d^{\text {th }}$ roots. The sum of all of the $d^{\text {th }}$ primitive roots of unity is an integer, $\mu(d)$ where

$$
\mu(d)=\left\{\begin{array}{cc}
1 & d \text { is square free and has an even number of prime factors } \\
-1 & d \text { is square free and has an odd number of prime factors } \\
0 & d \text { is not square free }
\end{array}\right.
$$

The trace of a matrix is the sum of the eigenvalues, so trace $(Q M)=\sum_{d \mid n} a_{d}(s+t+\alpha-$ 1) $\mu(d)+(s+1)(t+1)$. This is clearly congruent to $(s+1)(t+1)(\bmod s+t+\alpha-1)$. However, the trace $(Q M)$ is also equal to $(t+1) f+g$ as each entry on the diagonal of $Q M$ is $t+1$ if the corresponding point is fixed and 1 if the corresponding point is mapped to a collinear point by the automorphism. To see this, recall that $M=(t+1) I+A$. So when we multiply $Q M$, if the $i^{t h}$ point is fixed, then $(Q M)_{i i}=t+1$. If the point is not fixed, then the single one in the $i^{t h}$ row of $Q$ will not match with the $t+1$ on the diagonal of $M$, but will

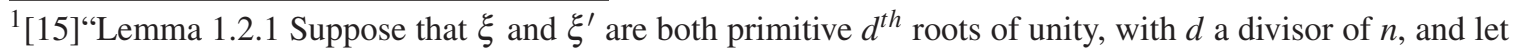
$\lambda$ be an integer eigenvalue of $M$. If at least one of $\xi \lambda$ and $\xi^{\prime} \lambda$ is an eigenvalue of $Q M$, then they both are and they have the same multiplicity." 
instead match with a one elsewhere in the $i^{t h}$ column of $M$ if and only if the the $i^{t h}$ point is being mapped to a collinear point.

Therefore, $(t+1) f+g \equiv(s+1)(t+1)(\bmod s+t+\alpha-1)$.

In her dissertation, Temmermans generalized Benson's theorem for various structures such as generalized hexagons, octagons, dodecagons, and various types of designs [2]. We now look at more specific instances of automorphisms.

\subsection{Automorphisms of prime order}

The previous proof resulted in a congruence. However, if we consider only automorphisms of prime order, we can reach additional results. Specifically, we can determine expressions for the multiplicities of the eigenvalues which will then yield new divisibility conditions on the parameters of a partial geometry.

Theorem 5 Given a partial geometry $p g(s, t, \alpha)$ acted upon by an automorphism of prime order, $n$, the matrix $Q M$ as defined in the proof of Theorem 4 has eigenvalues with multiplicities $a_{1}$ and $a_{2}$ as follows:

$$
\begin{aligned}
& a_{1}=\frac{\alpha(n-1)((t+1) f+g)+(s+1)(t+1)(s t-\alpha(n-1))}{\alpha n(s+t+1-\alpha)} \\
& a_{2}=\frac{(s+1)(t+1)(s t+\alpha)-\alpha((t+1) f+g)}{\alpha n(s+t+1-\alpha)}
\end{aligned}
$$

Proof. In the proof of Theorem 4 we obtained the equation $\sum_{d \mid n} a_{d}(s+t+1-\alpha) \mu(d)+(s+$ 1) $(t+1)=(t+1) f+g$ where $a_{d}$ is the multiplicity of the eigenvalue multiplied by the primitive $d^{\text {th }}$ root of unity, and $\mu(d)$ is the sum of the $d^{\text {th }}$ roots of unity. When $n$ is prime, 
this summation has only two terms, $a_{1}(s+t+1-\alpha)+a_{2} \mu(n)(s+t+1-\alpha)$ where $\mu(n)$ is the sum of the primitive $n^{\text {th }}$ roots of unity. But when $n$ is prime, $\mu(n)=-1$. The equation with $n$ prime then becomes

$$
a_{1}(s+t+1-\alpha)-a_{2}(s+t+1-\alpha)+(s+1)(t+1)=(t+1) f+g .
$$

We also know that the eigenvalue $(s+t+1-\alpha)$ has multiplicity $\frac{s t(s+1)(t+1)}{\alpha(s+t+1-\alpha)}$. So the sum of the multiplicities of the individual eigenvalues related to this one must be this same value. In the case of $n$ prime, this gives us the equation

$$
a_{1}+(n-1) a_{2}=\frac{s t(s+1)(t+1)}{\alpha(s+t+1-\alpha)}
$$

We solve this system of equations:

$$
\left\{\begin{array}{l}
a_{1} \alpha(s+t+1-\alpha)-a_{2} \alpha(s+t+1-\alpha)=\alpha(t+1) f+\alpha g-\alpha(s+1)(t+1) \\
a_{1} \alpha(s+t+1-\alpha)+a_{2} \alpha(n-1)(s+t+1-\alpha)=s t(s+1)(t+1)
\end{array}\right.
$$

Subtracting the first equation from the second gives:

$$
\begin{array}{r}
a_{2} \alpha n(s+t+1-\alpha)=s t(s+1)(t+1)+\alpha(s+1)(t+1)-\alpha(t+1) f-\alpha g, \\
\text { or } a_{2}=\frac{(s+1)(t+1)(s t+\alpha)-\alpha((t+1) f+g)}{\alpha n(s+t+1-\alpha)}
\end{array}
$$


Substituting this back into equation (2.4) we get

$$
a_{1}+(n-1) \frac{(s+1)(t+1)(s t+\alpha)-\alpha((t+1) f+g)}{\alpha n(s+t+1-\alpha)}=\frac{s t(s+1)(t+1)}{\alpha(s+t+1-\alpha)}
$$

After subtracting and simplifying we get the desired result:

$$
a_{1}=\frac{(s+1)(t+1)(s t-\alpha(n-1))+\alpha(n-1)((t+1) f+g)}{\alpha n(s+t+1-\alpha)}
$$

Using these expressions we can create more expressions for specific cases.

\subsection{Sharply transitive abelian group of automorphisms}

One special type of group of automorphisms is a sharply transitive abelian group. A sharply transitive group contains a unique automorphism mapping each point to every other point. That is, for any two points $x_{i}$ and $x_{j}$ there is exactly one $g$ in the group $G$, that maps $x_{i}$ to $x_{j}$. Consequently, there is only one element of the group that maps any point to itself. Because the group must contain the identity, which maps all points to themselves, there is only one element of $G$ that fixes any points. So elements of $G$ either fix all points or fix no points. Under the action of such a group, the values of $f$ and $g$ in the equation $a_{1}(s+t+1-\alpha)-a_{2}(s+t+1-\alpha)+(s+1)(t+1)=(t+1) f+g$ take on only specific 
values. If the automorphism is the identity then $f=v$ and $g=0$. In all other cases $f=0$. Furthermore, for any nonidentity automorphism, all points will be either collinear with their images or none will. To see this, consider a point $x$ and an automorphism $\theta$ such that $x$ is collinear with its image under $\theta, x^{\theta} \sim x$. Because this group of automorphisms is sharply transitive, there is an element of the the group that maps $x$ to any $y$ in the geometry. Let $\phi$ be the element such that $x^{\phi}=y$. Then $\left(x^{\theta}\right)^{\phi} \sim x^{\phi}$. Because the group is abelian we get $x^{\theta \phi}=x^{\phi \theta}=y^{\theta}$. So $y \sim y^{\theta}$. Thus if one point is mapped to a collinear point, all points are mapped to collinear points. Hence, there are two possible values for $g$; either $g=0$

or $g=v=(s+1) \frac{s t+\alpha}{\alpha}$. For an automorphism of prime order we create the following summary of results. We also include the results for the symmetric case when $s=t$ as this case is of interest later in this thesis. Because each of these values must be integer, these expressions provide us with divisibility conditions for partial geometries that admit a sharply transitive abelian group of automorphisms.

Table 2.1

Divisibility conditions for a partial geometry when acted on by a sharply transitive abelian group of automorphisms.

\begin{tabular}{|c||c|c|}
\hline$s \neq t$ & $a_{1}$ & $a_{2}$ \\
\hline$g=0$ & $\frac{(s+1)(t+1)(\alpha+s t-n \alpha)}{\alpha n(s+t+1-\alpha)}$ & $\frac{(s+1)(t+1)(s t+\alpha)}{\alpha n(s+t+1-\alpha)}$ \\
\hline$g=(s+1)\left(\frac{s t+\alpha}{\alpha}\right)$ & $\frac{t(s+1)(s(n+t)-\alpha(n-1))}{\alpha n(s+t+1-\alpha)}$ & $\frac{t(s+1)(s t+\alpha)}{\alpha n(s+t+1-\alpha)}$ \\
\hline \hline$s=t$ & $\frac{(t+1)^{2}\left(t^{2}-\alpha n+\alpha\right)}{\alpha n(2 t+1-\alpha)}$ & $\frac{(t+1)^{2}\left(t^{2}+\alpha\right)}{\alpha n(2 t+1-\alpha)}$ \\
\hline$g=0$ & $\frac{t(t+1)(t(n+t)-\alpha(n-1))}{\alpha n(2 t+1-\alpha)}$ & $\frac{t(t+1)\left(t^{2}+\alpha\right)}{\alpha n(2 t+1-\alpha)}$ \\
\hline$g=(s+1)\left(\frac{s t+\alpha}{\alpha}\right)$ & &
\end{tabular}


Notice that the value of $n$, the order of the automorphism, is in the denominator of each of these multiplicity values. Because we are only considering prime orders of automorphisms, this means that this prime must be a factor of the numerator in each case. This divisibility condition may provide further insight for the cases considered in the next section of this paper. 



\section{Chapter 3}

\section{Maximal arcs and their generalizations}

\subsection{Maximal arcs}

\subsubsection{Construction of the partial geometry $T_{2}^{*}(\mathcal{K})$}

A finite projective plane is a point-line geometry that satisfies three conditions:

- any two points are incident with exactly one line

- any two lines intersect in exactly one point

- there is a set of four points such that no three points are incident to the same line.

From these axioms it can be deduced that there are the same number of points on each line as there are lines through any point. This value is denoted as $q+1$ where $q$ is referred to as the order of the projective plane. The simplest example of a projective plane is the Fano 
plane. Here the incidence matrix shows clearly that for any two points (rows), there is only

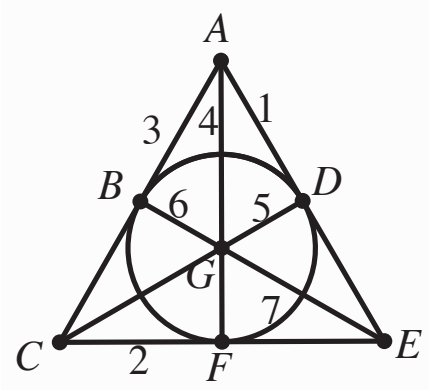

$A$
$B$
$C$
$D$
$E$
$F$
$G$$\quad\left[\begin{array}{lllllll}1 & 2 & 3 & 4 & 5 & 6 & 7 \\ 1 & 0 & 1 & 1 & 0 & 0 & 0 \\ 0 & 0 & 1 & 0 & 0 & 1 & 1 \\ 0 & 1 & 1 & 0 & 1 & 0 & 0 \\ 1 & 0 & 0 & 0 & 1 & 0 & 1 \\ 0 & 1 & 0 & 0 & 0 & 1 & 0 \\ 0 & 0 & 0 & 1 & 0 & 0 & 1 \\ & & 1 & 1 & 1 & 0\end{array}\right]$

Figure 3.1: The Fano plane and its incidence matrix.

one common line (column) and for any two lines (columns) there is only one point (row) incident to both. There is also at least one set of four points (rows) such as $A, B, D, F$ where no line (column) is common to more than two of these points. The incidence matrix also makes it clear the projective plane is of order 2 as each line has three points and each point is on three lines.

A $\{k ; d\}$-arc, $\mathcal{K}$, is a set of $k$ points in a projective plane of order $q$, where $d$ is the maximum number of points in the set that are collinear. In the following discussion we exclude $d=0,1, q, q+1$ as these are trivial cases. If $d=0$ there are no points in the arc and if $d=1$ there is only one point in $\mathcal{K}$. If $d=q$ then there is only one point on each line outside of the arc. Any two points outside of the arc determine a line. Then because this leaves $d-1$ more points on the line, they must also lie outside the arc. So this line contains all of the points that are not in the arc. Thus there is one line outside the arc and all other points inside. Finally if $d=q+1$, the arc contains the entire projective plane. For the more interesting values of $d$, we determine a bound on $k$. 


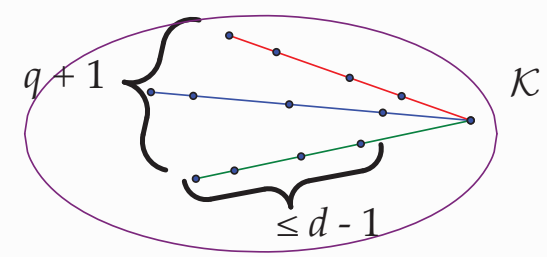

Figure 3.2: Determining an upper bound for $k$.

First consider any point in the arc. There are $q+1$ lines incident with this point. On each of these lines there are at most an extra $d-1$ distinct points in the arc. So the total number of points in the arc is at most $(q+1)(d-1)+1$ where we have added back the point in common to the set of lines. So $k \leq(q+1)(d-1)+1[17]$. When this is an equality, the arc is called maximal. Thus a maximal arc is a nonempty set of $k$ points in the projective plane such that any line intersects the set in either 0 or $d$ points. Expanding the equality we get $k=q d-q+d$.

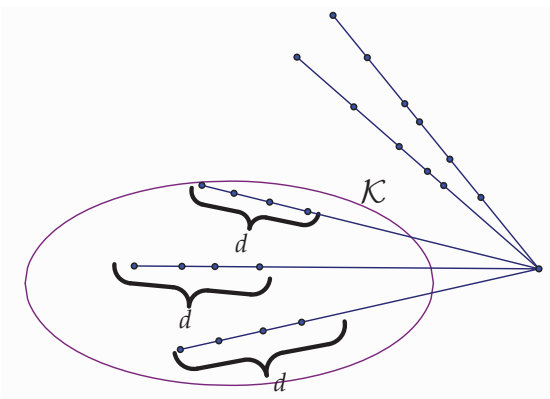

Figure 3.3: The points in $\mathcal{K}$ are in groups of size $d$ in a maximal arc.

As the $k$ points in the arc can be divided into groups of size $d$, we know that $d$ must be a factor of $k$ and consequently a factor of $q d-q+d$. Therefore $d$ must divide $q$. (see Figure 3.3.)

Let $q$ be a prime power and define $P G(n, q)$ to be the geometry where the points are 
one-dimensional vector spaces in $V(n+1, q)$, the lines are two-dimensional vector spaces in $V(n+1, q)$, and in general, $k$-spaces are the $(k+1)$-dimensional vector spaces in $V(n+1, q)$. Here $V(n+1, q)$ is the vector space with rank $n+1$ over $q$ elements. For instance, $P G(2, q)$ is a projective plane of order $q$. Consider a maximal arc $\mathcal{K}$ in $P G(2, q)$ and consider $P G(2, q)$ as a subgeometry of $P G(3, q)$. Define a geometry $T_{2}^{*}(\mathcal{K})$ where

- the points of the geometry are the points of $P G(3, q) \backslash P G(2, q)$,

- the lines of the geometry are the lines of $P G(3, q)$ meeting $P G(2, q)$ in a single point of $\mathcal{K}$.

Lemma $6 T_{2}^{*}(\mathcal{K})$ is a partial geometry $\operatorname{pg}(q-1,(q+1)(d-1), d-1)($ see e.g.[16][17]).

Proof. There are $q$ points on each line so $s=q-1$. In the three-dimensional projective
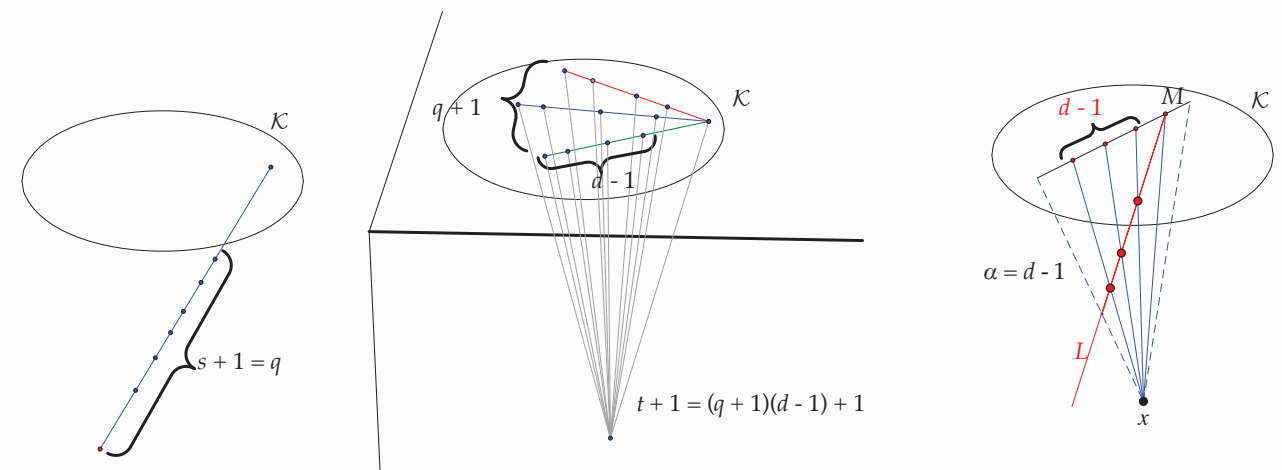

Figure 3.4: Determining the parameters for $T_{2}^{*}(\mathcal{K})$

space, every two points are collinear. So any point in the space below $\mathcal{K}$ reaches each point in $\mathcal{K}$. Thus the number of lines through any point is the same as the number of points in the arc. Because the arc is maximal, this means $t=k-1=(q+1)(d-1)$. Consider a line 
$L$ of this geometry and a point $x$ of the geometry, not on $L$. Then $L$ and $x$ span a plane that intersects $P G(2, q)$ in a line $M$. The intersection $M \cap L$ is a point in $\mathcal{K}$. Hence, $M$ intersects $\mathcal{K}$ in $d-1$ more points. Each one of these points is also on a line through $x$ in the plane spanned by $x$ and $L$. The line $L$ intersects each of these $d-1$ lines. Therefore, $\alpha=d-1$ and $T_{2}^{*}(\mathcal{K})$ is a partial geometry.

\subsubsection{Applying Benson's Theorem}

In the proof of Theorem 5 the expressions obtained for the multiplicities of the eigenvalues were valid for any partial geometry. Using the values $s=q-1, t=(q+1)(d-1)$, and $\alpha=d-1$ we can get expressions for the multiplicities for the eigenvalues associated with this partial geometry. A next natural question is to see if these expressions can provide a simpler proof of previously known conclusions. For instance, it has been proven, that there are no maximal arcs in $P G(2, q)$ when $q$ is odd [1], while numerous examples of maximal $\operatorname{arcs}$ when $q$ is even are known [7].

Consider automorphisms in the form of dilations from one fixed point so $f=1$. Then the remaining points on the lines emanating from this fixed point are mapped to collinear points. Because there are $t+1=(q+1)(d-1)+1$ lines and $q-1$ points on each line that are mapped to collinear points, then $g=(q-1)(q+1)(d-1)+(q-1)$ or $g=\left(q^{2}-1\right)(d-1)+(q-1)$. Making the substitutions for $s, t, \alpha$ in (2.6) and (2.5) we

get $a_{1}=a_{2}=\frac{\left(q^{2}-1\right)(d-q+d q)}{d n}$. Because the dilations form a cyclic group of order 
$q-1$, any automorphism in the group must have an order that is a factor of $q-1$. As we are considering only automorphisms of prime order, this means $n$ is a prime divisor of $q-1$. So $a_{1}=a_{2}$ are guaranteed to be integers and unfortunately the divisibility conditions provide no additional insight here.

\subsection{Generalized maximal arcs}

\subsubsection{Construction of the partial geometry $\mathcal{S}(\mathcal{M})$}

Consider an $m$-dimensional equivalent of an arc in an $n$-dimensional space. To make this concept clearer, we first redefine the original maximal arc. In $P G(2, q)$ the set of points can be thought of as a set of $P G(0, q)$ in a $P G(2, q)$. Each line is a $P G(0+1, q)$. Then a maximal arc is a set of $P G(0, q)$ such that every $P G(0+1, q)$ that contains one of those $P G(0, q)$ intersects or contains exactly $\alpha$ other ones. We now have a definition that extends easily to higher dimensions. A generalized maximal arc, $\mathcal{M}$ in $P G(n, q)$ is a set of disjoint $P G(m, q) \subseteq P G(n, q)$ such that every $P G(m+1, q)$ containing a $P G(m, q) \in \mathcal{M}$ intersects exactly $\alpha$ others. We hope to find a relationship between the parameters $m$ and $n$. DeClerck, Delanote, Hamilton, and Mathon [6] examined a specific case of these structures and found that $2 m+1 \leq n \leq 3 m+2$. We hope to establish a similar relation for the more general case. Consider a generalized maximal arc, $\mathcal{M}$ consisting of $P G(m, q)$ in $P G(n, q)$. Embed $P G(n, q)$ in a $P G(n+1, q)$ and define a geometry $\mathcal{S}(\mathcal{M})$ as follows: 
- the points of the geometry are the points of $P G(n+1, q) \backslash P G(n, q)$.

- the lines of the geometry are the $[m+1]$ spaces that intersect the $P G(n, q)$ in an element of $\mathcal{M}$.

Lemma $7 \mathcal{S}(\mathcal{M})$ is a partial geometry $\operatorname{pg}\left(q^{m+1}-1,|\mathcal{M}|, \alpha\right)$.

Proof. There are $q^{m+1}$ points in each line of $\mathcal{S}(\mathcal{M})$ i.e $[m+1]$ space of $P G(n+1) \backslash$ $P G(n, q)$, so $s+1=q^{m+1}$ is constant. The number of lines through a point is the number of elements ( $[m]$ spaces) in $\mathcal{M}$, so this is also a constant value, $t+1=|\mathcal{M}|$. Let $\mathcal{L}$ be a line of $\mathcal{S}(\mathcal{M})$. (This is an $[m+1]$ space in the projective space.) Let $x$ be a point of the geometry not on this line. Together, $x$ and $\mathcal{L}$ span a $[m+2]$ space in $P G(n+1, q)$ that intersects $P G(n, q)$ in a $[m+1]$ space, $M$.

The intersection of $M$ and $\mathcal{L}$ is an element of $\mathcal{M}$. Hence, $M$ intersects exactly $\alpha$ other

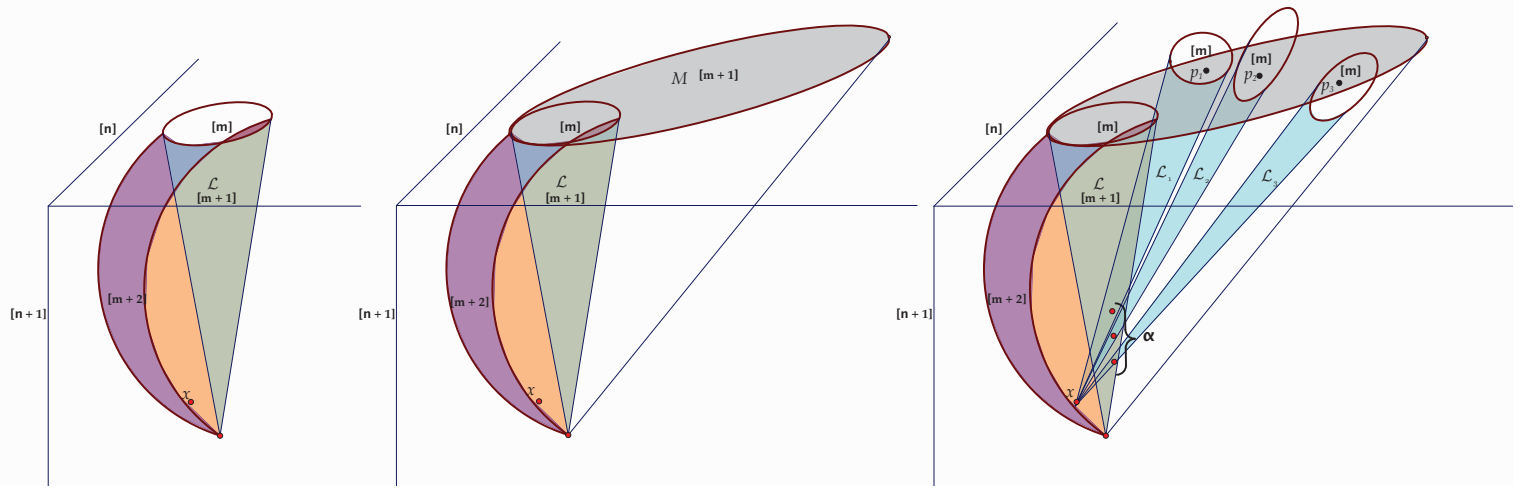

Figure 3.5: The relationship between the spaces.

elements of $\mathcal{M}$. Let $p_{1}, p_{2}, \ldots, p_{\alpha}$ be points, one in each of these other elements of $\mathcal{M}$. Then $x$ and each $p_{i}$ define a line, $\mathcal{L}_{1}, \mathcal{L}_{2}, \ldots, \mathcal{L}_{\alpha}$. These $\mathcal{L}_{i}$ now intersect $\mathcal{L}$ in exactly 
$\alpha$ points. Hence in the geometry $\mathcal{S}$ the point $x$ is collinear with exactly $\alpha$ points on $\mathcal{L}$. Therefore, $\mathcal{S}(\mathcal{M})$ is indeed a partial geometry.

\subsubsection{Applying Benson's Theorem}

Next we establish a necessary condition on $\alpha$ and $t$.

Lemma 8 In the partial geometry $\mathcal{S}(\mathcal{M}), \alpha\left(q^{n-m}-1\right)=t\left(q^{m+1}-1\right)$.

Proof. Using the construction as above, we fix $M$, an element of $\mathcal{M}$. We will count, in two ways, the number of points $p$ such that $p$ is in an element of $\mathcal{M}$, distinct from $M$. First note that $\mathcal{M}$ contains $t$ elements distinct from $M$. Each of these elements ([m] spaces) contains $\frac{q^{m+1}-1}{q-1}$ points. Thus there are $t \frac{q^{m+1}-1}{q-1}$ choices for $p$. For a second count, observe that every $[m+1]$ space containing $M$ contains exactly $\alpha$ of the points we are counting. As there are $\frac{q^{n-m}-1}{q-1}$ such $[m+1]$ spaces, and these spaces intersect exactly in $M$, we obtain $\alpha \frac{q^{n-m}-1}{q-1}$ choices for $p$. Therefore, $\alpha\left(q^{n-m}-1\right)=t\left(q^{m+1}-1\right)$.

We next derive necessary conditions for the existence of a generalized maximal arc. Note that these conditions are the same as the ones obtained in [6]. However, the result here is much stronger than that of [6] as we do not require the generalized maximal arc to satisfy the rather restrictive extra geometric condition as in [6]).

Theorem 9 If $\mathcal{S}(\mathcal{M})$ is constructed from $P G(m, q)$ 's in $P G(n, q)$ where $q$ is a power of 
some prime, $p$, then $n=2 m+1+\frac{m+1}{r+1}$ for some $r$, a non-negative integer, and $\alpha=$ $\frac{\left(p^{k}-1\right)\left(q^{m+1}-1\right)}{q^{n-2 m-1}-1}$ where $0<p^{k}<q^{n-2 m-1}$.

We will prove this through a series of lemmas. First though we recall some of the key relationships.

$$
s+1=q^{m+1}, \quad \frac{s t+\alpha}{\alpha}=q^{n-m}, \quad(s+1)\left(\frac{s t+\alpha}{\alpha}\right)=q^{n+1}
$$

Clearly $s+1 \mid \frac{s t+\alpha}{\alpha}$ so $s+1 \mid s t+\alpha$.

Then adding and subtracting $t$ on the right we get

$$
s+1 \mid s t+\alpha+t-t=t(s+1)+\alpha-t .
$$

Thus $s+1 \mid \alpha-t$ and similarly $s+1 \mid t-\alpha$ which is positive as $\alpha<t$ for a proper partial geometry. We can now write $t=z(s+1)+\alpha$ for some $z \in \mathbb{Z}$. This expression will prove useful in the next lemma.

Lemma 10 In the partial geometry $\mathcal{S}(\mathcal{M}), z q^{m+1}+\alpha+1 \equiv 0\left(\bmod p^{k}\right)$.

Proof. According to Benson's Theorem there exists an automorphism without fixed points that has $g=0$ and one that fixes all points so that $g=v=q^{n+1}$. Hence the relationship 
$(1+t) f+g \equiv(s+1)(t+1)(\bmod s+t+1-\alpha)$ becomes

$$
(s+1)(t+1) \equiv\left\{\begin{array}{cc}
q^{n+1} & (\bmod s+t+1-\alpha) . \\
0 &
\end{array}\right.
$$

Replacing $t+1$ with $z(s+1)+\alpha+1$ we get

$$
(s+1)(z(s+1)+\alpha+1) \equiv\left\{\begin{array}{ccc}
q^{n+1} & \\
0 & (\bmod s+z(s+1)+1)
\end{array}\right.
$$

which reduces to

$$
z(s+1)+\alpha+1 \equiv\left\{\begin{array}{cc}
q^{n+1-m-1} & (\bmod z+1) \\
0 &
\end{array}\right.
$$

Now because $z(s+1)+\alpha+1 \equiv q^{n-m}$ and $z(s+1)+\alpha+1 \equiv 0(\bmod z+1)$, we can say $q^{n-m} \equiv 0(\bmod z+1)$. Thus $z+1 \mid q^{n-m}$. So $z+1$ is a power of $p$. We let $z+1=p^{k}, k>0$. Thus we can now rewrite the congruence $z(s+1)+\alpha+1 \equiv 0(\bmod z+1)$ as $z q^{m+1}+\alpha+$ $1 \equiv 0\left(\bmod p^{k}\right)$

Lemma 11 In the partial geometry $\mathcal{S}(\mathcal{M}), \alpha+1 \equiv 0\left(\bmod p^{k}\right)$

Proof. We begin by rewriting the congruence $z q^{m+1}+\alpha+1 \equiv 0\left(\bmod p^{k}\right)$ as follows:

$$
0 \equiv-z q^{m+1}-q^{m+1}+q^{m+1}-\alpha-1 \equiv 0\left(\bmod p^{k}\right)
$$


Factoring to expose the quantity $z+1$

$$
0 \equiv q^{m+1}-q^{m+1}(z+1)-\alpha-1\left(\bmod p^{k}\right)
$$

Now because $z+1=p^{k}$ this becomes

$$
\begin{gathered}
q^{m+1}-\alpha-1 \equiv 0\left(\bmod p^{k}\right) \\
\text { or } q^{m+1}-(\alpha+1) \equiv 0\left(\bmod p^{k}\right) .
\end{gathered}
$$

Consider the relationship between $p^{k}$ and $q^{m+1}$. If $q^{m+1}<p^{k}$, then $0<q^{m+1}-(\alpha+1)<$ $p^{k}$. Since $q^{m+1}-(\alpha+1) \equiv 0\left(\bmod p^{k}\right)$, then $\alpha+1$ must be equal to $q^{m+1}$. But this is a contradiction as $\alpha+1<s+1=q^{m+1}$.

Therefore $q^{m+1} \geq p^{k}$ and the congruence $q^{m+1}-(\alpha+1) \equiv 0\left(\bmod p^{k}\right)$ implies that $\alpha+1 \equiv 0\left(\bmod p^{k}\right)$

We can now write $\alpha+1=y p^{l}$ where $p \nmid y, l \geq k$, and $p^{l} \leq q^{m+1}$.

Lemma 12 In the partial geometry $\mathcal{S}(\mathcal{M})$,

$$
p^{k} q^{m+1}-p^{k}-q^{m+1}=y p^{l} q^{n-2 m-1}-q^{n-2 m-1}-y p^{l}
$$

Proof. Recall the following relationships:

$$
z=p^{k}-1, \quad s=q^{m+1}-1, \quad \alpha=y p^{l}-1, \quad t=\frac{\alpha\left(q^{n-m}-1\right)}{s} .
$$

We now rewrite the equation $z(s+1)+\alpha=t$, in terms of $p$ and $q$ as

$$
\left(p^{k}-1\right) q^{m+1}+y p^{l}-1=\left(y p^{l}-1\right) \frac{q^{n-m}-1}{q^{m+1}-1} .
$$


Multiplying both sides by $q^{m+1}-1$ :

$$
\left(p^{k}-1\right)\left(q^{2 m+2}-q^{m+1}\right)+y p^{l} q^{m+1}-y p^{l}-q^{m+1}+1=y p^{l} q^{n-m}-q^{n-m}-y p^{l}+1
$$

Expanding and collecting like terms:

$$
p^{k} q^{2 m+2}-p^{k} q^{m+1}-q^{2 m+2}=y p^{l} q^{n-m}-q^{n-m}-y p^{l} q^{m+1}
$$

Dividing both sides by $q^{m+1}$ :

$$
p^{k} q^{m+1}-p^{k}-q^{m+1}=y p^{l} q^{n-2 m-1}-q^{n-2 m-1}-y p^{l} .
$$

We next consider the relationship between $l$ and $k$.

Lemma 13 Under the conditions of Lemma 12, $l=k$.

Proof. Recall now that $p$ divides $q$. Also recall $p^{l}<q^{m+1}$.

In Lemma 11 we established that $l \geq k$. Now suppose $l>k$.

Consider (3.1) $\bmod p^{l}$. Since $p^{l}<q^{m+1}$ this equation becomes the congruence $p^{k} \equiv$ $q^{n-2 m-1}\left(\bmod p^{l}\right)$. But because $k<l$, we know $p^{k} \not \equiv 0\left(\bmod p^{l}\right)$. Thus $q^{n-2 m-1}<p^{l}$. So since both $p^{k}$ and $q^{n-2 m-1}$ are less than $p^{l}$, then they must be equal. That is, $p^{k}=q^{n-2 m-1}$. 
Substituting this in (3.1) we get

$$
q^{n-m}-q^{n-2 m-1}-q^{m+1}=y p^{l} q^{n-2 m-1}-q^{n-2 m-1}-y p^{l}
$$

Combining like terms and then factoring gives us:

$$
q^{m+1}\left(q^{n-2 m-1}-1\right)=y p^{l}\left(q^{n-2 m-1}-1\right)
$$

This implies that $q^{m+1}=y p^{l}$. But this is the same as $s+1=\alpha+1$ which is not possible as $\alpha<s$. Therefore, $l=k$. Note that $q^{n-2 m-1}-1 \neq 0$. If it were, then the congruence $p^{k} \equiv q^{n-2 m-1}\left(\bmod p^{l}\right)$ would imply $p^{k} \equiv 1\left(\bmod p^{l}\right)$ which is not possible.

Now (3.1) becomes

$$
p^{k} q^{m+1}-p^{k}-q^{m+1}=y p^{k} q^{n-2 m-1}-q^{n-2 m-1}-y p^{k} .
$$

Before considering the relationship between $p^{k}$ and $q^{n-2 m-1}$ we prove a general lemma.

Lemma 14 The expression $\frac{\left(q^{a}-1\right)\left(q^{b}-1\right)}{q^{c}-1}$ is integer valued if and only if either $q^{c}-1 \mid$ $q^{a}-1$ or $q^{c}-1 \mid q^{b}-1$.

Proof. $(\Leftarrow)$ is obvious, $(\Rightarrow)$ Let $\alpha=\operatorname{gcd}(a, c)$ and $\beta=\operatorname{gcd}(b, c)$.

Then $\operatorname{gcd}\left(q^{a}-1, q^{c}-1\right)=q^{\alpha}-1$ and $\operatorname{gcd}\left(q^{b}-1, q^{c}-1\right)=q^{\beta}-1$.

Now if $g^{c}-1 \mid\left(g^{a}-1\right)\left(g^{b}-1\right)$, then $g^{c}-1 \mid\left(g^{\alpha}-1\right)\left(g^{\beta}-1\right)=g^{\alpha+\beta}-g^{\alpha}-g^{\beta}+1$. 
This implies that $\alpha+\beta>c$. Without loss of generality, we can then assume $\alpha>\frac{c}{2}$. But we know $g^{\alpha}-1 \mid g^{c}-1$ because $\alpha \mid c$. The only way to satisfy both of these conditions is for $\alpha=c$. This implies $q^{c}-1 \mid q^{a}-1$. By symmetry, if $\beta=c$, then $q^{c}-1 \mid q^{b}-1$. Thus $\frac{\left(q^{a}-1\right)\left(q^{b}-1\right)}{q^{c}-1}$ is integer valued if and only if either $q^{c}-1 \mid q^{a}-1$ or $q^{c}-1 \mid q^{b}-1$.

We now turn to the relationship between $n$ and $m$.

Lemma 15 Under the conditions of Lemma 13, $2 m+1<n \leq 2.5 m+1.5$ or $n=3 m+2$.

Proof. We investigate this by considering the relationship between $p^{k}$ and $q^{n-2 m-1}$ as separate cases.

Suppose $p^{k}=q^{n-2 m-1}$. Making this substitution in (3.2) we get

$$
q^{n-m}-q^{m+1}-q^{n-2 m-1}=y p^{k} q^{n-2 m-1}-q^{n-2 m-1}-y p^{k} .
$$

Combining like terms and factoring leads us to

$$
q^{m+1}\left(q^{n-2 m-1}-1\right)=y p^{k}\left(q^{n-2 m-1}-1\right) .
$$

So $q^{m+1}=y q^{n-2 m-1}$ which implies $y=1$ and thus $n=3 m+2$.

In this case $\alpha=q^{m+1}-1=s$ which though not a proper partial geometry, is still acceptable as an upper limit on $n$.

Suppose $p^{k}<q^{n-2 m-1}$

We now use a result from De Winter [8] that allows us to assume without loss of generality that $q$ is prime, or equivalently, that $p=q$. We know that a $P G(n, q)$ is in one to one 
correspondence with the $(n+1)$-dimensional vector space $V(n+1, q)$ over the finite field $\mathbb{F}_{q}$ as each vector line in $V(n+1, q)$ determines a point in $P G(n, q)$. If $q=p^{d}$ then $\mathbb{F}_{q}$ is itself a $d$-dimensional vector space over $\mathbb{F}_{p}$. Hence $V(n+1, q)$ can be thought of as a $d(n+1)$-dimensional vector space $V(d(n+1), p)$ over $\mathbb{F}_{p}$. This is then in one to one correspondence to the $(d(n+1)-1)$-dimensional projective space $P G(d(n+1)-1, p)$. This field reduction as described in [8] provides a natural map from substructures of $P G(n, q)$ to substructures of $P G(d(n+1)-1, p)$. In our case, every $P G(m, q) \subset P G(n, q)$ will naturally be mapped to some $P G(d(m+1)-1, p) \subset P G(d(n+1)-1, p)$. The special case of a more general result from [8] states that:

If $\mathcal{K}$ is a generalized maximal arc consisting of $P G(m, q)$ in $P G(n, q)$, then the image of $\mathcal{K}$ under field reduction is a generalized maximal arc consisting of $P G(d(m+1)-1, p)$ in $P G(d(n+1)-1, p)$. Moreover, this image has the same $\alpha$ value as $\mathcal{K}$.

It is worth noting that replacing $n$ with $d(n+1)-1$ and $m$ with $d(m+1)-1$, the original statement in the theorem remains the same after some simplification. Now we can assume without loss of generality that $p=q$. Doing this (3.1) becomes

$$
q^{m+1+k}-q^{m+1}-q^{k}=y q^{k+n-2 m-1}-q^{n-2 m-1}-y q^{k}
$$

Dividing by $q^{k}$ we get

$$
q^{m+1}-q^{m+1-k}-1=y q^{n-2 m-1}-q^{n-2 m-1-k}-y .
$$


Solving this equation for $y$ we get

$$
y=\frac{q^{m+1}-q^{m+1-k}+q^{n-2 m-1-k}-q^{n-2 m-1}+q^{n-2 m-1}-1}{q^{n-2 m-1}-1} .
$$

Note that by adding and subtracting $q^{n-2 m-1}$ in the numerator we now have an expression that can be simplified to

$$
y=1+\frac{\left(q^{k}-1\right)\left(q^{m+1-k}-q^{n-2 m-1-k}\right)}{q^{n-2 m-1}-1} .
$$

Using the assumption that $k<n-2 m-1$ we can factor further to get

$$
y=1+\frac{q^{n-2 m-1-k}\left(q^{k}-1\right)\left(q^{3 m-n+2}-1\right)}{q^{n-2 m-1}-1} .
$$

Using lemma(14) and the fact that $k \neq 0$ we conclude that either

$$
q^{n-2 m-1}-1 \mid q^{k}-1 \text { or } q^{n-2 m-1}-1 \mid q^{3 m-n+2}-1
$$

By assumption, $k<n-2 m-1$ so that means $q^{n-2 m-1}-1 \mid q^{3 m-n+2}-1$.

Thus $n-2 m-1 \mid 3 m-n+2$ and either $n-2 m-1 \leq 3 m-n+2$ or $3 m-n+2=0$. Solving for $n$ we get $n \leq 2.5 m+1.5$ or $n=3 m+2$.

So while not giving a precise relationship between $n$ and $m$, we do have a bound on the value of $n$ for any given $m$. We also have a divisibility condition that we can use to determine sets of values $n, m, k$ that satisfy all of our assumptions. For example, if we consider $n=2.5 m+$ 
1.5 , the values $n=9, m=3, k=1$ satisfy both $n-2 m-1 \mid 3 m-n+2$ and $k<n-2 m-1$.

Other sets are listed in Table 3.1:

\section{Table 3.1}

sets of values for $n, m$ and $k$

\begin{tabular}{|c|c|c|c|c|}
$n$ & $m$ & $n-2 m-1$ & $k$ & $3 m-n+2$ \\
\hline 9 & 3 & 2 & 1 & 2 \\
\hline 14 & 5 & 3 & 1 & 3 \\
\hline 14 & 5 & 3 & 2 & 3 \\
\hline 19 & 7 & 4 & 1 & 4 \\
\hline 19 & 7 & 4 & 2 & 4 \\
\hline 19 & 7 & 4 & 3 & 4 \\
\hline 13 & 5 & 2 & 1 & 4 \\
\hline 20 & 8 & 3 & 1 & 6 \\
\hline 20 & 8 & 3 & 2 & 6 \\
\hline
\end{tabular}

Now using the value for $y$ obtained above, we can rewrite the values of $s, t, \alpha$.

$$
s=q^{m+1}-1, \quad t=\alpha \frac{q^{n-m}-1}{q^{m+1}-1}, \quad \alpha+1=y q^{k} \Rightarrow \alpha=\frac{\left(q^{k}-1\right)\left(q^{m+1}-1\right)}{q^{n-2 m-1}-1} .
$$

In the case of $n=3 m+2$, these expressions become

$$
s=q^{m+1}-1, \quad t=\alpha\left(q^{m+1}-1\right), \quad \alpha=\left(q^{k}-1\right) .
$$

Each of the sets of values above produce integer values for $s, t, \alpha$ as well as satisfying the divisibility conditions discussed earlier. Thus while not disproving the existence of geometries with $k<n-2 m-1$, we have limited the possibilities for this range of values 
by the divisibility condition, $n-2 m-1 \mid 3 m-n-2$. We can also express this condition as the equation $r(n-2 m-1)=3 m-n-2$ for some $r \in \mathbb{Z}$. Solving for $n$ we get $n=2 m+1+$ $\frac{m+1}{r+1}$. While offering no new information, it presents us with another way to view the same information allowing us to place the value for $n$ in the interval $2 m+1<n \leq 2.5 m+1.5$ or $n=3 m+2$.

The final case to consider is if $p^{k}>q^{n-2 m-1}$. Again we assume without loss of generality, that $p=q$. Also, note that now $3 m+2 \geq n$ or equivalently, $m+1 \geq n-2 m-1$.

Beginning with $q^{m+1+k}-q^{m+1}-q^{k}=y q^{k+n-2 m-1}-q^{n-2 m-1}-y q^{k}$ as before, this time we divide by $q^{n-2 m-1}$ to get

$$
q^{-n+3 m+2+k}-q^{-n+3 m+2}-q^{k-n+2 m+1}=y q^{k}-1-y q^{k-n+2 m+1} .
$$

Solving for $y$ gives us

$$
y=\frac{q^{-n+3 m+2+k}-q^{-n+3 m+2}-q^{k-n+2 m+1}+1}{q^{k}-q^{k-n+2 m+1}} .
$$

Adding and subtracting $q^{k}$ in the numerator this becomes

$$
y=1+\frac{q^{-n+3 m+2+k}-q^{-n+3 m+2}+1-q^{k}}{q^{k}-q^{k-n+2 m+1}} .
$$

which we can factor to be

$$
y=1+\frac{\left(q^{k}-1\right)\left(q^{-n+3 m+2}-1\right)}{q^{k-n+2 m+1}\left(q^{n-2 m-1}-1\right)} .
$$

The fact that $3 m+2 \geq n$ ensures that the exponent in the numerator is positive. Now the numerator of this fraction is not a power of $q$ so we have reached a contradiction as $y$ will not be an integer under our assumption. Thus the assumption that $k>n-2 m-1$ cannot be true. 
Therefore, either $n=3 m+2$ or $2 m+1<n \leq 2.5 m+1.5$ where $n-2 m-1 \mid 3 m-n-2$.

\section{Proof of Theorem 9}

By Lemma 10 and 11 we know $\alpha+1=y p^{k}$. The equation from Lemma 12 with the substitution from Lemma 13 leads us to the equation $p^{k} q^{m+1}-q^{m+1}-p^{k}=$ $y p^{k} q^{n-2 m-1}-q^{n-2 m-1}-y p^{k}$. Lemma 15 leads us to the conclusion that either $n=3 m+2$ and $\alpha=q^{k}-1$ which is not a proper partial geometry, or $\alpha=\frac{\left(p^{k}-1\right)\left(q^{m+1}-1\right)}{q^{n-2 m-1}-1}$ and the consequence that $n=2 m+1+\frac{m+1}{r+1}$ for some non-negative integer $r$, proving the theorem. 



\section{Chapter 4}

\section{Partial geometries with an abelian}

\section{Singer group}

In this section we begin by turning our focus from the properties of a partial geometry and the adjacency matrix of its point graph to looking at partial geometries and groups of automorphisms acting on those geometries. Specifically we focus on the actions of sharply transitive abelian groups. Sharply transitive groups automorphisms acting on geometries are generally referred to as Singer groups in honor of James Singer who studied the actions of such groups on projective planes [14].

Assume $S$ is a proper partial geometry and $G$ is an abelian group of automorphisms acting sharply transitively on it. First note that if an element of $G$ fixes one point, then it fixes all points and thus is the identity. Then considering non-identity automorphisms, the value of $f$ in Theorem 4 is zero. Using this information Theorem 4 gives us 
the congruence $(s+1) \frac{(s t+\alpha)}{\alpha} \equiv(s+1)(t+1)(\bmod s+t+1-\alpha) . \quad$ If we then consider $g \in G$ such that no point is mapped to a collinear point, Theorem 4 gives us $(s+1)(t+1) \equiv 0(\bmod s+t+1-\alpha)$

\subsection{Classifying partial geometries based on stabilizer size}

Next consider how many elements of $G$ stabilize any line in $S$. We know that the identity stabilizes everything in the geometry. So $\left|\operatorname{Stab}_{G}(L)\right|$ is at least 1 . To determine what other values there are for $\left|\operatorname{Stab}_{G}(L)\right|$, assume $g \in \operatorname{Stab}_{G}(L)$, and let $x$ be a point on $L$. Then $x^{g}$, the image of $x$ under $g$, is also on $L$ and $L^{g}=L$. Let $y=x^{h}$ for some $h \in G \backslash\{i d\}$ with $x^{h}$ on $L$ and distinct from both $x$ and $x^{g}$. We can then express $L$ as $\left\langle x, x^{g}\right\rangle$. Applying $h$ to this second expression of $L$ gives us $\left\langle x^{h}, x^{g h}\right\rangle$. However because $G$ is an abelian group, we can also write this as $\left\langle x^{h}, x^{h g}\right\rangle$. But this is the same as $\left\langle y, y^{g}\right\rangle=L$. Thus $h$ also stabilizes $L$. Because there are $s+1$ points on $L,\left|\operatorname{Stab}_{G}(L)\right|=s+1$. Thus $\left|\operatorname{Stab}_{G}(L)\right|=1$ or $\left|\operatorname{Stab}_{G}(L)\right|=s+1$. These observations led DeWinter to classify the partial geometries with an abelian Singer group into three types: "spread type if $\left|\operatorname{Stab}_{G}(L)\right|=s+1$ for each line $L$ of $S ; \ldots$. rigid type if $\left|\operatorname{Stab}_{G}(L)\right|=1$ for each line $L$ of $S ; \ldots$ mixed type otherwise" [10].

Concerning pairs $(S, G)$, of the spread-type, where $S$ is the geometry and $G$ is a sharply transitive group of automorphisms acting on the geometry, DeWinter [9] showed in a separate paper that when $\alpha=1$, every finite generalized quadrangle that admits an abelian 
Singer group is of spread-type. In [10] he characterized the geometries with $\alpha=2$ that are of spread type. Cases of $\alpha>2$ have not yet been studied at this level of detail.

Another consideration for pairs $(S, G)$ of this type is to generalize the results to higher dimensional spaces. The partial geometries discussed in the previous chapter are all examples of this spread type.

Pairs $(S, G)$ of the mixed type have been considered by DeWinter [10] as well as Leung, Ma, and Schmidt [13]. The latter group made great progress toward proving there are no partial geometries of the mixed-type for $\alpha=2$. However no work has been done with larger values of $\alpha$.

\subsubsection{Possible parameters for rigid type geometries}

Concerning $(S, G)$ pairs of the rigid type, it has been proven that there is only one proper partial geometry, $\operatorname{pg}(5,5,2)$ when $\alpha=2[10]$. We will expand on the methods used in that proof to discover possible parameters for geometries when $\alpha>2$. In the rigid case, we can deduce an additional relationship between the parameters $s$ and $t$. Consider a line $L$ with points $p_{0}, p_{1}, p_{2}, \ldots, p_{s}$ on $L$. Because the group of automorphisms is sharply transitive, there is a $g_{i} \in G$ such that $p_{i}^{g_{i}}=p_{0}$ for each $i=0,1,2, \ldots, s$. Then because there are $s+1$ points on a line, there are $s+1$ lines through $p_{0}$ that are the image of $L$ under the corresponding $g_{i}$. Now if $t>s$ there are more lines through $p_{0}$. Thus the $t+1$ lines through $p_{0}$ can be split into groups of size $s+1$ and $t+1=x(s+1)$ for some integer $x$. 


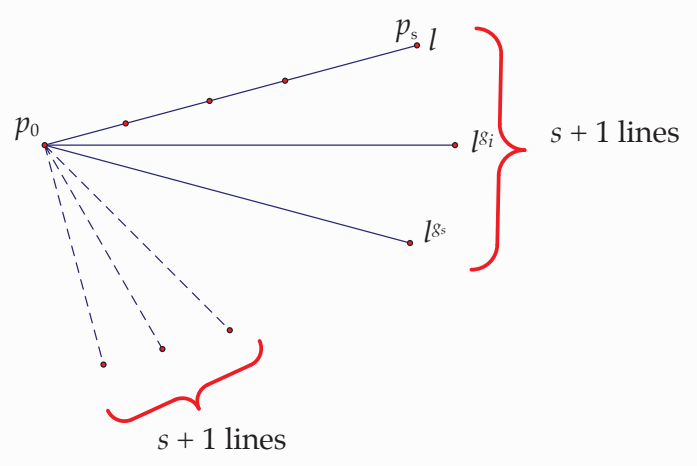

Figure 4.1: The lines through $p_{0}$ are in groups of size $s+1$

Combining this equation with the condition from Theorem $4,(s+1)(t+1) \equiv 0(\bmod s+$ $t+1-\alpha)$ gives us the congruence

$$
x(s+1)^{2} \equiv 0(\bmod (x+1)(s+1)-(1+\alpha))
$$

From this congruence we can determine a bound on the value of $s$ for any given value of $\alpha$. Multiplying by $(x+1)^{2}$

$$
x((x+1)(s+1))^{2} \equiv 0(\bmod (x+1)(s+1)-(1+\alpha))
$$

Subtracting $(\alpha+1)^{2} x$

$$
x((x+1)(s+1))^{2}-(\alpha+1)^{2} x \equiv-(\alpha+1)^{2} x(\bmod (x+1)(s+1)-(1+\alpha))
$$


Factoring as a difference of squares

$$
\begin{aligned}
& x((x+1)(s+1)-(\alpha+1))((x+1)(s+1)+(\alpha+1)) \\
& \equiv-(\alpha+1)^{2} x(\bmod (x+1)(s+1)-(1+\alpha)) .
\end{aligned}
$$

Because $(x+1)(s+1)-(\alpha+1) \equiv 0(\bmod (x+1)(s+1)-(1+\alpha))$ we get

$$
(\alpha+1)^{2} x \equiv 0(\bmod (x+1)(s+1)-(1+\alpha)) .
$$

So $(\alpha+1)^{2} x \geq(x+1)(s+1)-(1+\alpha)=s x+x+s-\alpha$.

Subtracting $x$

$$
\left((\alpha+1)^{2}-1\right) x \geq s x+(s-\alpha)
$$

We know $\alpha<\min (s, t)$ so $s-\alpha>0$. Thus for $\left((\alpha+1)^{2}-1\right) x$ to be greater than $s x+(s-\alpha)$ we must have $s<(\alpha+1)^{2}-1$. So for any chosen $\alpha$, we can find the values of $s$ and $t$ that form a potential partial geometry. The Mathematica code below searches for these sets of parameters and also prints the parameters of their associated strongly regular graphs.

$$
\begin{aligned}
& \operatorname{For}[\alpha=2, \alpha<9, \alpha++, \\
& \operatorname{For}\left[s=\alpha+1, s<(\alpha+1)^{2}, s++, c=\operatorname{Ceiling}\left[(\alpha+1)^{2} /(s+1)-1\right] ;\right. \\
& \operatorname{For}\left[x=1, x \leq(s-\alpha) * c /\left((\alpha+1)^{2}-(s+1) * c\right), x++,\right. \\
& \operatorname{If}\left[\operatorname{Mod}\left[(\alpha+1)^{2} * x,(s+1) * x+s-\alpha\right]==0, t=(s+1) * x-1 ;\right. \\
& \operatorname{If}[\operatorname{Mod}[(s+1) *(t+1), s+t-\alpha+1]==0, v=(s+1) *(s * t+\alpha) / \alpha ;
\end{aligned}
$$




$$
\begin{aligned}
& \operatorname{If}[\operatorname{Mod}[v, s+t-\alpha+1]==0, k=s *(t+1) ; \lambda=s-1+t *(\alpha-1) ; \mu=\alpha *(t+1) ;
\end{aligned}
$$

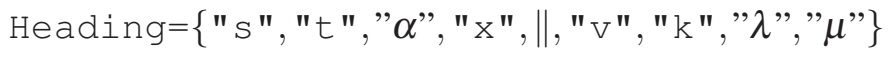

$$
\begin{aligned}
& \text { Print [MatrixForm[ }[\text { Heading, }\{s, t, \alpha, x, " \| ”, v, k, \lambda, \mu\}\}]] \text {; } \\
& \operatorname{If}[\operatorname{PrimeNu}[v]==1, \\
& \text { Print }[\mathrm{v}=\text { Superscript@@@FactorInteger }[v]] \text {, } \\
& \text { Print }[\mathrm{v}=\text { CenterDot@@(Superscript@@@FactorInteger[v] })]]]]]]]]
\end{aligned}
$$

Table 4.1 shows the sets produced for $2 \leq \alpha \leq 8$.

Looking at the sets of values in Table 4.1 prompted the following Lemmas.

Table 4.1

Values of $s, t, \alpha$ for potential partial geometries.

\begin{tabular}{|c|c|c|c|c|c|c|c}
$s$ & $t$ & $\alpha$ & $x$ & $v$ & $k$ & $\lambda$ & $\mu$ \\
\hline 5 & 5 & 2 & 1 & $81=3^{4}$ & 30 & 9 & 12 \\
\hline 11 & 23 & 3 & 2 & $1024=2^{10}$ & 264 & 56 & 72 \\
\hline 14 & 14 & 4 & 1 & $750=2 \cdot 3^{3} \cdot 5^{3}$ & 210 & 55 & 60 \\
\hline 19 & 59 & 4 & 3 & $5625=3^{2} \cdot 5^{4}$ & 1140 & 195 & 240 \\
\hline 29 & 119 & 5 & 4 & $20736=2^{8} \cdot 3^{4}$ & 3480 & 504 & 600 \\
\hline 27 & 27 & 6 & 1 & $3430=2 \cdot 5 \cdot 7^{3}$ & 756 & 161 & 168 \\
\hline 34 & 69 & 6 & 2 & $13720=2^{3} \cdot 5 \cdot 7^{3}$ & 2380 & 378 & 420 \\
\hline 41 & 209 & 6 & 5 & $60025=5^{2} \cdot 7^{4}$ & 8610 & 1085 & 1260 \\
\hline 55 & 335 & 7 & 6 & $147456=2^{14} \cdot 3^{2}$ & 18480 & 2064 & 2352 \\
\hline 44 & 44 & 8 & 1 & $10935=3^{7} \cdot 5$ & 1980 & 351 & 360 \\
\hline 62 & 188 & 8 & 3 & $91854=2 \cdot 3^{8} \cdot 7$ & 11718 & 1377 & 1512 \\
\hline 71 & 503 & 8 & 7 & $321489=3^{8} \cdot 7^{2}$ & 35784 & 3591 & 4032
\end{tabular}

Lemma $16 \mu$ is always even.

Proof. $\mu=\alpha(t+1)$

If $s$ is odd, then $t$ must be odd. This is because $\frac{t+1}{s+1}=x$ must be an integer and if $s+1$ is 
even, then $t+1$ must also be even. So $\mu=\alpha(t+1)$ is even.

Suppose $s$ is even.

Case 1: Assume $t$ is even. Then $(s+1)(t+1)$ is odd. If $(s+1)(t+1) \equiv 0(\bmod s+t+1-$ $\alpha)$, then $s+t+1-\alpha$ must also be odd. This implies $\alpha$ is even. Thus $\mu=\alpha(t+1)$ is even. Case 2: Assume $t$ is odd. Then $\mu=\alpha(t+1)$ is even.

So $\mu$ is always even.

Lemma 17 For every even value of $\alpha$ there is a set of parameters for a potential partial geometry $(s=t, t, \alpha)$.

Proof. Let $\alpha$ be even and $s=t$.

Then $(t+1) \frac{t^{2}+\alpha}{\alpha} \equiv 0(\bmod 2 t+1-\alpha)$ and $(t+1)^{2} \equiv 0(\bmod 2 t+1-\alpha)$, so

$$
(t+1) \frac{t^{2}+\alpha}{\alpha} \equiv(t+1)^{2}(\bmod 2 t+1-\alpha)
$$

Multiplying by $\alpha$ we get

$$
(t+1)\left(t^{2}+\alpha\right) \equiv \alpha(t+1)^{2}(\bmod 2 t+1-\alpha)
$$

Then subtracting we get

$$
(t+1)\left(t^{2}+\alpha-\alpha(t+1)\right) \equiv 0(\bmod 2 t+1-\alpha)
$$


Simplifying this gives us

$$
t(t+1)(t-\alpha) \equiv 0(\bmod 2 t+1-\alpha)
$$

Suppose $t \not \equiv 0(\bmod 2 t+1-\alpha)$. Then we may rewrite the congruence as

$$
t^{2}+(1-\alpha) t-\alpha \equiv 0(\bmod 2 t+1-\alpha)
$$

Multiplying by $(\alpha-1)$ we get

$$
t^{2}(\alpha-1)-t(\alpha-1)^{2}-\alpha(\alpha-1) \equiv 0(\bmod 2 t+1-\alpha) .
$$

When working in $(\bmod 2 t+1-\alpha)$ we have $\alpha-1=2 t$. So we can rewrite the above congruence as:

$$
t^{2}(\alpha-1)-t(\alpha-1)^{2}-2 \alpha t \equiv 0(\bmod 2 t+1-\alpha) .
$$

Then factoring out $t$ we get

$$
t\left(t(\alpha-1)-(\alpha-1)^{2}-2 \alpha\right) \equiv 0(\bmod 2 t+1-\alpha) .
$$


Once again removing the factor of $t$, and then expanding and simplifying this becomes

$$
(\alpha-1) t-\alpha^{2}-1 \equiv 0(\bmod 2 t+1-\alpha)
$$

Now we know $\alpha$ is even, so let $\alpha=2 k$.

Then $\alpha-2=2(k-1)$, and after multiplying by $t$ we get

$$
(\alpha-2) t=2(k-1) t
$$

Now substituting $\alpha-1$ for $2 t$, this becomes

$$
(\alpha-2) t=(k-1)(\alpha-1)
$$

But $k-1=\frac{\alpha-2}{2}$ so we get

$$
(\alpha-2) t=\frac{\alpha^{2}-3 \alpha+2}{2}
$$

Now $(\alpha-1) t=(\alpha-2) t+t$. We can use this along with the expression for $(\alpha-2) t$ to rewrite our equivalence as:

$$
t+\frac{\alpha^{2}-3 \alpha+2}{2}-\alpha^{2}-1 \equiv 0(\bmod 2 t+1-\alpha)
$$


This simplifies to

$$
t-\frac{\alpha^{2}+3 \alpha}{2} \equiv 0(\bmod 2 t+1-\alpha)
$$

Therefore $t=\frac{\alpha^{2}+3 \alpha}{2}$ is a solution for $t$ whenever $\alpha$ is even. Thus for every even value of $\alpha$, there is a set of parameters for a potential partial geometry $(s=t, t, \alpha)$.

The sets of values in Table 4.1 were also investigated using the expressions for the eigenvalues and each produced integer values when each prime factor of $v$ was used as $n$. Each set also satisfied the congruences from Theorem 4.

Of the values in the table, only the first set $(5,5,2)$ is currently known to be a partial geometry. Because there are an infinite set of possible parameters in the $s=t$ case, we choose to investigate the potential $p g(14,14,4)$ in the hopes that insights gained there might be of use in the larger class. So we hypothesize the existence of $p g(14,14,4)$ and try to deduce its structure.

\subsection{Observations on a hypothetical geometry}

Let $S$ denote the point set of the $p g(14,14,4)$ and $G$ an abelian automorphism group acting regularly on the points of $S$. That is, each element of $G$ maps points of $S$ to other points in $S$. Let $x$ be a fixed point in $S$ and identify $x$ with $i d \in G$. Also, identify the point $y \in S$ with the unique $g$ in $G$ such that $x^{g}=y$. This gives us a one-to-one correspondence between $S$ and $G$. So now instead of working with $S$, we will work with $G$. This geometry is associated with 
a $\operatorname{srg}(750,210,55,60)$. Factoring the number of vertices as $2 \cdot 3 \cdot 5^{3}$ gives our first insights into the structure. As any fixed point can be mapped to each of the 750 points, there are also 750 elements of $G$. Let $D$ denote the set of all elements of $G$ that are collinear with the identity, $i d$. That is, the automorphisms that map $x$ to points collinear with $x$. We can thus create an image to help us understand the relationships as below:

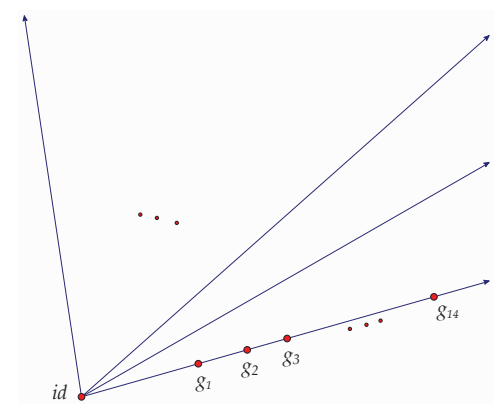

Figure 4.2: The set $D$

Observation 1 There is one element of order 2 and it is not in $D$.

We know there is one element of order 2 as 2 is a factor in the order of $D$. There is only one such element because of the structure of finite abelian groups. The prime factorization $2 \cdot 3 \cdot 5^{3}$ means this group is either $\mathbb{Z}_{2} \times \mathbb{Z}_{3} \times \mathbb{Z}_{5^{3}}$ or $\mathbb{Z}_{2} \times \mathbb{Z}_{3} \times \mathbb{Z}_{5} \times \mathbb{Z}_{5^{2}}$ or $\mathbb{Z}_{2} \times \mathbb{Z}_{3} \times \mathbb{Z}_{5} \times \mathbb{Z}_{5} \times \mathbb{Z}_{5}$. In any of these cases, there is only one factor of order 2 . Suppose $g_{1}$ is the point with order 2 and is in $D$. Applying $g_{1}$ to the points on the first line $i d$ is mapped to $g_{1}$ and $g_{1}$ is mapped to $i d$. Thus all of the points on the line remain fixed. This is not allowed and thus the point of order 2 cannot be in $D$. 
Observation 2 There is one element of order 3.

Again by the same reasoning as in observation 1, we know this because 3 is a factor of the number of vertices. We do not know yet whether or not the element of order 3 is in $D$.

Observation 3 Let $L$ be a line through $i d$ and let its remaining points be $g_{1}, g_{2}, \ldots, g_{14}$. Then the $g_{i}^{-1}$ are two by two collinear.

Let $g_{j}^{-1}$ act on $L$. Because $g_{j}^{-1} g_{j}=i d$ and $g_{j}^{-1} g_{i}=g_{i} g_{j}^{-1}$, we know that $g_{i} g_{j}^{-1} \sim i d$. Now let $g_{i}^{-1}$ act on $g_{i} g_{j}^{-1}$ and $i d$. This tells us that $g_{j}^{-1} \sim g_{i}^{-1}$. Now suppose $g_{i}^{-1} \sim g_{j}^{-1} \sim g_{k}^{-1}$.

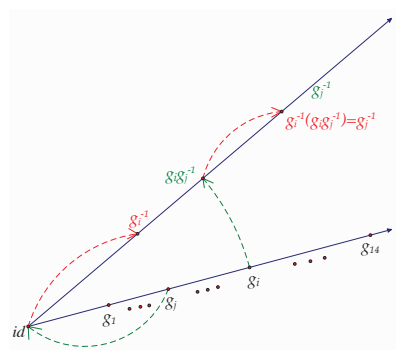

Figure 4.3: The action of $g_{j}^{-1}$ and then $g_{i}^{-1}$

Let $g_{i}$ act on these points. Then $i d \sim g_{i} g_{j}^{-1} \sim g_{i} g_{k}^{-1}$ were all on a line. But $g_{i} g_{j}^{-1}, g_{i} g_{k}^{-1}$ are on distinct lines through $i d$. Therefore the original three points are not on a line and the

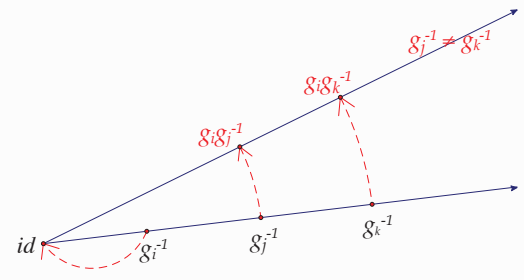

Figure 4.4: The action of $g_{i}$ on inverses. 
$g_{i}^{-1}$ are two by two collinear.

Observation 4 Two points, $g$ and $h$ are collinear if and only if $g^{-1} h \in D$.

$\Rightarrow$

Suppose $g \sim h$ on $L$. Let $g^{-1}$ act on $L$. Then $g \mapsto i d$ and $h \mapsto g^{-1} h$. so $g^{-1} h \sim i d$ and $g^{-1} h \in D$.

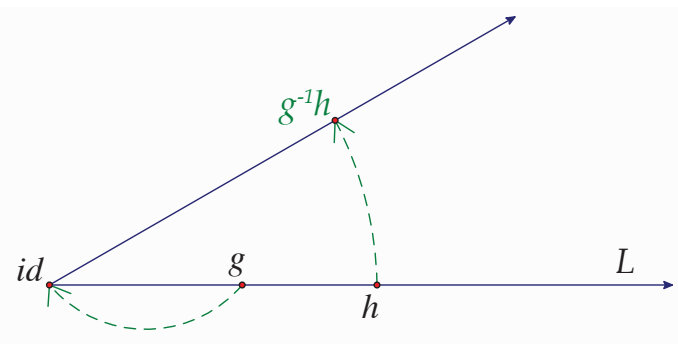

Figure 4.5: The action of $g^{-1}$.

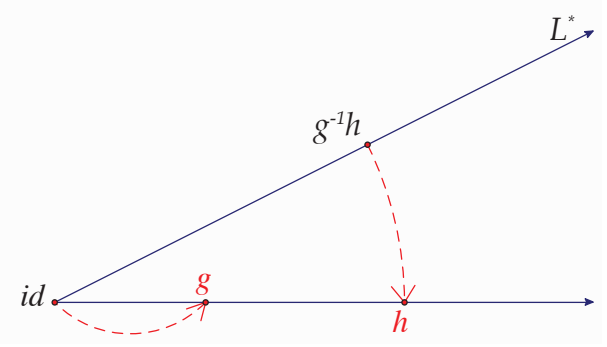

The action of $g$.

$\Leftarrow$

Suppose $g^{-1} h \in D$. Then $g^{-1} h \sim i d$ on some line $L^{*}$. Let $g$ act on $L^{*}$. Then $g^{-1} h \mapsto h$, and $i d \mapsto g$ so $g \sim h$.

Observation 5 For all $j=1,2, \ldots, 14, g_{i} \sim g_{i} g_{j}^{-1}$.

We know that $i d \sim g_{j}^{-1}$. Let $g_{i}$ act on this line. Then $g_{i} \sim g_{i} g_{j}^{-1}$.

Observation 6 If $g$ is the unique element of order 3 and $g \in D$, then $g^{2} \in D$. If $g \notin D$, then 
$i d, g, g^{2}$ are not collinear.

If $g \in D$, then $g \sim i d$ on some $L$. Let $g$ act on $L$. Then $g^{2} \sim g$. Because $g^{2}=g^{-1}$ we have $g^{2} \in D$.

Suppose $g \notin D$, then clearly id $\nsim g$ and $i d \nsim g^{2}$. If $g \sim g^{2}$ then when $g$ acts on that line, we get $g^{2} \sim i d$ which is a contradiction. Thus no two of $i d, g, g^{2}$ are collinear.

Observation 7 There are points on $L$ with even order $\neq 2$.

Let $i$ be the unique element of order 2. Because this is a geometry, there is an associated strongly regular graph. For this graph $\mu=\alpha(t+1)=60$. Thus there are 60 points collinear with both $i$ and $i d$. These 60 points are thus in $D$. Let $g$ be one of these 60 points. If it has even order, then we are done. If $g$ has odd order, then we know that $i g$ is also one of the 60 points under consideration. Assume $g$ has order $2 k+1$. Then the lowest order possible for $i g$ is $2(2 k+1)$. This means $i g$ is a point of even order and because $i g$ is in $D$ it must be on some $L$. Therefore, there must be points on $L$ with even order.

Observation 8 If a point $g$ in $D$ has order 5, then either $g, g^{4} \in D$ or $g, g^{2}, g^{3}, g^{4} \in D$.

Suppose $g$ has order 5 and is in $D$. Then $g^{4}=g^{-1}$. Thus $g^{4} \in D$. Likewise $\left(g^{2}\right)^{-1}=g^{3}$. So if $g^{2} \in D, g^{3} \in D$. Thus either we have a complete graph for these five points, or just $g, g^{4}$ in $D$. 
Observation 9 If a point $g$ in $D$ has order 6 , then $g, g^{5}=g^{-1} \in D$. If the point of order 3 is in $D$, then $g^{2}, g^{4}$ are also in $D$.

Suppose $g$ has order 6 and is in $D$. Then $g^{5}=g^{-1}$. Thus $g^{5}$ is in $D$. If the unique point of order 3 is in $D$, then $\left(g^{2}\right)^{-1}=g^{4}$ so both $g^{2}$ and $g^{4}$ are in $D$.

It was hoped that just as in the $p g(5,5,2)$ case that a series of observations like these would either lead to a construction of a $p g(14,14,4)$ or to disproving its existence. However at this point we did not succeed and there is no conclusion yet as to whether $p g(14,14,4)$ does exist. Further investigation is necessary to make this determination. 



\section{Chapter 5}

\section{A Benson type theorem for strongly}

\section{regular graphs}

The discussion of the previous chapters has focused on the application of Benson's Theorem to partial geometries. However, the proof of Theorem 4 did not actually rely on the properties of the geometry, so now we return to the theorem and consider it as applied to a strongly regular graph. First we need to determine expressions for the eigenvalues in terms of the parameters of a strongly regular graph. 


\subsection{Eigenvalues for a strongly regular graph}

Recall from Lemma 3 that the eigenvalues of the adjacency matrix were the solutions to the equation

$$
\theta^{2}+(\mu-\lambda) \theta+(\mu-k)=0
$$

Previously we obtained specific expressions for these eigenvalues in terms of $s, t, \alpha$ as our focus was on partial geometries. Now we determine expressions for these eigenvalues in terms of the parameters for a strongly regular graph. As before, we know that one eigenvalue will be $k$. If we solve equation (5.1) in terms of $k, \mu, \lambda$ using the quadratic formula we obtain the two additional eigenvalues. Let $v_{1}, v_{2}, v_{3}$ represent the eigenvalues.

$$
\begin{gathered}
v_{1}=k \\
v_{2}=\frac{1}{2}\left(\lambda-\mu+\sqrt{(\lambda-\mu)^{2}+4(k-\mu)}\right) \\
v_{3}=\frac{1}{2}\left(\lambda-\mu-\sqrt{(\lambda-\mu)^{2}+4(k-\mu)}\right)
\end{gathered}
$$

We know that the sum of the multiplicities of the eigenvalues is $v$, and also that the sum of the eigenvalues is the trace of the adjacency matrix. If $m_{1}, m_{2}, m_{3}$ are the multiplicities, 
these conditions give us the following system of equations:

$$
\begin{gathered}
m_{1}+m_{2}+m_{3}=v \\
k+m_{2} v_{2}+m_{3} v_{3}=0
\end{gathered}
$$

To solve this system, we multiply the first equation by $v_{2}$ and subtract to get

$$
v_{2}-k+m_{3}\left(v_{2}-v_{3}\right)=v v_{2}
$$

Solving for $m_{3}$ gives us $m_{3}=\frac{k+v_{2}(v-1)}{v_{2}-v_{3}}$.

Substituting back in the first equation and solving for $m_{2}$ we get $m_{2}=\frac{-v_{3}(v-1)-k}{v_{2}-v_{3}}$.

Finally substituting for $v_{2}$ and $v_{3}$ gives the multiplicities in terms of $k, \lambda, \mu$.

$$
\begin{gathered}
m_{1}=1 \\
m_{2}=\frac{1}{2}\left(v-1-\frac{2 k+(v-1)(\lambda-\mu)}{\sqrt{(\lambda-\mu)^{2}+4(k-\mu)}}\right) \\
m_{3}=\frac{1}{2}\left(v-1+\frac{2 k+(v-1)(\lambda-\mu)}{\sqrt{(\lambda-\mu)^{2}+4(k-\mu)}}\right)
\end{gathered}
$$

In almost all cases, the eigenvalues are integers. The exception occurs in the case of the conference graph where $2 k-(v-1)(\lambda-\mu)=0$. In this graph $k=\frac{v-1}{2}, \lambda=\frac{v-5}{4}, \mu=$ $\frac{v-1}{4}$ which leads to $\sqrt{(\lambda-\mu)^{2}+4(k-\mu)}=\sqrt{v}$.

Automorphisms of graphs are much like those for geometries. However, in the case of 
graphs, edges are mapped to edges, while for a geometry, lines must be mapped to lines, the automorphisms for geometries are a subset of those for the accompanying strongly regular graph. In Figure 5.1 note that an automorphism of the geometry on the left requires that lines are mapped to lines. This means in the accompanying graph, that each set of a single color of edges must be mapped to another set of singly colored edges. In general, an automorphism of a graph simply requires that edges be mapped to edges.
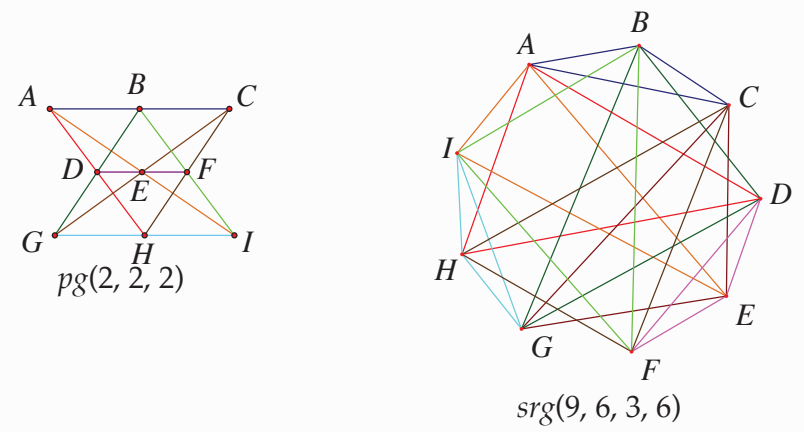

Figure 5.1: $p g(2,2,2)$ and its point graph

\subsection{The generalized theorem}

The conditions for Benson's Theorem in fact only require a strongly regular graph, so we can generalize this theorem to include automorphisms of strongly regular graphs as well. Suppose $\phi$ is an automorphism of the graph $G$ with order $n$. Then there is a $v \times v$ permutation matrix $P$ corresponding to this automorphism. In a strongly regular graph, $P A=A P[3]$ where $A$ is the adjacency matrix of the graph, and also $P^{n}=I$ where $I$ is the $v \times v$ identity matrix. Again we let $f$ represent the number of vertices fixed by the 
automorphism and $g$ be the number of vertices mapped to adjacent vertices by $\phi$. We can now state a generalization of Theorem 4 .

Theorem 18 Let $G$ be a strongly regular graph $\operatorname{srg}(v, k, \lambda, \mu)$ that is not a conference graph, and let $\phi$ be an automorphism of order $n$ of $G$. Let $\mu(d)$ denote the sum of the primitive $d^{\text {th }}$ roots of unity. Then for every integer $r$ and all divisors $d$ of $n$, there are nonnegative integers $a_{d}$ and $b_{d}$ such that

$$
k-r+\sum_{d \mid n} a_{d} \mu(d)\left(v_{2}-r\right)+\sum_{d \mid n} b_{d} \mu(d)\left(v_{3}-r\right)=-r f-g .
$$

As a consequence, the following congruence holds:

$$
k-v_{2} \equiv-v_{2} f+g\left(\bmod v_{3}-v_{2}\right) .
$$

Proof. Let $M$ be the matrix $M=A-r I$. Because $A$ has integer eigenvalues, so does M. These eigenvalues are $\tau_{0}=k-r, \tau_{1}=v_{2}-r$, and $\tau_{2}=v_{3}-r$ as subtracting $r$ from the diagonal elements of $A$ subtracts $r$ from each of the eigenvalues. The multiplicities are still as above, $m_{1}, m_{2}, m_{3}$. Because $P A=A P$, we note that $P M=M P$ and hence, $(P M)^{n}=P^{n} M^{n}=M^{n}$ as before. Likewise it again follows that the eigenvalues of $P M$ are the eigenvalues of $M$ multiplied by the appropriate $n^{\text {th }}$ roots of unity. The sum of each row of an adjacency matrix for a regular graph is $k$, so the sum of the elements of each row of $M$ is $k-r$. If $P M$ is multiplied by an all-one vector, $J$, the product will be a vector with each entry equal to $k-r$. In other words, $P M J=(k-r) J$. So $k-r$ is an eigenvalue for $P M$ 
with multiplicity $m_{1}=1$. Now let $d$ be a (positive) divisor of $n$, and let $\xi_{d}$ be a primitive $d^{\text {th }}$ root of unity. The eigenvalues of $M$ are integers. Thus the eigenvalues of $P M$ are these integers multiplied by roots of unity. By the same Lemma noted in Theorem 4, each of $\xi_{d}\left(v_{2}-r\right)$ will have the same multiplicity, dependent only on $d$. Denote this multiplicity by $a_{d}$. In the same way the multiplicity of the eigenvalue $\xi_{d}\left(v_{3}-r\right)$ will only depend on $d$. Denote this multiplicity by $b_{d}$. The sum of the primitive $d^{\text {th }}$ roots of unity is $\mu(d)$. We now obtain an equation similar to that in Theorem 4 .

$$
\operatorname{trace}(P M)=k-r+\sum_{d \mid n} a_{d} \mu(d)\left(v_{2}-r\right)+\sum_{d \mid n} b_{d} \mu(d)\left(v_{3}-r\right)
$$

On the other hand, the trace of $P M$ must equal $-r f+g$ as each entry on the diagonal of $P M$ is $-r$ if the corresponding vertex is fixed and 1 if the corresponding vertex is mapped to a collinear vertex by the automorphism. Therefore, we get

$$
k-r+\sum_{d \mid n} a_{d} \mu(d)\left(v_{2}-r\right)+\sum_{d \mid n} b_{d} \mu(d)\left(v_{3}-r\right)=-r f+g
$$

Setting $r=v_{2}$ we obtain

$$
k-v_{2} \equiv-v_{2} f+g\left(\bmod v_{3}-v_{2}\right)
$$

proving the theorem.

We can also use this result to obtain expressions for the multiplicities of the eigenvalues. 
When $\phi$ is an automorphism of prime order, $p$ of $G$, setting $r=v_{3}$ we obtain the equation $k-v_{3}+\sum_{d \mid p} a_{d} \mu(d)\left(v_{2}-v_{3}\right)=-v_{3} f+g$. Because $p$ is prime, we have $\mu(p)=-1$ and $\mu(1)=1$. Also we know that $a_{1}+a_{p}=m_{2}$. These observations give us the following system of equations in $a_{1}$ and $a_{p}$ :

$$
\left\{\begin{array}{c}
a_{1}\left(v_{2}-v_{3}\right)-a_{p}\left(v_{2}-v_{3}\right)=-v_{3}(f-1)+g-k \\
a_{1}+(p-1) a_{p}=m_{2}
\end{array}\right.
$$

Multiplying the second equation by $\left(v_{2}-v_{3}\right)$ and subtracting from the first equation gives

us

$$
-a_{p} p\left(v_{2}-v_{3}\right)=-v_{3}(f-1)+g-k-m_{2}\left(v_{2}-v_{3}\right)
$$

Solving for $a_{p}$ we get $a_{p}=\frac{v_{3}(f-1)-g+k+m_{2}\left(v_{2}-v_{3}\right)}{p\left(v_{2}-v_{3}\right)}$.

Then substituting the expressions in terms of $k, v, \lambda, \mu$ this gives us

$$
a_{p}=\frac{-2 g+(f-v)\left(\lambda-\mu-\sqrt{(\lambda-\mu)^{2}+4(k-\mu)}\right)}{2 p \sqrt{(\lambda-\mu)^{2}+4(k-\mu)}} .
$$

Now $a_{1}=m_{2}-a_{p}(p-1)$, so we obtain the following expression for $a_{1}$.

$$
a_{1}=\frac{2 g(p-1)-2 p k-(f(p-1)+v-p)\left(\lambda-\mu-\sqrt{(\lambda-\mu)^{2}+4(k-\mu)}\right)}{2 p \sqrt{(\lambda-\mu)^{2}+4(k-\mu)}}
$$

These values should be nonnegative integers so calculating these values can give insight as to whether or not specific strongly regular graphs admit certain automorphisms. 


\section{Chapter 6}

\section{Summary and future work}

The major results of this thesis can be stated as follows:

- In Chapter 2 we developed expressions for the multiplicities of the eigenvalues of the matrix $M=A+(t+1) I$ where $A$ is the adjacency matrix of the point graph of a partial geometry when that partial geometry is acted upon by an automorphism of prime order.

Theorem 5 Given a partial geometry $\operatorname{pg}(s, t, \alpha)$ acted upon by an automorphism of prime order, $n$, the matrix $Q M$ as defined in the proof of Theorem 4 has eigenvalues with multiplicities $a_{1}$ and $a_{2}$ as follows:

$$
\begin{aligned}
& a_{1}=\frac{\alpha(n-1)((t+1) f+g)+(s+1)(t+1)(s t-\alpha(n-1))}{\alpha n(s+t+1-\alpha)} \\
& a_{2}=\frac{(s+1)(t+1)(s t+\alpha)-\alpha((t+1) f+g)}{\alpha n(s+t+1-\alpha)}
\end{aligned}
$$


- In Chapter 3 we developed necessary conditions for the existence of a generalized maximal arc.

Theorem 9 If $\mathcal{S}(\mathcal{M})$ is constructed from $P G(m, q)$ 's in $P G(n, q)$ where $q$ is a power of some prime, $p$, then $n=2 m+1+\frac{m+1}{r+1}$ for some $r$, a non-negative integer, and $\alpha=\frac{\left(p^{k}-1\right)\left(q^{m+1}-1\right)}{q^{n-2 m-1}-1}$ where $0<p^{k}<q^{n-2 m-1}$.

- In Chapter 4 we found a list of potential rigid-type partial geometries.

\section{Table 4.1}

Values of $s, t, \alpha$ for potential partial geometries.

\begin{tabular}{|c|c|c|c|c|c|c|c}
$s$ & $t$ & $\alpha$ & $x$ & $v$ & $k$ & $\lambda$ & $\mu$ \\
\hline 5 & 5 & 2 & 1 & $81=3^{4}$ & 30 & 9 & 12 \\
\hline 11 & 23 & 3 & 2 & $1024=2^{10}$ & 264 & 56 & 72 \\
\hline 14 & 14 & 4 & 1 & $750=2 \cdot 3 \cdot 5^{3}$ & 210 & 55 & 60 \\
\hline 19 & 59 & 4 & 3 & $5625=3^{2} \cdot 5^{4}$ & 1140 & 195 & 240 \\
\hline 29 & 119 & 5 & 4 & $20736=2^{8} \cdot 3^{4}$ & 3480 & 504 & 600 \\
\hline 27 & 27 & 6 & 1 & $3430=2 \cdot 5 \cdot 7^{3}$ & 756 & 161 & 168 \\
\hline 34 & 69 & 6 & 2 & $13720=2^{3} \cdot 5 \cdot 7^{3}$ & 2380 & 378 & 420 \\
\hline 41 & 209 & 6 & 5 & $60025=5^{2} \cdot 7^{4}$ & 8610 & 1085 & 1260 \\
\hline 55 & 335 & 7 & 6 & $147456=2^{14} \cdot 3^{2}$ & 18480 & 2064 & 2352 \\
\hline 44 & 44 & 8 & 1 & $10935=3^{7} \cdot 5$ & 1980 & 351 & 360 \\
\hline 62 & 188 & 8 & 3 & $91854=2 \cdot 3^{8} \cdot 7$ & 11718 & 1377 & 1512 \\
\hline 71 & 503 & 8 & 7 & $321489=3^{8} \cdot 7^{2}$ & 35784 & 3591 & 4032
\end{tabular}


- In Chapter 5 we proved a Benson type theorem for strongly regular graphs.

Theorem 18 Let $G$ be a strongly regular graph $\operatorname{srg}(v, k, \lambda, \mu)$ that is not a conference graph, and let $\phi$ be an automorphism of order $n$ of $G$. Let $\mu(d)$ denote the sum of the primitive $d^{\text {th }}$ roots of unity. Then for every integer $r$ and all divisors $d$ of $n$, there are nonnegative integers $a_{d}$ and $b_{d}$ such that

$$
k-r+\sum_{d \mid n} a_{d} \mu(d)\left(v_{2}-r\right)+\sum_{d \mid n} b_{d} \mu(d)\left(v_{3}-r\right)=-r f-g
$$

As a consequence, the following congruence holds:

$$
k-v_{2} \equiv-v_{2} f+g\left(\bmod v_{3}-v_{2}\right) .
$$

In the future I would like to continue to investigate some of the potential rigid-type geometries listed in Chapter 4 beginning with more work on the potential $p g(14,14,4)$. A determination as to the existence of this partial geometry could provide insight into the infinite family of potential rigid-type partial geometries with $s=t$. I would also like to continue to work on ways to bring material of this nature down to students at the pre-college level. The adventure of discovering mathematics is a grand one and I hope to impart some of the joy to younger students, encouraging them to pursue this adventure in their future. 


\section{References}

[1] A. Blokhuis, and S. Ball, An easier proof of the maximal arcs conjecture, Proceedings of the American Mathematical Society 126 (1998), no. 11, 3377-3380.

[2] C. T. Benson, On the structure of generalized quadrangles, J. Algebra 15 (1970), 443-454.

[3] N. Biggs, Algebraic Graph Theory, Cambridge University Press. (1993), 116.

[4] R. C. Bose, Strongly regular graphs, partial geometries and partially balanced designs, Pacific J. Math. 13 (1963), 389-419.

[5] F. De Clerck, Constructions and Characterizations of (Semi) partial Geometries, Summer School on Finite Geometries, Potenza 2 -13 September 1997.

[6] F. De Clerck, M. Delanote, N. Hamilton, and R. Mathon, Perp-systems and partial geometries, Advances in Geometry 2 (2001), 1-12.

[7] R. H. F. Denniston, Some maximal arcs in finite projective planes, Journal of Combinatorial Theory, Volume 6, Issue 3, (1969), 317-319. 
[8] S. De Winter, Constructions, characterizations and classifications of SPG-reguli, SPG-systems and the related semipartial geometries, Diss. Ph.D.-Thesis, Ghent University, (2004), 6.

[9] S. De Winter and K. Thas, "Generalized quadrangles with an abelian Singer group," Des Codes Cryptogr. 39(1) (2006), 81-87.

[10] S. De Winter, Partial geometries $\operatorname{pg}(s, t, 2)$ with an abelian Singer group and a characterization of the vanLint-Schrijver partial geometry, J. Algebraic Combin. $\mathbf{2 4}$ (2006), no. 3, 285-297.

[11] M. Gockenbach, Finite Dimensional Linear Algebra, CRC Press, (2010), 9, 426, 240, 232-233.

[12] D. Kreher, Combinatorics and Graph Theory, course materials for MA4209, Michigan Technological University (2011), 35.

[13] K. H. Leung, S. L. Ma, and B. Schmidt, Proper partial geometries with Singer groups and pseudogeometric partial difference sets, Journal of Combinatorial Theory, Series A 115.1 (2008), 147-177.

[14] J. Singer, A Theorem in Finite Projective Geometry and Some Applications to Number Theory, Transactions of the American Mathematical Society, Vol. 43, No. 3 (May, 1938), 377-385. 
[15] Temmermans, Beukje, Dualities and Collineations of Projective and Polar spaces and of Related Geometries, Diss. Ph. D.-Thesis, Ghent University, (2010).

[16] J. A. Thas, Construction of partial geometries, Simon Stevin 46 (1973), 95 - 98.

[17] J. A. Thas, Construction of maximal arcs and partial geometries, Geometriae Dedicata, Volume 3, Issue 1, (1974), 61 - 64. 


\section{Appendix A}

\section{Partial geometries and strongly regular graphs - an enrichment unit for high school}

The following document is a four lesson unit to be used as an enrichment to the standard curriculum. In the typical mathematics sequence students have little exposure to finite geometries. Proof is often limited to the two-column format and students are rarely asked to first create a conjecture before proving it. By providing an opportunity to explore a new topic and make conjectures, this unit aims to broaden the student's horizons as to what mathematics is and how it is done. The unit begins with a summary of mathematics involved. This includes a discussion of geometry in general along with information on partial geometries and strongly regular graphs. Following this introductory material, each lesson consists of information and a lesson plan for the teacher, a worksheet of examples and exercises for the student, and solutions for the exercises. The exercises are designed to be done in groups, and culminate in the proof of the relationship between the parameters of a partial geometry and its strongly regular graph. 


\section{Introduction and Background Mathematics}

In the most general sense, geometry is the branch of mathematics dealing with properties and relationships between points, lines, and figures in space according to a specific set of assumptions. In school, the geometry studied is generally that of Euclid, where there are infinitely many points on each line, and infinitely many lines. However, it is possible to change the basic assumptions, and limit the system to a finite number of points and lines. Working with a finite set of points and lines provides an opportunity for students to conjecture and develop proof skills in a different setting. Extending these ideas beyond the typical models of points and lines to include algebraic objects such as variables and graphs also provides an opportunity to generalize and see the power of mathematics. Geometries consisting of sets of objects referred to as points and lines along with an incidence relation between them are called point line geometries. Every geometry has a set of axioms, or postulates, from which theorems and properties are deduced. The familiar Euclidean geometry rests on five such postulates:

1. Any two points define a line segment.

2. Any line segment can be extended indefinitely to be a line.

3. Given any line segment a circle can be constructed having the segment as its radius and one endpoint as its center.

4. All right angles are congruent.

5. If two lines intersect a third (called a transversal) so that the sum of the interior angles on one side of the third line is less than two right angles, then the original two lines intersect each other on that side.

The fifth postulate is equivalent to the statement that given a line and a point not on that line there is exactly one line parallel to the given line through the given point. However, it is possible to create a geometry with a different set of axioms. Many such systems exist including the non-Euclidean geometries that accept the first four postulates and contradict the fifth by either allowing for an infinite number of parallels through a point or allowing none. If we limit ourselves to a finite point-line geometry, we can create systems with a simpler set of axioms. For instance, the following postulates define a partial geometry in terms of three parameters, $s, t$, and $\alpha$.

1. There are $s+1$ points on every line.

2. There are $t+1$ lines incident with every point.

3. Through any two points there is at most one line. 
4. Given a line and a point not on that line, there are $\alpha$ points on the line that are collinear with the given point.

Because the three values, $s, t, \alpha$, describe the partial geometry we refer to a specific partial geometry as $p g(s, t, \alpha)$, substituting specific values for each partial geometry.

While it is not necessary or even practical to create diagrams of all partial geometries, it is helpful to look at some simple examples. For instance a triangle is a representation of $p g(1,1,2)$. There are $1+1=2$ points on each line, $1+1=2$ lines through each point, and each point not on a line is collinear with 2 points on that line. Notice that point $A$ is collinear with $B$ and $C$, so $\alpha$ is 2 .

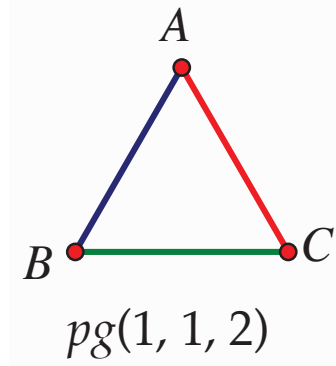

A simple rectangle is also representation of a partial geometry, $p g(1,1,1)$. If we include the diagonals, then the we have $p g(1,2,2)$.
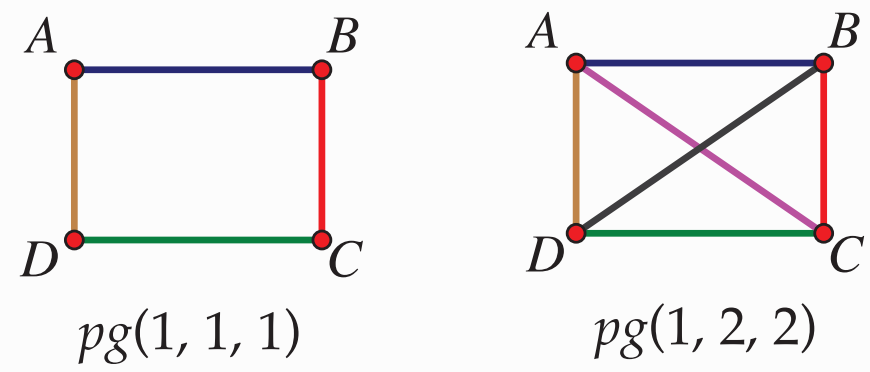

None of these is the representation of a proper partial geometry however, because to be proper, $\alpha$ must be less than both $s$ and $t$. The next figure is called a generalized quadrangle and it is a representation of the proper partial geometry $p g(2,2,1)$. In this figure, each colored arc or line segment represents a line. Note that there are $2+1=3$ points on each line. Only the points marked as such are actually points. Other apparent intersections do not represent points of the geometry. Because visual representations of most proper partial geometries become rather complex, in this unit most of the geometries students will work with will not be proper. 


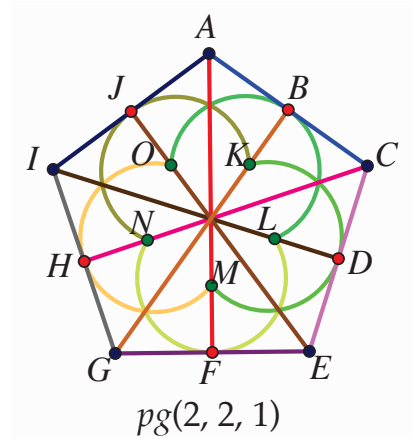

Graphs are one tool used to provide insight into a geometry. In this context a graph is a collection of vertices and edges, not the familiar Cartesian graph of a function. Each vertex represents a point in the geometry. The edges of the graph indicate collinearity. Every pair of collinear points is connected by an edge. If two vertices are connected by an edge, they are called adjacent. The graph below has four vertices and six edges.

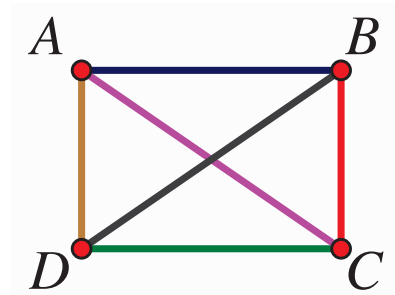

Each vertex of a graph has a degree associated with it. The degree is the number of edges that meet at that vertex. In the graph above, each vertex has degree three. When all vertices have the same degree, the graph is called regular. The graph above is an example of a 3-regular graph because each vertex has degree three.

Simple polygons, such as a rectangle or triangle, are examples of 2-regular graphs, so 2-regular graphs are possible for all numbers of vertices. However, it is not possible to create all values of regular graphs for every number of vertices. For $n$ vertices to have a $k$-regular graph, the value of $n k$ must be even. This is because $n k$ counts $k$ edges at each of $n$ vertices, thus counting each edge twice. When there is an odd number of vertices, the only $k$-regular graphs are thus only those where $k$ is even.

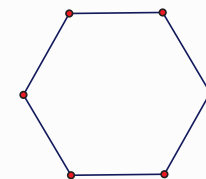

2-regular

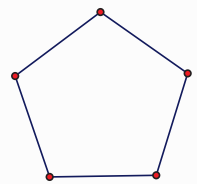

2-regular

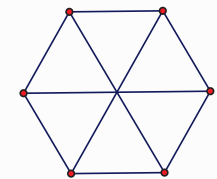

3-regular

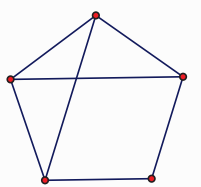

3-regular is not possible

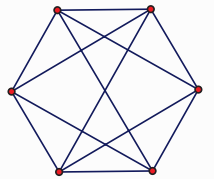

4-regular

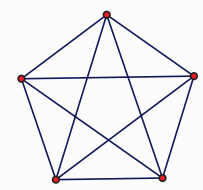

4-regular

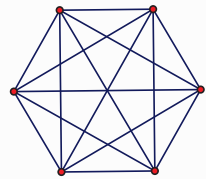

5-regular 
There is a regular graph associated with every partial geometry. When there are only two points on each line, the graph and geometry look the same. However, when there are more than two points on each line the graph and the geometry are visually different. Suppose there are three points on each line. That means that in the graph these three points must all be adjacent to each other. That is, they form a triangle in the graph. The graph for the generalized quadrangle shows this. In the geometry, the points $A, B, C$ were collinear. In the graph notice that they form a triangle. Every other set of three collinear points in the geometry corresponds to a similarly colored triangle in the graph.

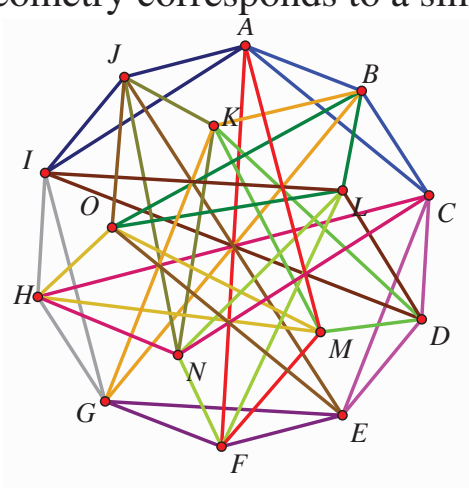

For a somewhat simpler example of this consider the following geometry and its graph.
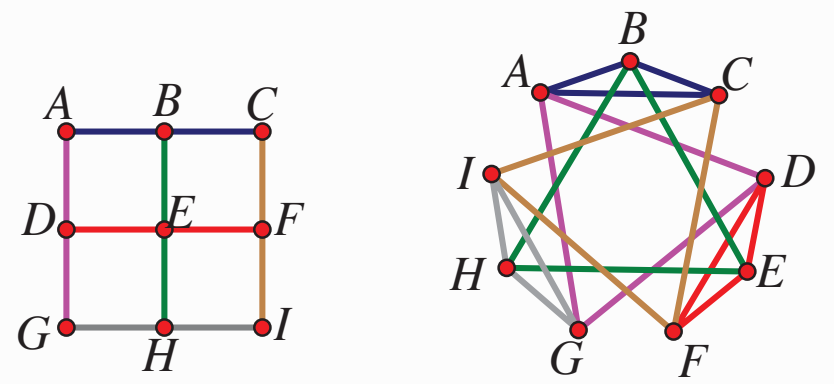

Some regular graphs are also classified as strongly regular. This implies further conditions. First, any two adjacent vertices, or vertices connected by an edge, are commonly adjacent to the same number of vertices. Similarly, any two non-adjacent vertices will always have the same number of common neighbors. The graph, $\operatorname{srg}(v, k, \lambda, \mu)$, is defined by four parameters,

- $v$ : number of vertices

- $k$ : degree of graph

- $\lambda$ (lambda): number common neighbors of adjacent vertices

- $\mu(\mathrm{mu})$ : number of common neighbors of non-adjacent vertices

For example, in the graph below, any two adjacent vertices are both adjacent to two other vertices. For $A$ and $B$ these common neighbors are $C$ and $D$. While two non-adjacent 
vertices such as $A$ and $F$ have four common neighbors, $B, C, D$, and $E$.

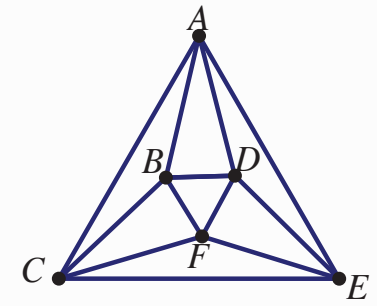

$\operatorname{srg}(6,4,2,4) \quad$ Each partial geometry has an accompanying strongly regular graph. However, only some strongly regular graphs are related to partial geometries. The parameter values for a partial geometry and its associated strongly regular graph are closely related. In the final lesson of this unit students will prove the theorem:

Theorem 19 The point graph of a pg $(s, t, \alpha)$ is a strongly regular graph with parameters $(v, k, \lambda, \mu)=\left(\left(\frac{s t+\alpha}{\alpha}\right), s(t+1), s-1+t(\alpha-1), \alpha(t+1)\right)$.

The proof is not trivial. Students will use diagrams to help in their reasoning as they prove each part of this relationship. Many students do not have much experience writing paragraph proofs, but in future mathematics courses, most work is done in an expository fashion. Each section of the proof makes use of diagrams to aid in reasoning. This is also a technique that is often used as a mathematician seeks new understanding. Thus the experience of working through this major proof provides insight into future mathematical techniques. 


\section{Lesson 1: What is a Geometry?}

Begin this lesson with a discussion by asking "What is geometry?" Guide the discussion to create a definition of geometry that is the most basic possible: a study of points, lines, and the relationships between them. All of the other things mentioned, such as triangles, congruence, circles, proof, are simply expansions of the relationships between points and lines. Then ask "What is a point?" Here students will probably offer up the standard image of an infinitely small "dot" or location. Suggest the possibility of a point as an idea, that other things can represent points other than dots. Recall that a point is an undefined term in Euclidean geometry. Anything can be used to represent a point, a dot, a letter, a number, a person. Finally ask, "What is a line?" By now students may have the idea that you want them to think more broadly. They may offer more creative interpretations. Be open to all ideas. Based on what we use to represent a point, this can help determine what represents a line. For instance, if people represent points, then the relationship of friends on Facebook might represent a line. Another example is to think of rooms as points and doors between rooms are lines connecting the points. The idea is to get the students to begin to think more abstractly about the concepts of points and lines. In this lesson, points and lines will be represented in many ways, and the idea of incidence will also be defined accordingly. They will be working with a finite number of points and must remember that any two points can only be on one line. Work through the following examples as a class.

\section{Example 1}

What is the smallest number of points you need to make 3 lines with 2 points on every line? Can you represent your answer in more than one way?

Let students work on this in groups for a few minutes and then present solutions. Look for the smallest number of points, that is, three. The solution can be represented as a triangle. If no one has another representation, suggest the following idea. Let the points be represented by $a, b, c$. The lines are the combinations of two letters, $a b, a c, b c$. Using this representation, pick a point, $a$, and a line not containing $a$, that is, $b c$. How many of the points on $b c$ are collinear with $a$ ? Students should readily see that both points on $b c$ are collinear with $a$. Is this true if you pick any point and line not containing that point?

\section{Example 2}

What is the smallest number of points you need so that each point is on two lines, each line contains two points, and for any point and line not containing that point, only one point is collinear with the given point? Try and make both a picture and a letter representation 
of this situation. If students are puzzled as to how to begin, suggest they try to satisfy the conditions with three points. If they cannot, then add a fourth point and try again. Keep adding one point at a time until you are able to meet the conditions.

This should be a rectangle for the visual representation. Using letters, if the points are $a, b, c, d$, then the lines are $a b, b c, c d, d a$. Students should then complete the remaining exercises in small groups. Share answers with the rest of the class as time permits. 


\section{What is a Geometry?}

\section{Example 1}

What is the smallest number of points you need to make 3 lines with 2 points on every line? Can you represent your answer in more than one way?

\section{Example 2}

What is the smallest number of points you need so that each point is on two lines, each line contains two points, and for any point and line not containing that point, only one point is collinear with the given point? Try and make both a picture and a letter representation of this situation.

Picture

Letters 


\section{In Your Group}

1. Create both a picture representation and a letter representation of a geometry where each point is on three lines, there are two points on each line, and for any point and line not containing that point, two points are collinear with the given point. (Hint: you can do this with four points.)

2. Another way to create a geometry is to use a table like the one below. Notice that the table is symmetric across the empty diagonal. Each box above the diagonal contains a unique letter.

\begin{tabular}{|l|l|l|l|}
\hline & $A$ & $B$ & $C$ \\
\hline$A$ & & $D$ & $E$ \\
\hline$B$ & $D$ & & $F$ \\
\hline$C$ & $E$ & $F$ & \\
\hline
\end{tabular}

Here each of the rows represents a line and each letter represents a point. So there are four lines, each with three points. Create a geometry using a table with five rows and five columns.

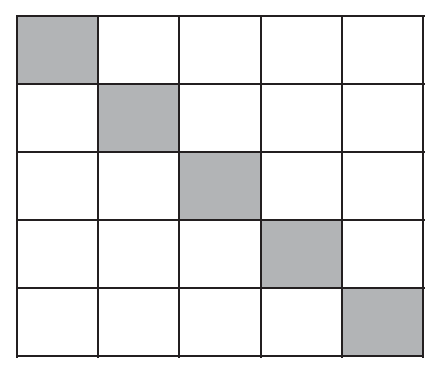

(a) List each of the lines using the letters. 
(b) Choose any point and a line that does not contain that point. How many points on the line are collinear with the chosen point.

(c) Creating a visual representation of the geometry you just created is rather tricky so instead, try to create a visual representation of the 6-point geometry in the given table above. Keep in mind that one of the "lines" may need to curve in order to make the geometry complete.

3. Create a letter representation of the geometry that has three points on every line, two lines through every point, and for any point and line not containing that point, only one point is collinear with the given point. Do not try to make a drawing of this system, just create a letter representation. (Hint: you only need 9 points and 6 lines.) 


\section{Lesson 1 answers}

\section{In your group}

1. Here each line is shown in a different color.

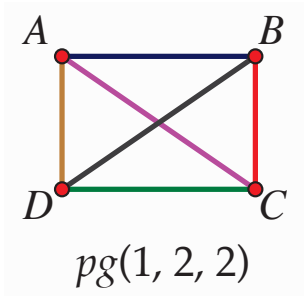

2. The table is shown below.

\begin{tabular}{|l|l|l|l|l|}
\hline & $A$ & $B$ & $C$ & $D$ \\
\hline$A$ & & $E$ & $F$ & $G$ \\
\hline$B$ & $E$ & & $H$ & $I$ \\
\hline$C$ & $F$ & $H$ & & $J$ \\
\hline$D$ & $G$ & $I$ & $J$ & \\
\hline
\end{tabular}

(a) lines: $A B C D, A E F G, B E H I, C F H J, D G I J$

(b) There are two points that are collinear. For instance, $A$ is not on line $B E H I$, but $A$ and $B$ are collinear as are $A$ and $E$.

(c) The geometries for both the $4 \times 4$ and $5 \times 5$ grid are shown below:
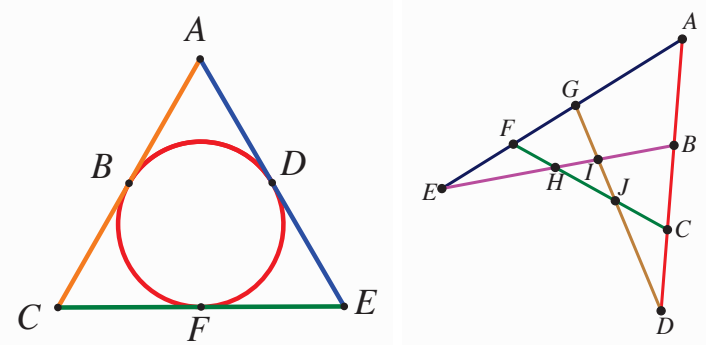

3. One representation might consist of these lines: abc, def, ghi, adg, beh,cfi. While the problem did not ask for a figure, one is given below.

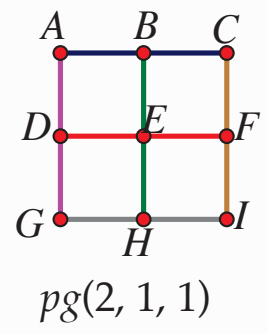




\section{Lesson 2: Graphs}

Introduce the lesson by discussing the fact that each of the geometries discussed so far also can be represented by a graph. This is not like the graph of a function, but a different sort of graph. Display a graph similar to the one below for the purposes of defining the vocabulary associated with a graph. When a line has only two points, the graph is the same as the geometry. However, when there are more points on a line, the graph will look very different from the visual representation of the geometry. Use the geometry from exercise 3 in the previous lesson to illustrate this. Use a set of letters from one of the groups. Look the lines that contain the letter $A$. In a picture of the geometry, you would see two lines. But on the graph, you will need to have an edge going from $A$ to each of the other points on those lines. Ask students to determine how many vertices will be connected, or adjacent, to $A$ on the graph. They should see that four points will be adjacent to $A$. Then go on to the examples to introduce the idea of degree.

\section{Example 1}

A graph consists of a set of edges and vertices. This graph has six vertices and ten edges. The number of edges that meet at a vertex is called the degree of that vertex. The vertex $A$ has degree two while vertex $D$ has degree four. Have students name the degrees of the remaining vertices.

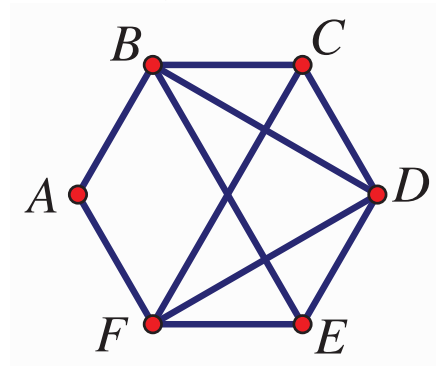

If every vertex has the same degree, the graph is said to be regular. Have students try to turn this graph into a regular graph by adding edges. A possible solution is shown below.

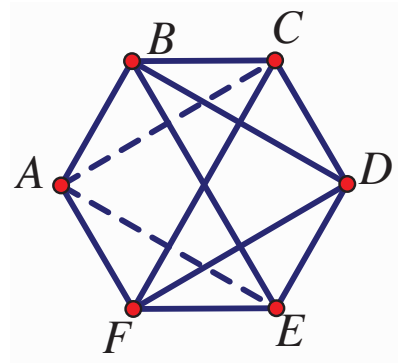

This is now a regular graph of degree four. More edges can also be added to make the graph regular with degree five. 


\section{Example 2}

Graphs can be drawn on any number of vertices. Is it possible to draw a regular graph using two vertices? What degree is each vertex? What types of regular graphs can you draw using three vertices? What if you use four vertices? Let students experiment and introduce the terminology of $k$-regular graphs. Students should discover that with two vertices you can make a 1-regular graph while with three vertices you can only make a 2-regular graph. Using four vertices they should be able to make both a 2-regular graph and a 3-regular graph.

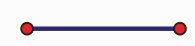

1-regular

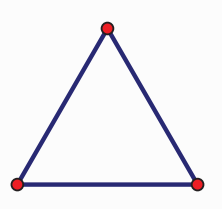

2-regular

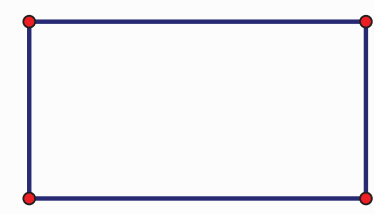

2-regular

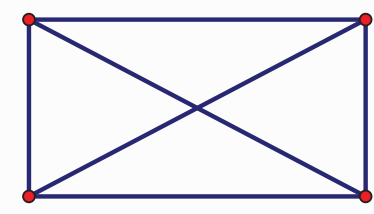

3-regular

In the exercises students will investigate the relationship between the number of vertices and the possible regular graphs. They will also discover that a partial geometry has a regular graph and begin to investigate the connection between the degree of the regular graph and the parameters of the geometry. 


\section{Lesson 2: What is a Graph?}

\section{Example 1}

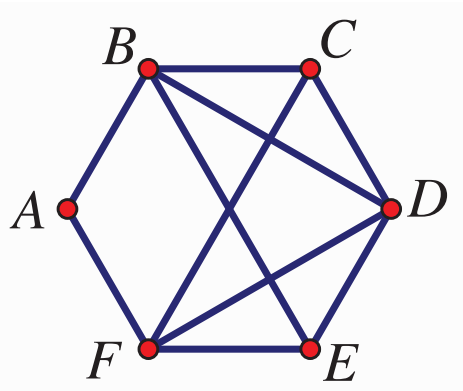

What is the degree of each vertex in the graph shown?

Add edges to the graph to make it regular. What is its degree now?

\section{Example 2}

Graphs can be drawn on any number of vertices.

Is it possible to draw a regular graph using two vertices? What degree is each vertex?

Draw all of the regular graphs using three vertices. What degree is each vertex?

Draw all of the regular graphs using four vertices. What are the degrees of each vetex? 


\section{In your group}

1. In Example 2 you considered regular graphs on 2, 3, and 4 vertices. What happens if you have more vertices?

(a) Using five vertices draw an example of each type of regular graph possible.

(b) Repeat for six vertices.

(c) What types of regular graphs are possible for seven vertices? Explain.

(d) What types of regular graphs are possible for eight vertices? Explain.

(e) What types of regular graphs are possible for $n$ vertices? Explain. 
2. Every geometry has an associated graph. In the visual representation of the geometry, all points on a line are shown on the same line. When you create the graph, all points that are on a line in the geometry are adjacent in the graph.

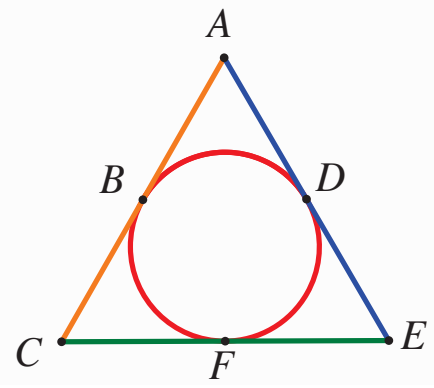

(a) In the geometry above $A, B$, and $C$ are on a line. In the graph $A$ will be adjacent to $B$ and also to $C$. What other points will be adjacent to $A$ in the graph?

(b) Draw the graph associated with this geometry.

(c) Is the graph regular? If so, what is its degree?

3. Below is a visual representation of another geometry. Will the associated graph be regular? If so, what is its degree? Explain how how you determined this value.

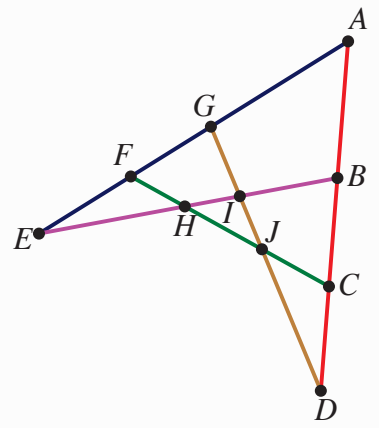




\section{Lesson 2 Answers}

\section{In your group}

1. (a) Regular graphs on five vertices:

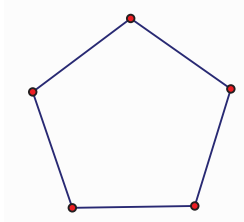

2-regular

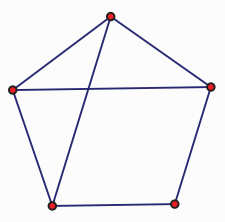

3-regular is not possible

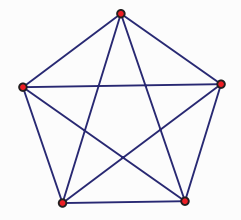

4-regular

(b) Regular graphs on six vertices:

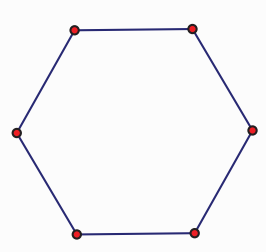

2-regular

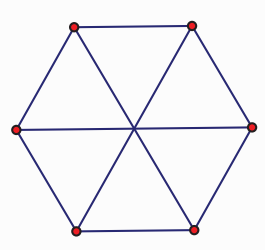

3-regular

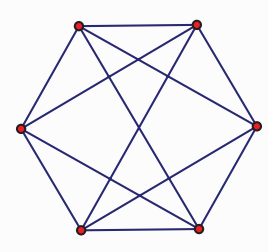

4-regular

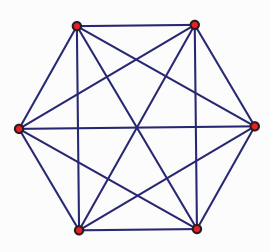

5-regular

(c) On seven vertices the only regular graphs are 2-regular, 4-regular, and 6-regular.

(d) On eight vertices all regular graphs from 2-regular through 7-regular are possible.

(e) On $n$ vertices all regular graphs from 2-regular through $(n-1)$-regular are possible when $n$ is even. When $n$ is odd, only the even valued regular graphs are possible. Suppose $n$ is odd and you want to make a 3-regular graph. Then each vertex must have 3 edges meeting at it. This makes a total of $3 n$ edges. But because each edge touches two vertices, the total of $3 n$ counts each edge twice. So $3 n$ must be an even number. However, since $n$ was also odd, that means $3 n$ is odd. This is a contradiction. Thus it is impossible to have a 3-regular graph on an odd number of vertices. The same problem occurs whenever you try to make an odd-regular graph on an odd number of vertices. 
2. (a) $A$ will also be adjacent to $D$ and $E$.

(b) Two possible graphs are shown below.
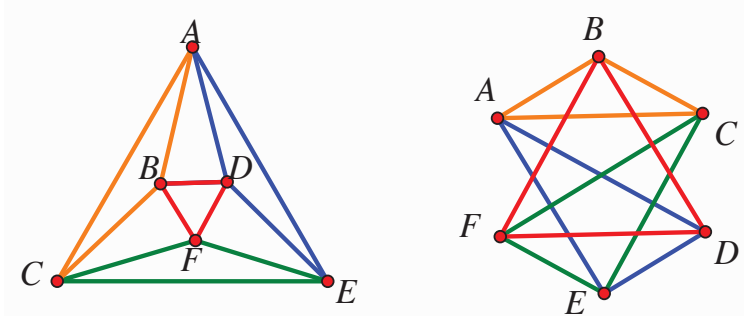

(c) The graph is 4-regular.

3. The graph will be 6-regular. Consider a point such as $A$. It is collinear with six other points. So it will be adjacent to six points in the graph. Because this is true for any point, it means the graph will be 6-regular. Another way to consider this is as follows: There are four points on each line so any point will be adjacent to three other points from that line. There are two lines through each point so a given point will be adjacent to three points on each of two lines, making the vertex degree six and the graph is 6-regular. 


\section{Strongly Regular Graphs}

In this lesson students will look more closely at properties of some regular graphs and begin to make connections between graphs and geometries. The specific type of regular graphs that we will focus on are called strongly regular graphs. These graphs are regular but are not complete. A complete graph contains all possible edges so that every vertex is adjacent to every other vertex. Strongly regular graphs have two additional parameters $\lambda$ (lambda), the number of common neighbors to two adjacent vertices, and $\mu$ (mu), the number of common neighbors to two non-adjacent vertices. Complete graphs are excluded from this discussion as all vertices are adjacent and it makes no sense to talk about a value of $\mu$ for them. The values of $v$ (number of vertices), $k$ (degree), $\lambda$ and $\mu$ in the graph associated with a geometry are determined by the parameters of the geometry $(s, t, \alpha)$. In this lesson students will hypothesize the relationship for the values of $k$ and $\mu$. The relationships for $v$ and $\lambda$ are more complicated and students verify these relationships in the examples. In Lesson 4 students will prove their conjectures.

Introduce the lesson by presenting the graph from example 1 in the previous lesson.

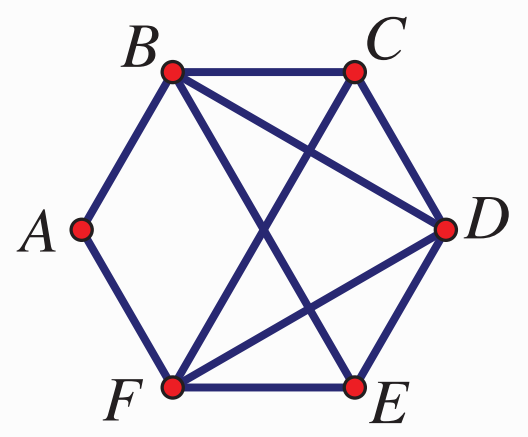

Now consider two adjacent vertices in this graph, for example $B$ and $C$. Ask "how many vertices are adjacent to both of these vertices?" Only vertex $D$ is adjacent to both. We say that $B$ and $C$ have one common neighbor. Ask "how many common neighbors do $D$ and $F$ have?" In this case they have two, $C$ and $E$. Next look at two non-adjacent vertices such as $A$ and $D$. Ask "how many common neighbors do these vertices have?" Two, vertex $B$ and $F$. Now move on to considering regular graphs. Start with a very basic graph such as a rectangle. Identify the number of vertices and the degree. Now determine the number of common neighbors to any two adjacent vertices. In this case the value is zero. Also determine the number of common neighbors to any two nonadjacent vertices to be one.

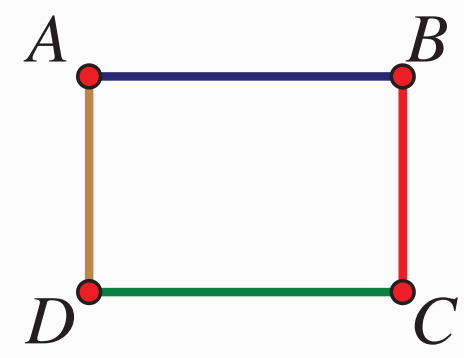




\section{Example 1}
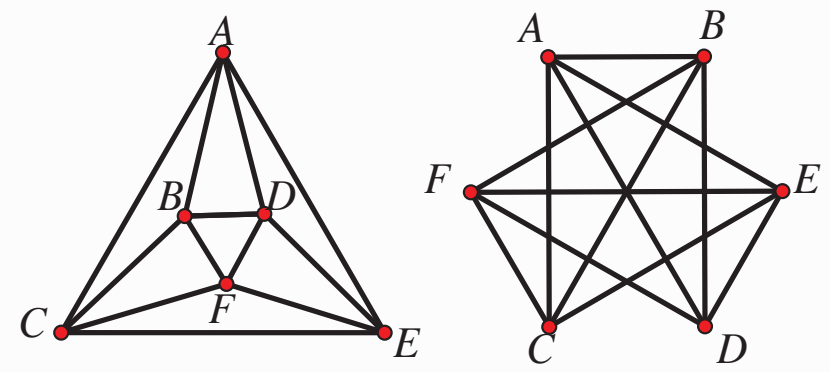

Present these graphs and have students determine the values of the four parameters $(v, k, \lambda, \mu)$. Answer: $(6,4,2,4)$. Then discuss how this is actually the same graph. If you have Geometers Sketchpad or similar software, consider creating the first graph and then letting students manipulate it into the second form. Uncovering the geometry from the graph is not possible without making some assumptions. So assume $A B C$ is a line in the geometry. What are the other lines? Each triangle in the figure might be considered a line, but remember, any two points can be on at most one line. So $A B D$ cannot be a line since $\mathrm{A}$ and $\mathrm{B}$ are already on one line. The remaining lines are $A D E, B D F$, and $C E F$. Try making a different initial assumption such as $A B D$ is a line. Ask "What are the other lines now?" Students should find the other lines to be $B C F, D E F$, and $A C E$. This last one may be a bit more difficult to identify.

\section{Example 2}

Every partial geometry can be described by three parameters. They refer to the number of points on each line, the number of lines incident to each point, and the number of points on a line that are collinear with a point not on the line. Consider the geometry pictured here.

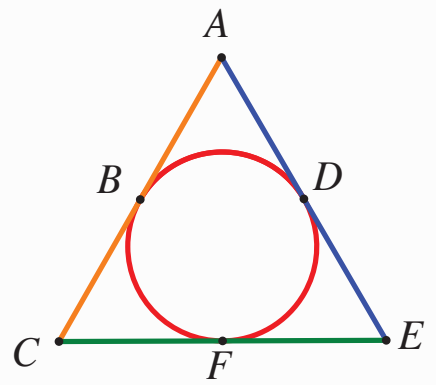

This is referred to as $p g(2,1,2)$. The first value is $s$. There are $s+1$ points on each line. The second value is $t$. There are $t+1$ lines through every point. The final value is $\alpha$. There are 2 points on each line that are collinear with any point not on the line. This graph associated with this partial geometry is the one in Example 1. What are the values of $s, t$, and $\alpha$ for 
the geometry represented by this figure?

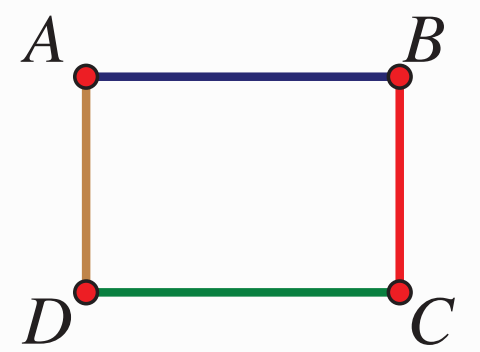

Answer $p g(1,1,1)$. Be careful to remember that there are $s+1$ points on a line, not $s$. In this case the representation of the geometry is the same as the graph associated with the geometry. What are the four parameters for this graph? Answer $(4,2,0,2)$ 


\section{Lesson 3: What are Strongly Regular Graphs?}

\section{Example 1}

These are two strongly regular graphs.
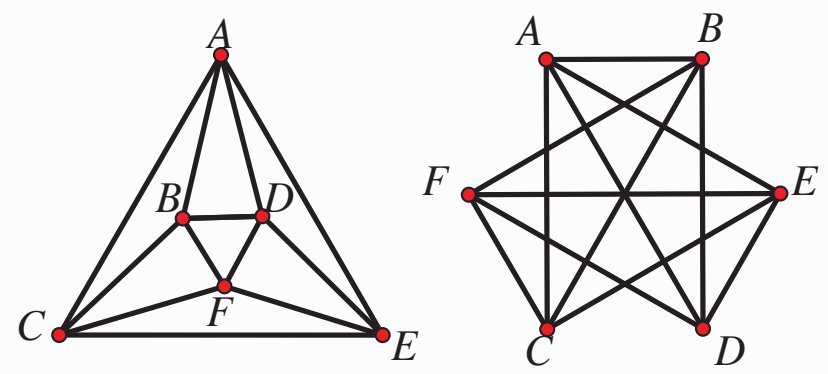

(a) What are the values of $v, k, \lambda$, and $\mu$ for each graph?

(b) Look the graph on the left and think about the geometry that might be associated with it. Suppose there are three points on each line of the geometry and $A B C$ is a line. What are the other lines in the geometry?

\section{Example 2}

Every partial geometry can be described by three parameters; $s, t$, and $\alpha$ (alpha). There are $s+1$ points on each line. There are $\alpha$ points on each line that are collinear with any point not on the line. This is written as $p g(s, t, \alpha)$.

(a) What are the values of $s, t$, and $\alpha$ for each geometry pictured here? 

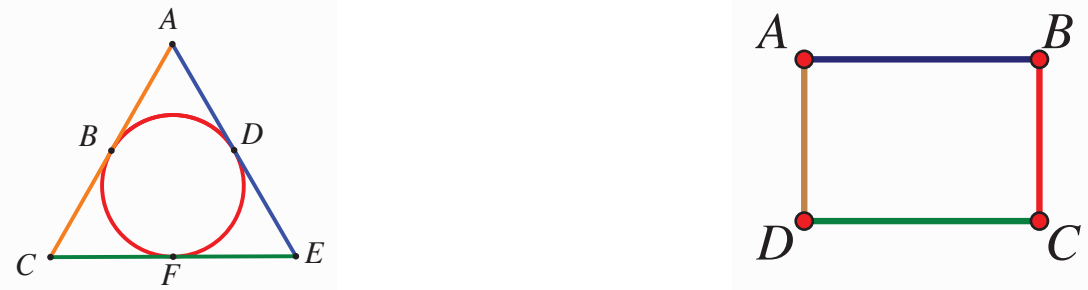

(b) The second geometry is also a strongly regular graph. What are the four parameters for this graph?

\section{In your group}

1. Decide if each graph below is strongly regular. If it is, identify the parameters $v, k, \lambda$, and $\mu$.
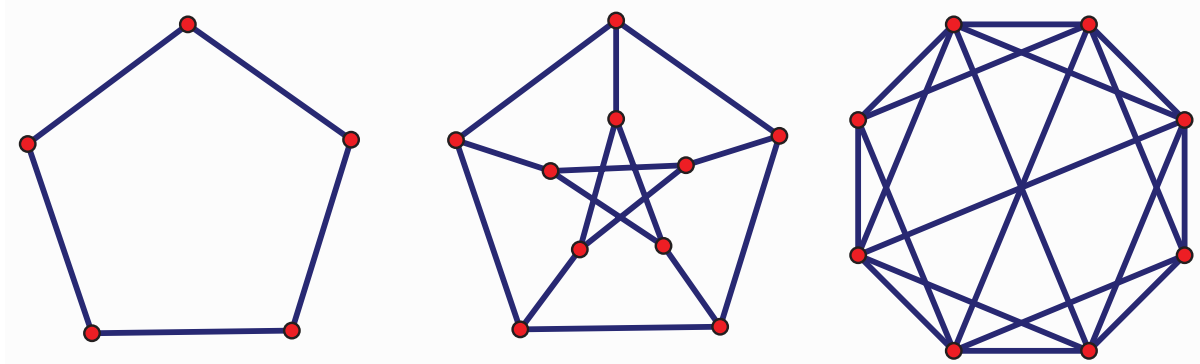
2. Consider each of the geometries pictured below.
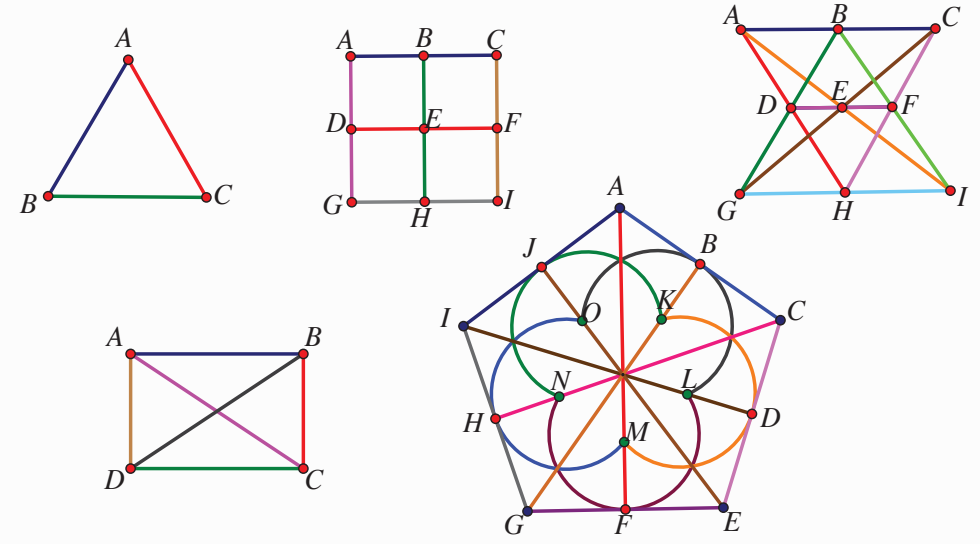

(a) Identify the values of $s, t$, and $\alpha$ for each partial geometry.

(b) Find $v, k, \lambda$, and $\mu$ for the graph associated with each geometry. Record your results below.

\begin{tabular}{|l|l|l|l|l|l|l|}
\hline$s$ & $t$ & $\alpha$ & $v$ & $k$ & $\lambda$ & $\mu$ \\
\hline & & & & & & \\
\hline & & & & & & \\
\hline & & & & & & \\
\hline & & & & & & \\
\hline & & & & & & \\
\hline
\end{tabular}

(c) Look at the values in your table. How can you find the value of $k$ from the values of $s$ and $t$ ? How can you find the value of $\mu$ from the values of $t$ and $\alpha$ ? 


\section{Lesson 3 answers}

\section{In your group}

1. $\operatorname{srg}(5,2,0,1), \operatorname{srg}(10,2,0,1)$, not a strongly regular graph, a regular graph of degree five but with no single value for $\lambda$.

2. (a) $p g(1,1,2), p g(2,1,1), p g(2,2,2), p g(1,2,2), p g(2,2,1)$

(b)

\begin{tabular}{|c|c|c|c|c|c|c|}
\hline$s$ & $t$ & $\alpha$ & $v$ & $k$ & $\lambda$ & $\mu$ \\
\hline 1 & 1 & 2 & 3 & 2 & 1 & \\
\hline 2 & 1 & 1 & 9 & 4 & 1 & 2 \\
\hline 2 & 2 & 2 & 9 & 6 & 3 & 6 \\
\hline 1 & 2 & 2 & 4 & 3 & 2 & \\
\hline 2 & 2 & 1 & 15 & 6 & 1 & 3 \\
\hline
\end{tabular}

(c) $k=s(t+1), \mu=\alpha(t+1)$ 


\section{Lesson 4: Proving the Connections}

In the previous lesson students conjectured that $k=s(t+1)$ and $\mu=\alpha(t+1)$. In this lesson picture proofs are presented and students must explain the reasoning displayed, completing the proof. The formulas for $v$ and $\lambda$ are more complicated and students will be led through this reasoning. The major result of this proof is that any partial geometry has an associated strongly regular graph and we can determine the parameters of that graph from the parameters of the geometry. However, the converse is not always true. There are many strongly regular graphs that do not correspond to a partial geometry. In trying to determine if a set of parameters creates a partial geometry, one can find the associated graph parameters. The adjacency matrix of this graph must also meet specific conditions for the partial geometry to exist. This use of indirect proof means the techniques of graph theory and linear algebra can provide insight into geometry. Trying to prove the existence or nonexistence of a geometry directly can be much more difficult. While none of these more advanced techniques are discussed in the student materials, it is worth discussing the use of indirect proof. In addition, many questions about partial geometries remain unanswered and are an area of active mathematical research. While it may seem that this field has little practical application, it has recently been discovered that the principles and theorems about partial geometries apply to some molecular structures. ${ }^{1}$ Knowledge about partial geometries may provide insight in this field.

Introduce the lesson by reviewing the conjectures from the day before. Also review the definition of $\alpha$. A clear understanding of all of the parameters will make the proofs much easier. Let the students work and struggle together to write the proofs. Encourage them to use complete sentences and to read their work aloud, making sure everyone believes the explanation to be clear and complete.

\footnotetext{
${ }^{1}$ M.Klin, G.Tinhofer (editors). A special issue of the journal MATCH, "Algebraic Combinatorics in Mathematical Chemistry", v.40, 1999
} 


\section{What are the Connections?}

Theorem 20 The point graph of a $\mathrm{pg}(s, t, \alpha)$ is a strongly regular graph with parameters $(v, k, \lambda, \mu)=\left((s+1)\left(\frac{s t+\alpha}{\alpha}\right), s(t+1), s-1+t(\alpha-1), \alpha(t+1)\right)$.

You will prove this in four parts, starting with those that you worked with in lesson 3. The pictures shown provide outlines for the proofs of each part. In each case $p$ represents a point, $p_{1}$ is a point collinear with $p$, and $p_{2}$ is a point that is not collinear with $p$.

\section{Part 2}

$k=s(t+1)$. Use the picture below to help you prove this part of the theorem.

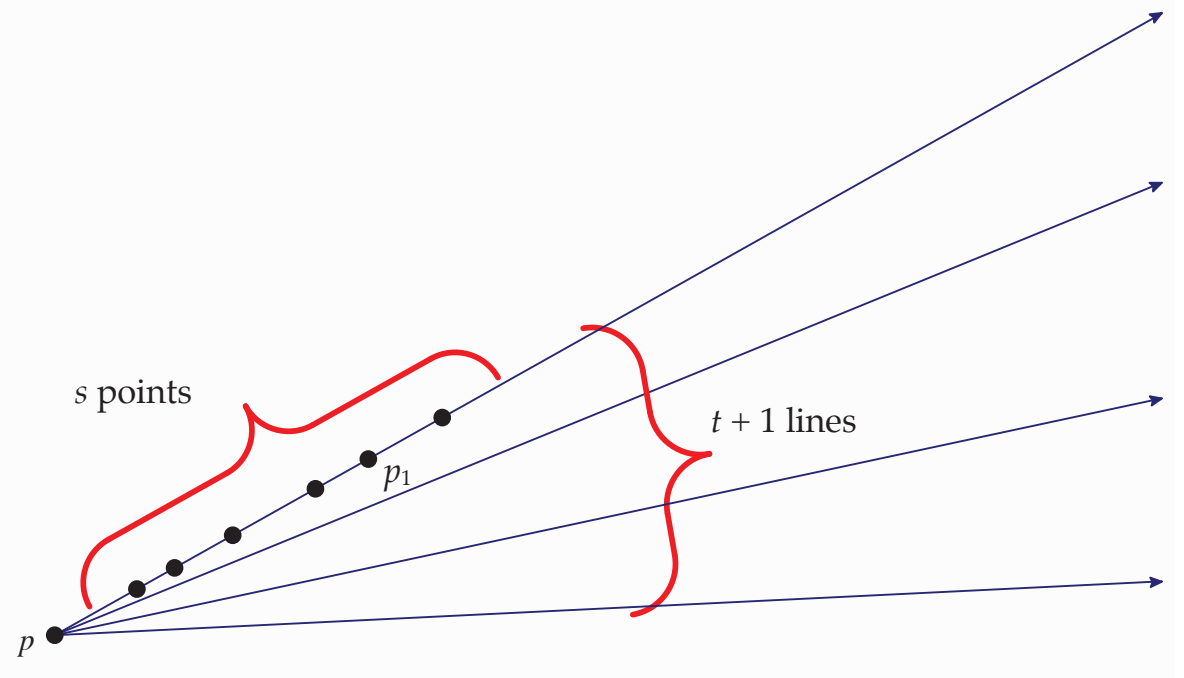




\section{Part 4}

$\mu=\alpha(t+1)$. Use the picture below to help you prove this part of the theorem.

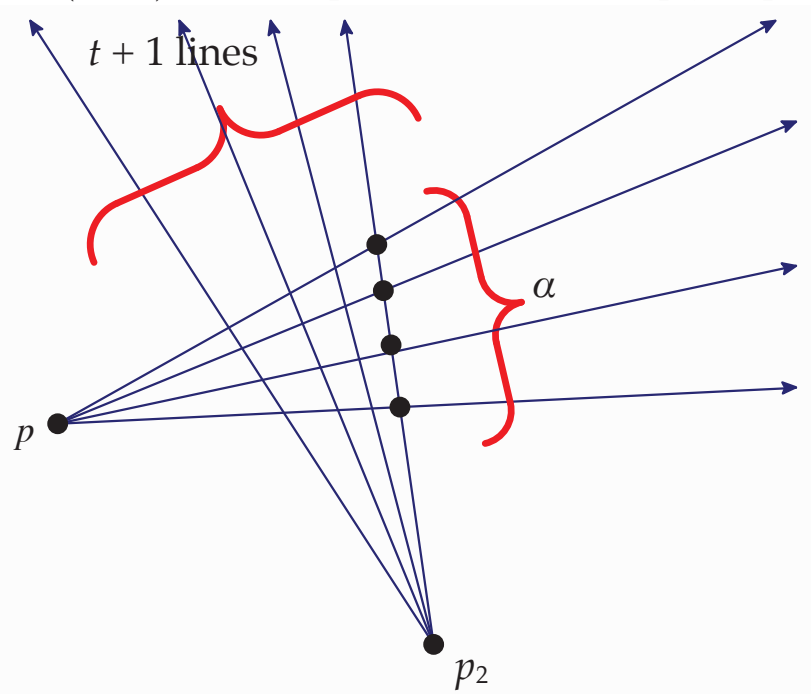




\section{Part 3}

$\lambda=s-1+t(\alpha-1)$. Use the picture below to help you prove this part of the theorem.

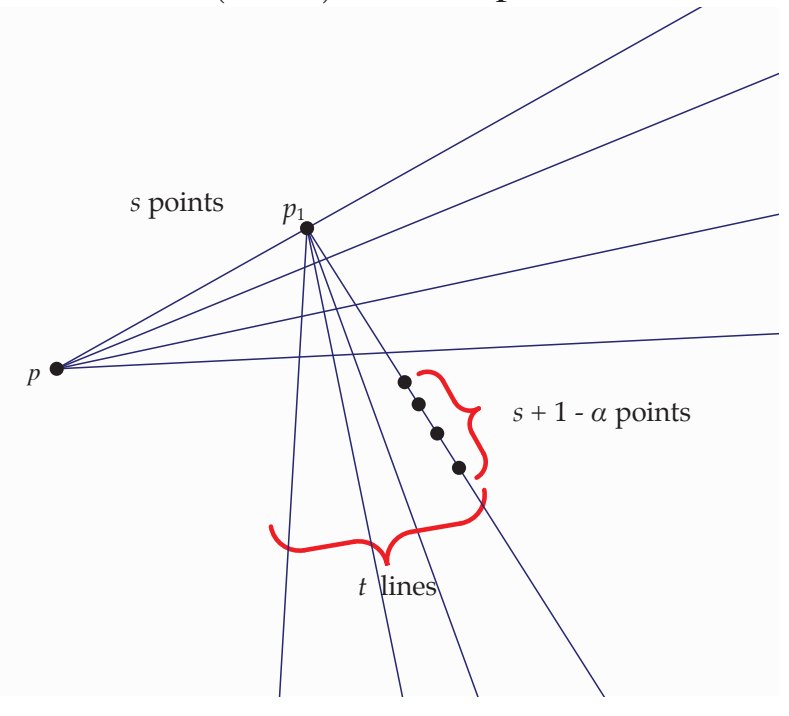




\section{Part 1:}

$v=(s+1)\left(\frac{s t+\alpha}{\alpha}\right)$. This is the most difficult part of the proof. To do this you will count the number of vertices in two different ways and then use algebra to actually solve for the total. As above, $p_{1}$ represents a point collinear with $p$, and $p_{2}$ represents a point not collinear with $p$. For any point $p$ you already know the number of possible points $p_{1}$. Call that number $P_{1} . P_{1}=$

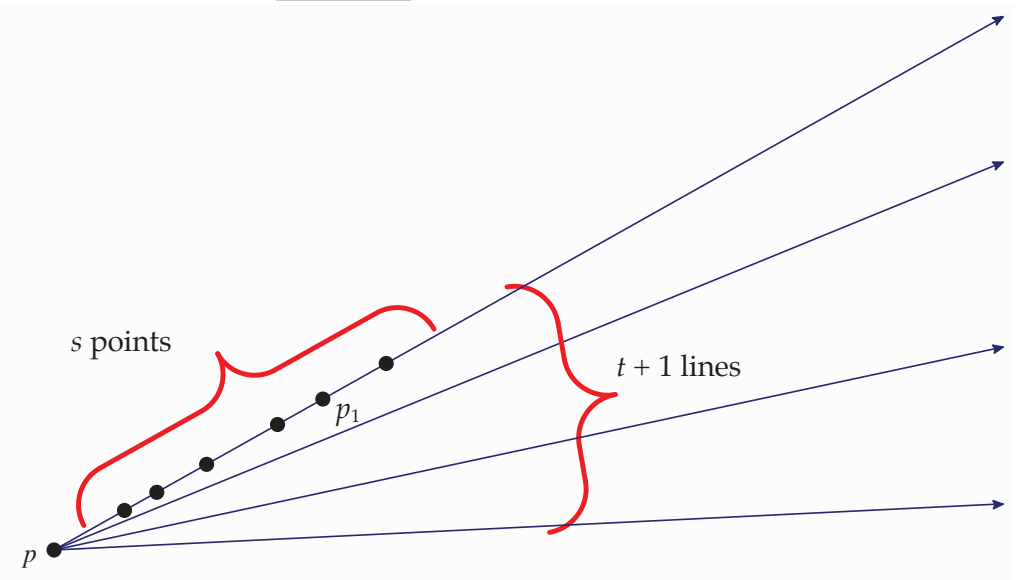

Use the picture below to find the number of $p_{2}$ points for each of the $p_{1}$ points.

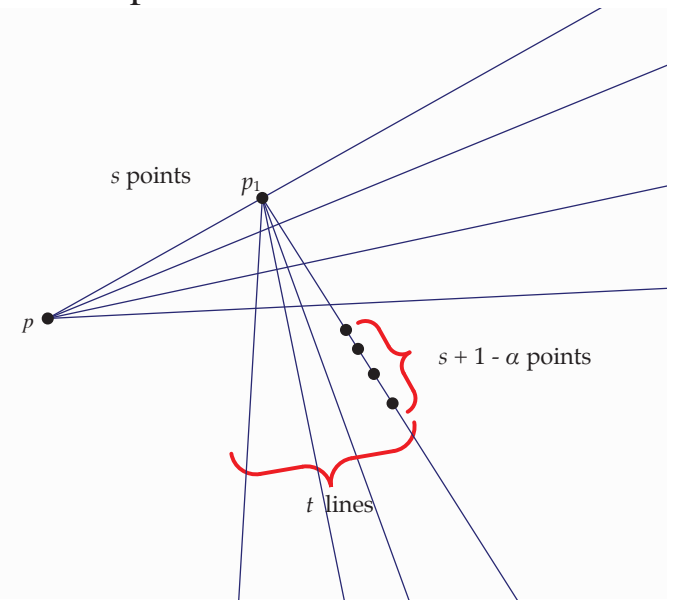

The total number of points of type $p_{2}$ points is $P_{2}$. The total number of pairs of points $\left(p_{1}, p_{2}\right)$ is $P_{1} \cdot P_{2}=$ 
You already know $\mu$, the number of points collinear with $p$ and any point $p_{2}$. So for any given $p_{2}$ you know $P_{1}=\mu=$

Now the number of pairs $\left(p_{1}, p_{2}\right)$ is $P_{1} \cdot P_{2}=\underline{P_{2}}$.

Set these two expressions for $P_{1} \cdot P_{2}$ equal to each other and solve for $P_{2}$.

Now the total number of points $P=1+P_{1}+P_{2}$. Here $P_{1}$ is the number of points collinear with $p$. You found this value at the beginning of this part. The 1 represents the chosen point $p$.

Use your expressions for $P_{1}$ and $P_{2}$ to find $P$. Simplify to get the desired result. 


\section{Lesson 4 answers}

\section{Part 2}

The number of vertices adjacent to a given vertex is the number of points collinear with a given point $p$. There are $t+1$ lines incident with each point and $s$ points on each line that are collinear with $p$. So there are $s(t+1)$ points collinear with a given point. Hence there are $s(t+1)$ vertices adjacent to each vertex.

\section{Part 4}

The number of vertices commonly adjacent to two nonadjacent vertices is the same as the number of points collinear with two noncollinear points. Let $p$ and $p_{2}$ be two noncollinear points. There are $t+1$ lines through $p_{2}$. None of them are incident with $p$. On each one there are $\alpha$ points that are collinear with $p$. So there are a total of $\alpha(t+1)$ points that are collinear with $p$ and $p_{2}$ and thus there are $\alpha(t+1)$ vertices that are commonly adjacent to two nonadjacent vertices.

\section{Part 3}

The number of vertices commonly adjacent to 2 adjacent vertices is the same as the number of points that are collinear with two given collinear points $p$ and $p_{1}$. Since each line contains $s+1$ points, there are $s-1$ more points on the line containing $p$ and $p_{1}$. There are $t$ lines through $p_{1}$, omitting the line containing $p$ and $p_{1}$. Each of these lines contains $\alpha$ points that are collinear with $p$. However $p_{1}$ is on each of these lines. So there are $\alpha-1$ points on each of the $t$ lines that are collinear to the two given collinear points. This makes a total of $s-1+t(\alpha-1)$ vertices commonly adjacent to 2 adjacent vertices.

\section{Part 1}

$P_{1}=s(t+1)$ from Part 2 .

For each of these $P_{1}$ points there are $t$ lines that are not incident with $p$. Each of these lines has $\alpha$ points that are collinear with $p$ and $s+1-\alpha$ points that are not. So there are $s+1-\alpha$ points, $p_{2}$, on each of these $t$ lines. Because two lines intersect in at most one point, none of these points is double counted. Thus for each $p_{1}$ there are $t(s+1-\alpha)$ points $p_{2}$. 
The total number of pairs of points $\left(p_{1}, p_{2}\right)$ is $s(t+1) t(s+1-\alpha)$.

For the second counting method, there are $P_{1}=\alpha(t+1)$ points collinear with $p$ and $p_{2}$. So the number of pairs $\left(p_{1}, p_{2}\right)$ is $P_{1} \cdot P_{2}=P_{2} \cdot \alpha(t+1)$.

Equating the two counts and solving for $P_{2}$ we get

$$
\begin{aligned}
& s(t+1) \cdot(s+1-\alpha)=P_{1} \cdot \alpha(t+1) \\
& P_{2}=\frac{s(t+1) \cdot t(s+1-\alpha)}{\alpha(t+1)}=\frac{s t(s+1-\alpha)}{\alpha}
\end{aligned}
$$

So the total number of points is

$$
\begin{aligned}
P & =1+P_{1}+P_{2} \\
& =1+s(t+1)+\frac{s t(s+1-\alpha)}{\alpha} \\
& =\frac{\alpha+s t \alpha+s \alpha+s^{2} t+s t-s t \alpha}{\alpha} \\
& =\frac{\alpha+s \alpha+s^{2} t+s t}{\alpha} \\
& =\frac{\alpha(s+1)+s t(s+1)}{\alpha} \\
& =\frac{(s+1)(\alpha+s t)}{\alpha} \\
& =(s+1)\left(\frac{s t+\alpha}{\alpha}\right)
\end{aligned}
$$

